Florida International University FIU Digital Commons

$11-4-2015$

\title{
Exploring How Transformational Experiences of Faculty Participating in Global Learning Workshops Inform Practice
}

Eduardo Hernandez

Florida International University, ehern003@fiu.edu

DOI: 10.25148 /etd.FIDC000180

Follow this and additional works at: https:// digitalcommons.fiu.edu/etd

Part of the Curriculum and Instruction Commons, Higher Education and Teaching Commons, and the Humane Education Commons

\section{Recommended Citation}

Hernandez, Eduardo, "Exploring How Transformational Experiences of Faculty Participating in Global Learning Workshops Inform Practice" (2015). FIU Electronic Theses and Dissertations. 2282.

https://digitalcommons.fiu.edu/etd/2282 


\section{FLORIDA INTERNATIONAL UNIVERSITY}

Miami, Florida

\section{EXPLORING HOW TRANSFORMATIONAL EXPERIENCES OF FACULTY \\ PARTICIPATING \\ IN GLOBAL LEARNING WORKSHOPS INFORM PRACTICE}

A dissertation submitted in partial fulfillment of the

requirements for the degree of

DOCTOR OF EDUCATION

in

CURRICULUM AND INSTRUCTION

by

Eduardo Hernandez

2015 
To: Dean Delia C. Garcia

College of Education

This dissertation proposal, written by Eduardo Hernandez and titled Exploring How Transformational Experiences of Faculty Participating in Global Learning Workshops Inform Practice, having been approved in respect to style and intellectual content, is referred to you for judgment.

We have read this dissertation proposal and recommend that it be approved.

$\begin{array}{r}\text { Tonette S. Rocco } \\ \hline \text { Joan T. Wynne } \\ \hline \text { Sarah A. Mathews } \\ \hline \text { Hilary C. Landorf, Major Professor }\end{array}$

Date of Defense: November 4, 2015

The dissertation proposal of Eduardo Hernandez is approved.

Dean Delia C. Garcia College of Education

Dean Lakshmi N. Reddi University Graduate School

Florida International University, 2015 
(C) Copyright 2015 by Eduardo Hernandez

All rights reserved. 


\section{DEDICATION}

This dissertation is dedicated to my family. I am deeply appreciative for the life, relationships, values, freedoms, and opportunities that have helped shape my journey. I also dedicate this dissertation to everyone whose support has helped to make this dissertation happen. Finally, I dedicate this dissertation to everyone who seeks to transform their lives and the lives of others. 


\section{ACKNOWLEDGMENTS}

I appreciate the help of everyone who has helped contribute in any way to this dissertation. Any level of success I have had at any point as a graduate student, I owe directly and for the most part to the support, dedication, guidance, and inspiration that my major professor, Dr. Hilary C. Landorf has offered. Dr. Landorf is a brilliant and positive force for change. I deeply appreciate the blunt and direct feedback of Dr. Tonette S. Rocco. I have learned a lot from Dr. Rocco directly and also from watching her interact with other students in the past and reading her writings on social justice issues. Dr. Sarah A. Mathews has been insightful throughout the dissertation process. I have also admired the activism, positive energy, and discourse that Dr. Joanne T. Wynne has brought to FIU. I would also like to acknowledge the participation of Dr. Maria Fernandez as a past committee member during a crucial time in my academic career.

Outside of the members of my committee, Dr. Linda Bliss and Dr. Isadore Newman have been a major influence on my work. Dr. Bliss and Dr. Newman have always been present in some way during the dissertation process no matter what direction it took.

I would like to also acknowledge the importance of two other people in particular who directly and indirectly helped in the construction of this dissertation. Dr. Stephanie Doscher was an important sounding post for ideas, a remarkable user of language, and an overall inspiration. Dr. Masha Plahotnik is inspiring as a role model. She helped make my scholarly writing outside the dissertation better through constructive criticism and this in turn, I believe has helped my dissertation. I also find her own work and use of scholarly language to be a major influence on my work. I also would like to thank Caprila 
Almeida and the staff at the Office of Graduate Studies for their hard work. There are other people that I met at the university that were helpful in my journey that I would like to thank but they are too many to name. I hope you know that you are all greatly appreciated. Finally, I would like to thank Dr. Jack Mezirow who I never met but whose work this dissertation is based on. 


\begin{abstract}
OF THE DISSERTATION
EXPLORING HOW TRANSFORMATIONAL EXPERIENCES OF FACULTY

PARTICIPATING IN GLOBAL LEARNING WORKSHOPS INFORM PRACTICE

by
\end{abstract}

Eduardo Hernandez

\author{
Florida International University, 2015. \\ Professor Hilary C. Landorf, Major Professor
}

This case study addresses gaps in the global learning, transformational learning, and professional development literature. Research is lacking on the questions of if and how university faculty members view global learning curricula as transformational learning as a result of professional development and how this transformational learning applies to their teaching of global learning curricula. This study's purpose is to explore whether university faculty members who have attended global learning professional development workshops perceive global learning as transformational learning and if they do, how they see global learning as transformational, and how this transformational learning informs their teaching.

Research questions were answered by using a survey and interview guide developed by the researcher. All faculty members at FIU who have attended a global learning professional development workshop were invited to take the survey to identify faculty members who have had a transformational learning experience related to global learning. Thematic analysis of the survey and interview questions helped describe how faculty members perceived global learning as transformational learning and how faculty 
members applied this transformational learning to their teaching of global learning curricula.

The study found that many university faculty members who have attended a global learning professional development workshop report a resulting transformational learning experience. These university faculty members perceive global learning as individual and collective opportunities and challenges. They apply this transformation to their teaching of global learning curricula through conceptual and individual instructional changes that facilitate and inform systematic awareness and systematic change.

This study is significant because how a global learning curricula is viewed by a faculty member affects how it manifests in the curricula, how it is taught, and what students glean from it. How FIU explores this topic could be adopted by other institutions. This study also builds on the work of Dr. Jack Mezirow in transformational learning. 


\section{TABLE OF CONTENTS}

CHAPTER

PAGE

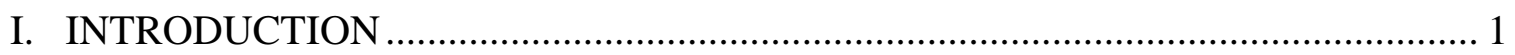

Background of the Problem .......................................................................... 1

Problem Statement ....................................................................................... 6

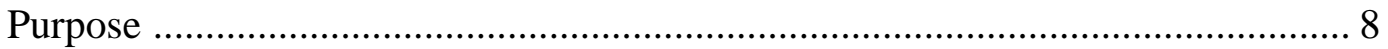

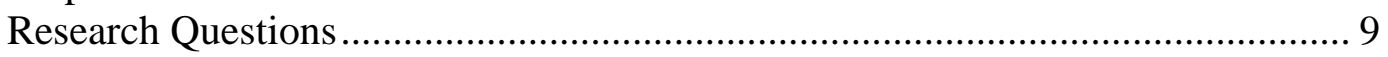

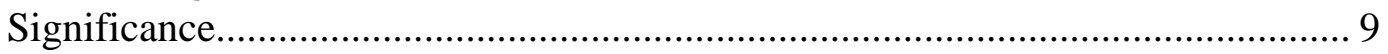

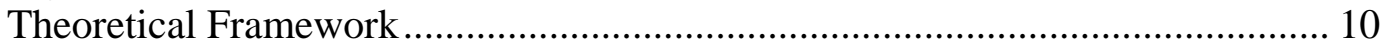

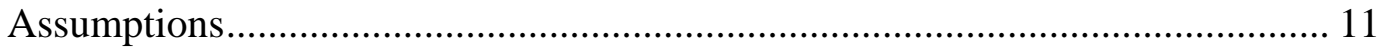

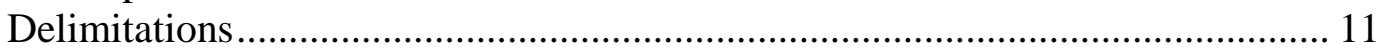

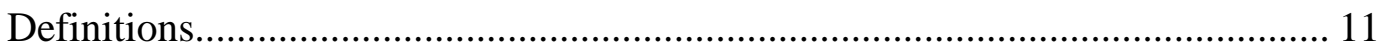

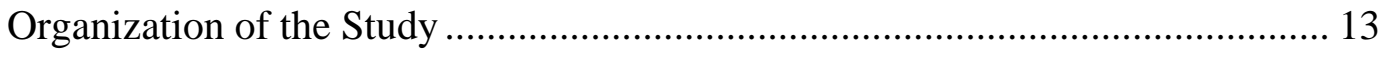

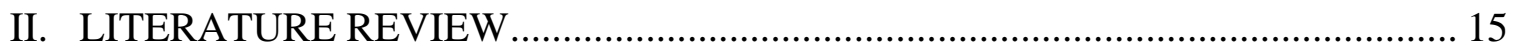

Organization of the Chapter............................................................................. 15

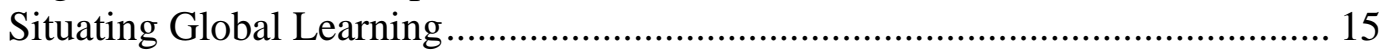

The Importance of Faculty.............................................................................. 22

Importance of Professional Development.......................................................... 26

Transformational Learning ............................................................................... 37

Literature on Faculty and Global Learning............................................................ 44

Literature on Transformational Learning and Global Learning ............................. 46

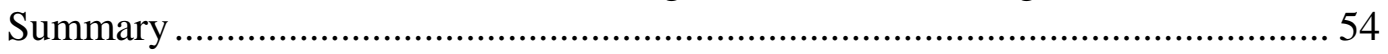

Overview of Succeeding Chapter …………………………….......................... 54

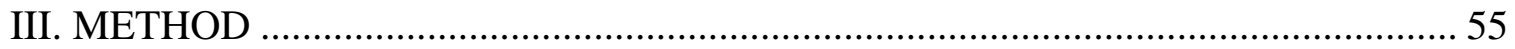

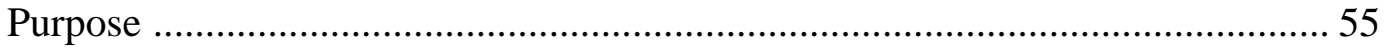

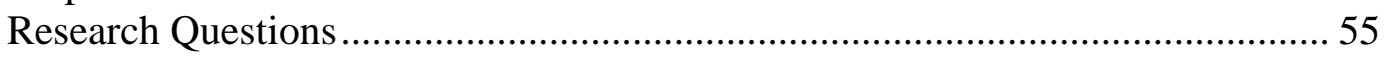

Research Design.............................................................................................. 56

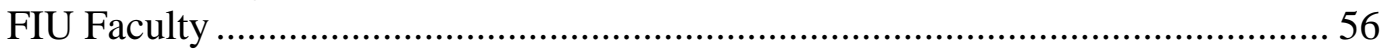

FIU’s Global Learning Professional Development Workshops ............................ 57

Population ........................................................................................................ 58

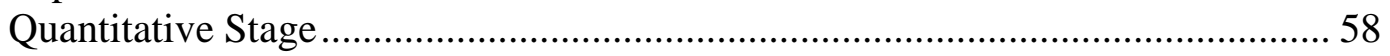

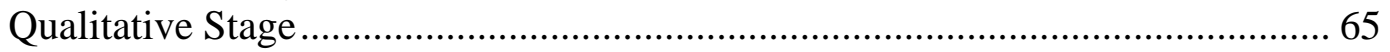

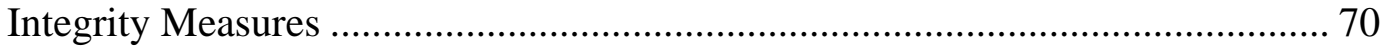

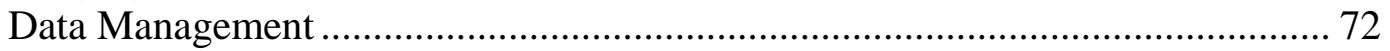

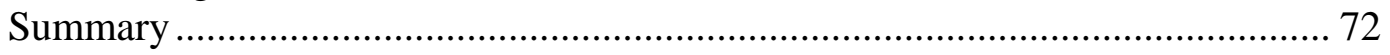

Overview of Succeeding Chapter .......................................................................... 72

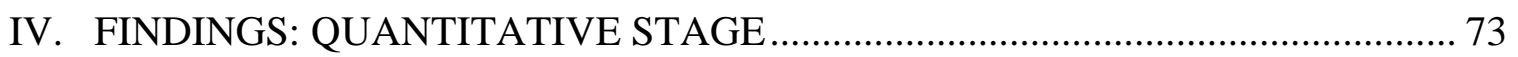

Quantitative Findings................................................................................... 74

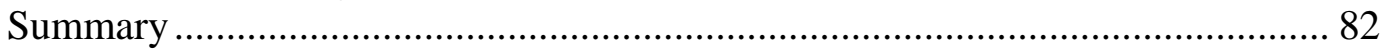


Overview of Succeeding Chapter ................................................................. 83

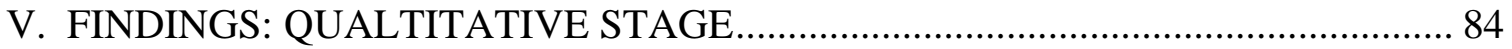

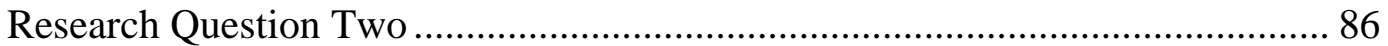

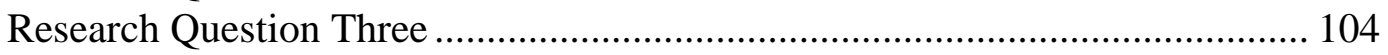

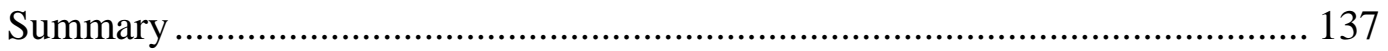

Overview of the Succeeding Chapter ............................................................. 138

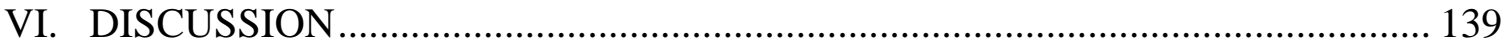

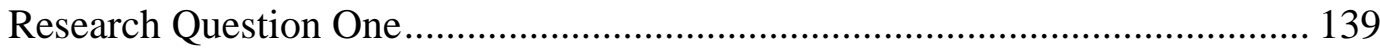

Research Question Two ................................................................................... 140

Research Question Three ........................................................................... 148

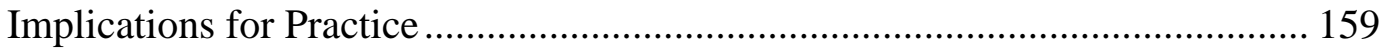

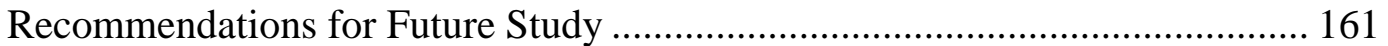

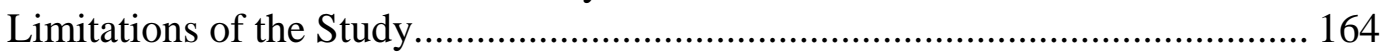

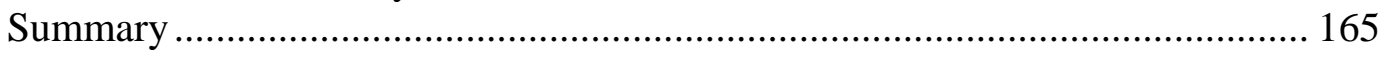

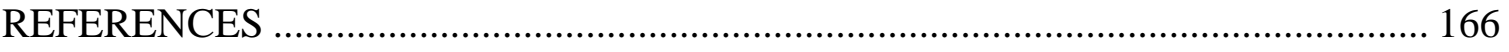

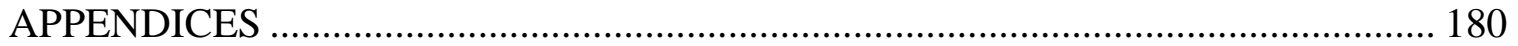

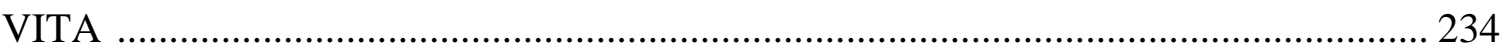




\section{LIST OF TABLES}

TABLE

PAGE

1. Transformational Learning Survey Results......................................73

2. Ethnicity of Faculty Members as Reported in Surveys..............................75

3. Race of Faculty Members as Reported in Surveys..................................76

4. FIU School or College of Faculty Members as Reported in Surveys...................77

5. Number of Global Learning Courses Taught by Faculty Members as Reported in

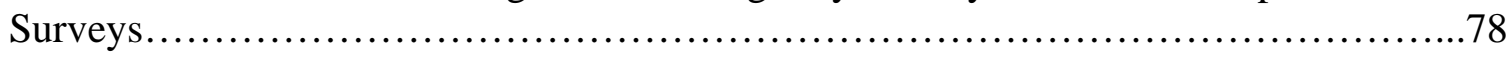

6. How Often Faculty Members Teach a Global Learning Class as Reported in

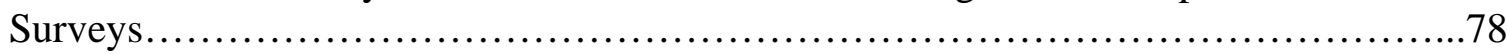

7. How Long Faculty Members Have Taught Global Learning Courses as Reported in

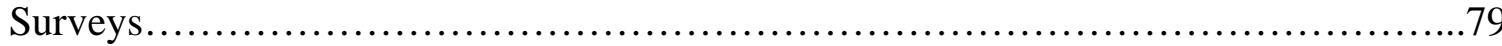

8. Number of Responses to Question/prompt(s) Two through 21 as Reported in the

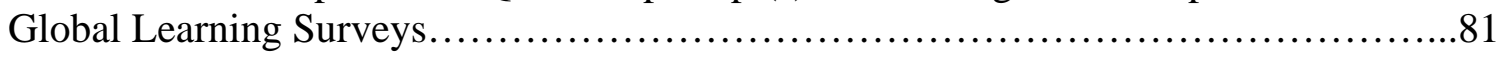

9. Major themes and subthemes in response to research question two.......................87

10. Major Themes and subthemes in response to research question three ................105 


\section{CHAPTER I \\ INTRODUCTION}

This dissertation outlines a case study that was undertaken to understand university faculty's views on global learning as transformational learning. The subject of this case study was the global learning workshops. Chapter 1 discusses the background of the problem, the problem statement, the purpose of the study, the research questions, the theoretical framework, assumptions, significance, delimitations and operational definitions. Chapter 1 ends with an overview of succeeding chapters.

\section{Background of the Problem}

Florida International University (FIU) opened its doors in 1972 with the goals of promoting teaching, service, and greater international understanding (Florida Department of State, 1976). For Charles Perry, the founding president of FIU, the notion of being an international institution meant promoting students' development of an awareness of the interconnectedness of global problems such as pollution, urbanization, and population growth and the skills to solve these problems in the context of their own lives (Florida International University, 1974). Since its founding, the university has grown tremendously in the scope of its activities. FIU has over 175 bachelors, masters, and doctoral degree programs offered through its colleges and schools. FIU has gone from serving a student body of just over 5,000 students to accommodating over 50,000 students (Florida International University, 2012-2013). The majority of these students are from ethnic minority groups (primarily Hispanic), are first generation university students, first or second generation U.S. citizens, live at home, and work while attending school fulltime. The Carnegie Foundation for the Advancement of Teaching ranks FIU as a 
Research University in the High Research Activity Category. FIU has 10 colleges and schools that enroll undergraduate students. The university also has Colleges of Law and Medicine (Florida International University, 2014).

In 2007, a curriculum program review revealed an “internationalization gap” at the university (Florida International University, 2010). In a review of many of the existing undergraduate programs, the Office of Planning and Institutional Research found that internationalization was widely recognized as important in academic program mission statements and goals, but not widely reflected in the student learning outcomes in the curriculum (Landorf \& Doscher, 2013). A survey also was conducted amongst a wide array of FIU stakeholders including students, faculty, staff, alumni, and members of the community, with the aim of bolstering FIU's brand (Stamats, 2008). Analysis of the survey results revealed that while stakeholders were proud of the perceived cultural and national diversity of the student body and faculty, that diversity was largely absent in the curriculum. This was followed by the eventual establishment of a university-wide internationalization of the curriculum initiative as the centerpiece of its 2010 application for reaffirmation of accreditation from the Southern Association of Colleges and Schools (Florida International University, 2010). An internationalized curriculum is a "curriculum with an international orientation in content and/or form, aimed at preparing students for performing (professionally/socially) in an international and multicultural context and designed for domestic and/or foreign students” (Organization for Economic Co-operation and Development (OECD, 1996, p. 9).

Internationalization of the curriculum is part of the larger process of comprehensive internationalization. Comprehensive internationalization, as defined by 
American Council on Education's Center for Internationalization and Global Engagement (CIGE), is:

a strategic, coordinated process that seeks to align and integrate international policies, programs, and initiatives, and positions colleges and universities as more globally oriented and internationally connected. This process requires a clear commitment by top-level institutional leaders, meaningfully impacts the curriculum and a broad range of people, policies, and programs, and results in deep and ongoing incorporation of international perspectives and activities throughout the institution. (CIGE, 2012, p. 3)

Internationalization at a higher education institution can be deeply influenced by the history, resources, and stakeholders of an institution (Knight, 2004). Transformational change in an institution can be affected by how these individuals view their world (Henderson, 2012).

Once FIU's internationalization gap was identified, which led to the willingness and desire to institute large-scale curricula change, an interdepartmental committee and several subcommittees grappled with various questions related to an internationalized curricula, what FIU had to offer, and the needs of the students in a global context. This was soon followed by the establishment of an Office of Global Learning Initiatives (OGLI). The OGLI is responsible for providing leadership, research, professional support, and assessment for this large scale curricula reform (Florida International University, 2010).

Influenced by the human capability approach, the OGLI promoted a dialogic process to help FIU find overlapping consensus concerning student learning with a 
representative group of FIU stakeholders including students, academics, administrators, staff, alumni, parents, local businesses, and community members. This process helped the FIU community make a paradigm shift where conceptions of what is global and what is international were clarified and differentiated, where the community could understand that being global was not just about content but a way of being, and where consensus was reached to focus on global citizenship. Global citizenship was presented as an attitude that results from a combination of global awareness and a global perspective, or "the willingness of individuals to apply their knowledge of interrelated issues, trends, and systems and multi-perspective analytical skills to local, international, and intercultural problem solving” (Florida International University, 2010, p. 14). The FIU community decided that it needed to reform its curricula in order to develop the knowledge, skills, and attitudes of global citizenship in undergraduate students through a process of global learning (Florida International University, 2010).

There are various definitions of global learning. A common point for the development of most definitions is that global learning is education where the goal is the development of global citizenship (Elrod \& Hovland, 2011). Hovland, Senior Director of Academic Programs at NAFSA, and the former Senior Director of Global Learning and Curricular Change at the Association of American Colleges and Universities (AAC\&U), defined global learning as the process by which students are prepared to fulfill their civic responsibilities in a diverse and interconnected world (2006). Hilary Landorf, Director of the Office of Global Learning Initiatives at FIU, has defined global learning as the process of diverse people collaboratively analyzing and addressing complex problems that transcend borders (Landorf \& Doscher, 2015). Global learning differs from an 
internationalized curricula in the sense that recognizing problems and solutions do not just demand thinking and acting outside of one's nation or several nations but globally (ACE, 2008, Hovland, 2006; 2005; Musil, 2006). International education consultant Green (2006) relates global learning to internationalized curricula in her assertion that global learning is a process that reforms and informs education, a kind of learning, and also the activities and concepts involved in an internationalized curricula. In light of this view, the term global learning in this study is hereafter used synonymously with global learning curricula.

Globalization is a constantly changing configuration of networks, systems, and innovations in communication, technology, production, and supply delivery chains that are changing the boundaries, economies, power structures, agency, inequalities, and opportunities everyone faces in real time (Drucker, 1999). Global learning helps students address globalization by helping them become global citizens who can see, value, and participate in the solving of global problems. In the United States, issues arising out of destabilization and disruption due to globalization has spurred leaders to promote global learning to every undergraduate in American colleges and universities. FIU is a recognized early adopter and leader in the promotion of global learning in American higher education (Greene, 2012).

FIU specifically describes global learning as involving a process composed of active, team-based exploration of real-world problems and issues (Landorf \& Doscher, 2013). The reform process of the internationalization of the curricula at FIU eventually led to the launching of Global Learning for Global Citizenship in 2010. Global Learning for Global Citizenship is a university wide integrated curricular and co-curricular 
initiative whose purpose is to provide every FIU undergraduate with educational opportunities to achieve the knowledge, skills, and attitudes of global citizenship (Florida International University, 2010). The heart of the initiative is a requirement that all FIU undergraduate students take a minimum of two global learning courses prior to graduation: one interdisciplinary foundations course as part of the university core curriculum (UCC) sequence and a second discipline-specific global learning course in the context of their major program of study. All FIU global learning courses address three student learning outcomes. The student learning outcomes are global awareness, global perspective, and global engagement. Global awareness is "the knowledge of the interrelatedness of local, global, international, and intercultural issues, trends, and systems." Global perspective "is the ability to conduct a multi-perspective analysis of local, global, international, and intercultural problems.” Global engagement is “an attitude marked by a willingness to engage in local, global, international, and intercultural problem solving” (Florida International University, 2010, p. 23).

\section{Problem Statement}

Much of the research that has been conducted on global learning has been focused on students (Loveland, 2011; Gibson, Rimmington, \& LandwherBrown, 2008). This research focused on faculty perceptions of global learning. There is little research on faculty in relation to global learning curricula (Barbiero, 2008; Lenz \& Warner, 2011; Perry \& Mander, 2005; White, 2005) and none on how faculty perceive global learning curricula. The global learning research specifically about FIU has focused on the students in studies including the development of rubrics to assess their progress (Doscher, 2012), describing the development of student learning outcomes and related issues and activities 
(Landorf \& Doscher, 2012), and an exploration of the resources and capabilities of the institution to promote global learning to students (Landorf \& Pineda, 2012). At the center of the promotion of global learning, is not just the students but the educators also. Indepth research is absent on how faculty members perceive global learning curricula at FIU. Iuspa (2010) for example, as part of larger study on internationalization, focused on faculty attitudes on the benefits, support, and curricula of internationalization but not global learning curricula.

University faculty can have a conception or belief about teaching (Kember, 1997). A perspective on teaching is an interrelated set of beliefs, intentions, and actions linked to knowledge, learning, and the role of a teacher (Pratt, 1992). The practice of reflecting on one's teaching perspective demands that faculty members stand outside themselves to explore the assumptions that support what they teach and how they teach it (Brookfield, 1995). Having faculty members reflect on their teaching perspective is important because their perspective affects what is taught, how it is taught, and various other practices and activities in higher education (Cranston, 2001) and the work of the rest of the university community (Kember, Kwan, \& Ledesma, 2001). Research in Europe, Asia, and other countries (Eley, 2006) has focused on ascertaining teaching perspectives and categorizing them (Pratt, 2002). Many universities are using professional development as vehicles to promote reflection and dialogue about teaching perspectives (Hubbal, Collins, \& Pratt, 2006).

Another aspect of teaching is transformational learning which is when "learning is understood as the process of using a prior interpretation to construe a new or revised interpretation of the meaning of one's experience in order to guide future action” 
(Mezirow, 1996, p. 162). Of importance to transformational learning is the need for critical reflection on the perspectives one has (Henderson, 2002; Mezirow, 1991). Promoters of global learning argued that global learning transforms the way students view their world (Hovland, 2010). FIU has sought to make a major transformation not only in the way their students view the world, but in the way their faculty teach and view the curricula. FIU has developed highly structured professional development workshops to help faculty reflect and collaborate on the promotion and teaching of the global learning curricula and faculty development is a precursor to the teaching of a global learning curricula (Greene, 2012). For many university faculty members, global learning is a major challenge to the way they see the teaching of the content knowledge of their respective subject areas (Duerst-Lahti, 2005; Takacs, 2008). Critical reflection in professional development workshops could have led to incidences of transformational learning amongst university faculty members due to the teaching of global learning curricula. Previous research has focused on professional development, reflection, and teacher perspectives in higher education before (Eley, 2006) and using professional development for transformational learning (King, 2002). However research on professional development, faculty reflection, and transformational learning in relation to the teaching of global learning curricula by faculty is lacking. Research of this kind at FIU offers the opportunity to expand on the literature of global learning and professional development.

\section{Purpose}

The purpose of this dissertation was to expand on the literature on global learning and professional development by exploring whether university faculty members who 
have attended global learning professional development workshops perceive global learning as transformational learning, and if they do, how these faculty members see global learning as transformational, and how this transformational learning informs their teaching.

\section{Research Questions}

This study sought to answer these main research questions: Do university faculty members who have attended global learning professional development workshops report a transformational learning experience? How do university faculty members who have attended global learning professional development workshops perceive global learning curricula as transformational learning? How do university faculty members who have attended a global learning professional development workshop and report having had a transformational learning experience apply this transformation to their teaching?

\section{Significance}

This study is significant because how a global learning curriculum is viewed by a faculty member affects how it is taught, and what students get from the experience of being taught a global learning course. Critical reflection by faculty members on and during their practice helps faculty resolve issues in the curricula (Schon, 1983) and helps create a consonant relationship between what they intend to teach, what they actually teach, and what the students actually learn (Thornton, 1988). The way FIU explores this topic could be adopted by other institutions seeking to use global learning curricula. This study is also significant because it builds on the work of Mezirow $(1978,1991,2001)$ and King (1997, 1998, 2002, 2004). King (1997) expanded the literature concerning Mezirow’s $(1978,1991,2001)$ transformational learning theory by researching 
transformational learning in professional development and in higher education and the various learning activities that could have supported the transformation that adult learners went through.

\section{Theoretical Framework}

The framework for this study is Mezirow's (1978; 1991; 2001) theory of transformational learning. Mezirow (1978) developed the theory of transformational learning after conducting a qualitative study to explore the needs of women in the United States who were resuming employment or education after an extended period time out of the university or out of the workforce. The goal of his research was to understand the factors that helped or facilitated the women's progress in integrating themselves into school and work life. The study explored 12 reentry college programs with 83 women in four states. Further follow up studies were done through telephone and mail inquiry of several reentry programs nationwide.

The research showed that respondents had undergone a personal transformation after becoming critically aware of what they expected of themselves and what was expected of themselves by society (Mezirow, 1978). The idea of transformational learning is that a person could have a situation that gradually or quickly changes the way they view life. Reaching this transformation of perspective is done after several steps. An incident, for example, could cause a person to question themselves. A human being has a habitual set of expectations when it comes to things they see in their reality and these expectations are a kind of belief system. When the individual encounters something in their reality that challenges their prior knowledge and they reflect on it, they try to make sense of it. In that process, the individual may have to adjust or change their belief system 
and subsequently the actions they take in life. This change in knowledge, beliefs, and actions is transformational learning (Mezirow, 1978; 1991; 2001).

\section{Assumptions}

The following were assumptions the research held in this study:

1. Transformational learning is a phenomenon a university faculty member can experience, perceive and describe.

2. University faculty members can impart important knowledge and insights about global learning curricula.

\section{Delimitations}

This study was delimited to university faculty members at FIU who have been taught global learning curricula at a faculty development workshop as identified by FIU’s Office of Global Learning Initiatives.

\section{Definitions}

\section{Faculty}

Faculty is defined as "positions assigned the principal responsibility of teaching, research, and public service activities or for administrative responsibility for functions directly related to the academic mission” (Florida International University, 2014, p. 6.).

\section{Global Awareness}

Knowledge of the interrelatedness of local, global, international, and intercultural issues, trends, and systems (Florida International University, 2010, p. 23).

\section{Global Citizenship}

An attitude that results from a combination of global awareness and a global perspective, or "the willingness of individuals to apply their knowledge of interrelated 
issues, trends, and systems and multi-perspective analytical skills to local, international, and intercultural problem solving”(Florida International University, 2010, p.14).

\section{Global Education}

Global Education was outlined by Merryfield (1997) as the study of human beliefs and values, global systems, global issues and problems, global history, cross cultural understanding/interaction, awareness of human choices, the development of analytical and evaluative skills, and strategies for participation and involvement.

\section{Global Engagement}

An attitude marked by a willingness to engage in local, global, international, and intercultural problem solving (Florida International University, 2010).

\section{Global Learning}

Florida International University has defined global learning as a process composed of active, team-based exploration of real-world problems and issues (Florida International University, 2010).

\section{Global Learning for Global Citizenship}

This is a university-wide initiative developed and implemented by FIU. The purpose of this initiative is to provide every FIU undergraduate with educational opportunities to achieve the knowledge, skills, and attitudes of global citizenship by providing global learning opportunities across the curriculum and co-curriculum (Florida International University, 2010).

\section{Global Perspective}

This is the ability to conduct a multi-perspective analysis of local, global, international, and intercultural problems (Florida International University, 2010, p. 23). 


\section{Internationalized Curriculum}

"A curriculum with an international orientation in content and/or form, aimed at preparing students for performing (professionally/socially) in an international and multicultural context and designed for domestic and/or foreign students” (OECD, 1996, p. 9).

\section{National Citizenship}

National citizenship is defined as a set of rights and responsibilities granted by the nation-state (Steenburgen, 1994).

\section{Student Learning Outcomes}

A measurable outcome focused on students' knowledge, skills, or attitudes after completing a degree program with the graduating student as the unit of analysis (Doscher, 2012, p. 25).

\section{Transformational Learning}

Transformational learning theory is when "learning is understood as the process of using a prior interpretation to construe a new or revised interpretation of the meaning of one’s experience in order to guide future action” (Mezirow, 1996, p. 162).

\section{Organization of the Study}

This dissertation consists of six chapters. Chapter 1 has provided an introduction to the research. Chapter 2 provides a literature review. That chapter includes an exploration of faculty and the concepts of global learning, reflection in professional development, and transformational learning. Chapter 3 outlines the research design, sample, data collection and data analysis of the dissertation. Chapter 4 contains the 
quantitative findings of the dissertation. Chapter 5 contains the qualitative findings of the dissertation. Chapter 6 provides a discussion of the findings, includes recommendations for practice and future research, and concludes the study. 


\section{CHAPTER II}

\section{LITERATURE REVIEW}

\section{Organization of the Chapter}

This literature review is organized in two sections. In the first section, the major concepts of this dissertation are explored. First global learning is situated within the context of internationalization and comprehensive internationalization. Then the importance of faculty is explored. This is followed by an examination of the importance of professional development. Transformational learning is then explored. Since this dissertation is focused on faculty and global learning and transformation, the second section of this chapter consists of a literature review of research on faculty and global learning and a literature review on research on transformation learning and global learning.

\section{Situating Global Learning}

This introduction situates global learning in the context of internationalization and comprehensive internationalization. Research that firmly situates global learning within the larger context of internationalization and comprehensive internationalization (CIGE, 2012; Green, 2003; Olson, Green, \& Hill, 2005; 2006), shows the significance of global learning as part of a larger deliberate process to reform education in the United States. Internationalization "at the national/sector/institutional levels is the process of integrating an international, intercultural, or global dimension into the purpose, functions, or delivery of higher education at the institutional and national levels” (Knight, 2008, p. 21). Forms of internationalization have been present since ancient times (de Wit, 1998; Knight \& de Wit; 1995; Merkx \& de Wit, 2013). Various goals and objectives characterize 
contemporary motivations to internationalize. First, the increasing frequency of crosscultural contacts and relationships is promoting the need for expanding cross-cultural knowledge and understanding. In a global system of education, Higher education institutions also aim to raise their stature and value in or through teaching and research via internationalization. Internationalization is also seen as an enhancement to national and global security. Internationalization is also viewed as a means to improve labor forces and the ability of local economic systems to compete or survive with larger global systems. Finally, internationalization helps individual students by enhancing or developing the knowledge, skills, and attributes they need in order to make them effective work force members and citizens (Hudzik, 2011).

Funded by the Ford Foundation, the American Council on Education (ACE) conducted several investigations starting in 1998 into the state of internationalization in the United States and mapped the promising practices colleges and universities had made toward improving undergraduate education. With support from the Carnegie Corporation and W.K. Kellogg Foundation, ACE created several projects to improve Internationalization. ACE built on the work of the Association of International Education Administrators and other researchers and took inspiration from work done in Europe on internationalization by institutions such as the Institute for Management in Higher Education and the European University Association. The end result is a heavy push to reform higher education. Internationalization would eventually be replaced with the notion of comprehensive internationalization (Green, 2003).

As the leading association for U.S. colleges and universities, ACE has played a central role in supporting campus internationalization efforts through their initiatives. The 
ACE Global Learning project resulted in several publications on comprehensive internationalization which have been the cornerstone of such efforts in U.S. higher education (Olson, Green, \& Hill, 2005; 2006). Comprehensive internationalization, as defined by ACE’s Center for Internationalization and Global Engagement (CIGE), is “a strategic, coordinated process that seeks to align and integrate international policies, programs, and initiatives, and positions colleges and universities as more globally oriented and internationally connected. This process requires a clear commitment by toplevel institutional leaders, meaningfully impacts the curriculum and a broad range of people, policies, and programs, and results in deep and ongoing incorporation of international perspectives and activities throughout the institution” (CIGE, 2012, p. 3).

CIGE's Model for comprehensive internationalization encompasses the following six interconnected target areas for initiatives, policies, and programs: The first is an articulated institutional commitment. The second is administrative structure and staffing. The third are curriculum, co-curriculum, and learning outcomes. The fourth is faculty policies and practices. The fifth target area is the promotion of student mobility and the sixth target area is the promotion of collaboration and partnerships (CIGE, 2012). Global learning is a process that can be used in the internationalization of the curriculum and cocurriculum and learning outcomes. Global learning informs other areas of comprehensive internationalization, and is informed by these areas as well.

Loveland (2011) argued that global learning is defined in part by how it differs from international education. The concept of international education literally means between nations. International education represents a way of thinking about the world that was dominant a half century ago when our leaders focused on power and cultural 
relations between different nations and across the Soviet/Western power axes. The AAC\&U has been promoting the term global learning to emphasize the interconnectedness of people and issues in the global community, and also to emphasize that the United States is very much part of a global community. Global learners need to see themselves as part of a larger community—not just as a discrete nation that has formal interaction with others. The term "global learning” supports the need for multidimensional approaches to the big questions that students face as part of a planet. These questions can be of climate, sustainability, energy, water, food, poverty, and/or disease to name a few (Loveland, 2011). Global learning is a process that differs from traditional internationalization by focusing on systems and phenomenon that are global or planetary in nature (ACE, 2008).

The need to look at issues globally manifested itself in recent decades through many other influential voices in the popular culture outside of the university context. This section briefly goes over this global view of problems. Carson (1962) explored pollution as a global issue. Fuller (1969) explored the environment. Erlich (1968) explored population growth on a planetary scale. McLuhan (1964) explored the planetary impact of changes in communications technology. The need to look at issues globally also manifested itself in other strains of education beyond the university too. Several thinkers decided to stress the importance of teaching global issues to K-12 students. Hanvey (1976) developed a model for developing a global awareness that promoted the idea students become conscious of their perspectives, conscious of the perspectives of others, the global systems they confront, and the decisions they make as humans. In social studies, the concept of global education began to emerge from various settings such as 
international relations, cultural studies, environmental study, and economics (Kirkwood, 2001; Lamy, 1990). The concept of global education is tied to the idea that students will be facing a new world order where students have to attain a global focus to understand and solve problems and that K-12 teachers need to be prepared to teach them accordingly (Kirkwood, 2001). These studies are important to this dissertation because they show that other forces from the ground up beyond the university are promoting the significance and need for global learning.

By 2003, much of the terminology for global citizenship, global learning, internationalization, education for sustainability, and so on was used interchangeably or treated as though they were different but closely related by many researchers (Hicks, 2003). Definitions of global learning are evolving although the AACU claims that there is more and more agreement about outcomes, goals, practices and methods and this creates a kind of general framework for global learning (Elrod \& Hovland, 2011). Global learning according to AACU for example "is the process by which students are prepared to fulfill their civic responsibilities in a diverse and interconnected world” (Hovland, 2006). The American Council on Education (ACE) defines global learning as "the knowledge, skills, and attitudes that students acquire through a variety of experiences that enable them to understand world cultures and events; analyze global systems; appreciate cultural differences; and apply this knowledge and appreciation to their lives as citizens and workers” (ACE, 2008).

In the shared futures initiative, the AACU uses the term "global” rather than "international." Research suggests that the term "international” is most often associated in the academic community with study-abroad programs, international students on 
campus, the study of foreign languages, and international affairs. The term "global," while still evolving in use, most often applies to dynamic processes and the flows of people, cultures, labor and capital, diseases, and resources across and between borders. In these terms, a "global learning” framework seems to offer more intellectual and curricular space in which students and faculty can explore the critical relationship between U.S. diversity and its global contexts (Hovland, 2005c). Global learning as a term has begun to appear more and more in the mission statements of universities (Hovland, 2005b).

Gibson, Rimmington, and LandwherBrown (2008) describe global learning as a student-centered activity in which learners of different cultures use technology to improve their global perspectives while remaining in their home countries to develop the knowledge, attitudes, and skills necessary for world citizenship. Global learning can be defined in part as a network and as a way to fight for justice, equality, and democracy through the promotion of intercultural interaction, collaborative work, and the promotion of critical inquiry (Cummings \& Sayer, 1997). Even simple forms of global learning such as discussing a foreign film with someone from another culture can help the student become a cosmopolitan who can care for people beyond the borders of their state or culture. These global citizens can help solve global problems like pollution with minimal effort. Raising awareness of problems by simply discussing it is an example (Appiah, 2010). Global learning has expanded as a concept and as a promoter of institutions spurred by major events like September $11^{\text {th. }}$. When the AACU originally queried its members about definitions of global learning, responses were forward to departments dealing with international education and study abroad. Currently, various global learning 
initiative and programs exists where there was none a decade ago (Elrod \& Hovland, 2011).

Hovland (2009) argued that while definitions of global learning are continuing to evolve but that global learning is the responsibility of everyone. Hovland highlights how past AAC\&U initiatives that dealt with the querying of business leaders, the conducting of focus groups, and the taking of national surveys in order to determine what is needed in our present world for employability and in education demonstrated that what seemed to be lacking in students was an understanding of global issues and global knowledge. These discussions led to the exploration of student learning outcomes. Attempts to define global learning were complicated but consensus built around student learning outcomes helped spur the discussion on global learning. Many institutions and leaders came up with their own definitions of global learning (Hovland, 2009). In FIU for example, university faculty were part of the stakeholders queried in that institution to define the concept of global learning, the process of global learning, and the goals and outcomes of global learning curricula (Landorf \& Doscher, 2012).

More and more of these academic institutions, are defining global learning as a vehicle for integrating multidisciplinary perspectives and weaving together existing commitments to explore diversity, build capacity for civic engagement, and to make students take responsibility for common global problems. Hovland stated that ...global learning is knowledge focused by engagement with big questions; skills practiced extensively... in the context of progressively more challenging problems, projects, and standards for performance, personal and social responsibilities anchored through active involvement with diverse communities 
and real world challenges; and integrative learning demonstrated through the application of knowledge, skills, and responsibilities to new settings and complex problems. (Hovland, 2009, p. 5)

The studies in this section do not have consensus on a single definition of global learning (Elrod \& Hovland, 2011; Gibson, Rimmington, \& LandwherBrown, 2008; Hicks, 2003; Hovland, 2009; 2006; 2005a; 2005b) but give the responsibility to faculty for creating their own definitions and aligning the curricula and co-curricula to learning outcomes they create in their institutions. This is of importance to the dissertation because they show how important faculty members are and how their roles are firmly situated into the promotion of global learning in a university. The next passages expand on the importance of faculty.

\section{The Importance of Faculty}

Literature (Brewer \& Leask, 2013; Childress; 2008) shows faculty members are particularly important in the promotion of an internationalization plan at a university and in turn an internationalized curricula global learning is a part of. To begin with, an internationalized curricula brings about several challenges. Although on a conceptual level there can be a shared understanding of what is the internationalizing of the curricula amongst many peoples and institutions throughout the world, the context changes the meanings and activities of internationalization. In Europe, Internationalizing the curricula is often about understanding and cooperating with the rest of Europe for example. In the United States, the effects of a kind of parochialism, monoculturalism, and universalism can create obstacles to teaching and learning by informing flawed practices and concepts. In a university in the United States trying to internationalize, it is critical that faculty are 
there to address these challenges. This demands that they are part of the process and engaged (Brewer \& Leask, 2013).

Brewer and Leask (2013) also outline a framework for making the internationalization of the curricula successful that stresses the importance of faculty. It is suggested that: Institutions must have an internal understanding of what is internationalizing the curricula. Inventories of courses with international content can be used as a measuring device for internationalizing the curricula. Internationalizing the curricula demands that there be collaboration within and out of the university with other institutions, individuals, and resources. Faculty must be made responsible for it, compensated, and given ample space and time to reflect on what is an internationalized curricula. Learning outcomes of an internationalized curricula have to be clear and accessible. Students have to be a part of the process. Students need the metacognition to know what they are learning, what they need to learn, and the agency to address what they are not learning. Leaders need to balance a focus on international competitiveness as a goal with international cooperation. A variety of resources for internationalizing the curricula also have to be available to faculty and other leaders including funding (Brewer \& Leask, 2013).

Childress (2008) has explored how faculty engagement has affected the operationalization of university internationalizations plans. This study used a multicase design, interviews, focus groups, and document analysis. Childress developed a typology of effective engagement strategies after finding that Duke University and the University of Richmond used research, service and teaching at the campus, the region and internationally to promote faculty engagement in international activities. Childress also 
developed a model of faculty engagement based on the use of intentionality, investment, infrastructure, institutional networks, and individual support to promote internationalization.

The literature shows that faculty at a university have several rationales for promoting global learning in their curricula including the importance of global questions (Hovland, 2005b) and the globalization of content knowledge (Cornwell \& Stoddard, 1999). Global questions, of one sort or other, have always been the subject of academic study. Global questions are also useful frames that can bring coherence to the entire undergraduate learning experience. Global questions require students to connect, integrate, and act—whether they are biology, English, business, or international affairs majors, and whether they study abroad or stay on campus. The AACU used global problems to inform global learning and global questions as well for the heuristic and organizational value they held for the improvement of undergraduate learning (Hovland, 2005b).

Cornwell and Stoddard (1999) identified global trends and their impact on processes of academic knowledge production. These researchers argue that traditional knowledge production in an isolated United States is flawed and incomplete because the United States is part of larger world communities of multiple overlapping diasporas created by the evolving process of globalization where all knowledge is contested and subjective. All students need to be able to understand how knowledge production is globalized. These studies (Cornwell \& Stoddard, 1999; Hovland, 2005b) are important to this dissertation because these studies show that without a window into how faculty reflect on global learning knowledge and heuristics, global learning may be hindered by 
faculty who do not see knowledge as dynamic or the problems explored within their disciplines in dynamic frames that affect both questions and solutions. These studies are also promoting the idea that professors take an active dialogic approach in order for their students to learn which may not be the case.

The demands of promoting global learning necessitate that university faculty look at curricula in a new way and explore the promotion of certain competencies in order to take a critical approach (Marsella, 2007; Rauch \& Steiner, 2006). Marsella (2007) in a paper critical of traditional higher education and knowledge production claimed that the majority of challenges of global learning cannot be met within existing theories, assumptions, research methods or curricula. Marsella argued for mapping out three core elements of particular interest: the characteristics of the students, a philosophy of education, and the process and content of education. Marsella encourages the development of knowledge and skills that promote multidisciplinary, multilevel and multicultural approaches in research and education. Adequate training involves multidisciplinary methods, distance learning, new technologies, as well as requiring knowledge of global modeling, global communities and extensive field research. In practice, Marsella conclude, teaching and developing such strategies would require adjustments and changes at multiple levels in universities and colleges (Marsella, 2007). Rauch and Steiner (2006) argued that the type of critical approach used in global learning presupposes a set of specific competencies such as: systems thinking, team skills, the ability to deal with insecurity, integrated thinking, the readiness to cooperate, coping with change, creative and lateral thinking, and readiness to compromise. In Austria, for instance, global learning has been developed in classrooms via interactive exhibitions, 
workshops, classroom activities, and the use of auxiliary curricula materials for teachers. These works by Marsella (2007) and Rauch and Steiner (2006) are important to dissertation because it presents a major challenge to faculty and their universities that would have to be met with some kind of mechanism. Professional development which this dissertation explores and which is a common solution in many universities could be one such mechanism. The next few passages help explain the nature and importance of professional development.

\section{Importance of Professional Development}

Although representative of different aspects of the topic, a number of descriptors are used interchangeably with the term "faculty development." Among these phrases are: Instructional development, Professional development, Organizational development, Career development, and Personal development (Riegle, 1987). Several other terms in the literature are used to describe the professional development of university faculty. These include teacher development, professional faculty development, faculty professional development, educator development, adult learner development, and faculty development. Since this study focuses on university faculty as the professional, educator, adult learner, and teacher to be developed, the term professional development will be used synonymously with teacher development, professional faculty development, faculty professional development, educator development, adult learner development, and faculty development. Professional development in this sense will be defined according to definition of faculty development as any endeavor “. . . designed to improve faculty performance in all aspects of their professional lives - as scholars, advisers, academic leaders, and contributors to institutional decisions” (Nelson, 1983, p. 70). 
Professional development in general can occur in three ways. A faculty member could be allowed to be a self directed learner. A faculty member could be involved in formal professional development by attending a course or a workshop. In self directed learning and formal professional development the focus is on the individual faculty member improving themselves. Organizational development can involve both self directed learning and formal professional development (Caffarella \& Zinn, 1999). Organizational development has been defined as "a systematically planned change effort for the purpose of developing and implementing action strategies for organizational improvement" (Caffarella, 1994, p. 93). In this dissertation, faculty can be said to be involved in formal professional development through a workshop that is meant to improve faculty as individuals. The global learning workshops faculty members are attending in this dissertation can also be considered as organizational development. This is because these workshops are also part of the larger project of comprehensive internationalization that seeks to transform the entire university as an organization. These specific workshops are discussed in detail in the next chapter.

Sikes and Barrett (1976) argued that the goal of professional development for faculty is to make “. . college teaching more successful and more satisfying” (p. 1). In the past, professional development did not seem necessary because there was the common belief that if one attended school, they could teach what they learned to others. This view assumed a lack of metacognition when it came to teaching and learning in that it presupposed that the student learned their content fully and were taught the way to teach it by simply mimicking their professor (Mclean, Cilliers, \& Van Wyk, 2008). Eventually, the increasingly speed of change when it came to knowledge production 
along with other factors meant that a professor could not rely on knowledge they learned in the past and promoted the need for professors to catch up on learning on a regular basis. Institutions realized that professional development kept an institution and the faculty from being obsolete. In America, professional development could take the form of sabbatical leaves from the university where faculty could take time to learn on their own or by attending another institution (Riegle 1987; Schuster 1990). Professional development of faculty was for some time narrowly viewed as typically being concerned with the advancement of subject matter competence and the mastery of one's own discipline as it related to teaching (Sullivan 1983). Eventually more formal structures of professional development grew within universities to encompass more than just the promotion of content knowledge or the teaching of it to include other matters such as administration. Professional development was offered within the universities. Funding of professional development also grew (Camblin \& Steger, 2000).

Navarro (2004) researched the factors that affect participation of faculty in the internationalization of the curricula. The research found eight core factors were important: (a) context, culture, and environment; (b) support by administration; (c) priorities of faculty (including the value they assign to internationalization); (d) state of the curricula and available tools/strategies for curricula change; (e) incentives given for faculty participation; (f) pedagogy, technical, international, and other professional development opportunities available to faculty; (g) resources available; and (h) perceived needs. A key strategy for enhancing faculty participation in internationalization is the promotion of several professional development opportunities for faculty. This study supports this dissertation in highlighting the importance of professional development of 
faculty towards the internationalizing of the curricula. The dissertation addresses a gap in understanding faculty reaction to global learning specifically.

In order to promote internationalization, some institutions have sent faculty to teach in another country as a form of professional development. Research for example has explored faculty beliefs about Costa Rica before participating in international professional development (Harder, Lamm, Roberts, Navarro, \& Ricketts, 2012). The problem with this research is that it does not focus on global learning specifically or transformational learning. In essence, international professional development is a faculty version of study abroad. This dissertation is focused on professional development within a faculty members' home institution.

The impact of professional development can be mitigated by several factors. Zinn (1997) focused on key factors supporting and impeding teacher leadership. One of her major conclusions was that there are four domains within which these supports and barriers are clustered: The first are people and interpersonal relationships, the second are institutional structures, the third are personal considerations and commitments, and the fourth are intellectual and psychosocial characteristics. These factors could be important in the context of professional development in FIU because they allow for critical reflection by the faculty. Through professional development the university has an institutional structure that can support faculty across the university move beyond the barriers of professional and personal considerations and commitments to make a space friendly for people to develop interpersonal relationships with other faculty who have the intellectual and psychosocial characteristics to share complex forms of reflection about global learning. 
Faculty reflecting on practice is a concept that originated in the enlightenment (Cranton, 2001). University faculty can have a conception or belief about teaching (Kember, 1997). A perspective on teaching is an interrelated set of beliefs, intentions, and actions linked to knowledge, learning, and the role of a teacher (Pratt, 1992). Two conceptions were found in the literature review of thirteen articles by Kember (1997). The first conception is a teacher perspective centered on how the content knowledge can be transmitted and is centered on the teacher because learning depends on the skills and strategies of the teacher in promoting the content knowledge. The student is not taken into account. Another teacher perspective is student and learning centered. It is based on an interaction between the student and the teacher where the teacher must help build on the students prior knowledge. It is student centered because the student must construct their own knowledge (Kember, 1997). A teaching perspective can be the lens through which a faculty member sees everything (Pratt, 2002). The practice of reflecting on perspective demands that faculty stand outside themselves to explore the assumptions that support what they teach and how they teach it (Brookfield, 1995). Having faculty reflect on their teaching is important because their perspective affects what is taught, how it is taught, and various other practices and activities in higher education (Cranston, 2001) and the work of the rest of the university community (Kember, Kwan, \& Ledesma, 2001).

A teaching perspective however can be difficult for a faculty member to discern but it is the lens through which a faculty member sees a phenomenon and not the actual seeing of a phenomenon (Pratt, 2002). The lens can be effected by faculty members own autobiographies, their experiences as adult learners, the lens of students' eyes, the way 
they see their colleagues' perceptions, and the way they view educational literature (Brookfield, 2002). Research in Europe, Asia, and other countries (Eley, 2006) has focused on ascertaining teaching perspectives and categorizing them (Pratt, 2002).

Pratt (2002) developed an instrument called the teaching perspective inventory that is used to categorize a teaching perspective into a transmission, apprenticeship, developmental, nurturing, or social reform perspective. In a transmission perspective the faculty are masters of authorized or legitimated knowledge and use preparation to develop a way to guide students through formal activities and tasks in a systematic way that leads to their mastery of content knowledge. In the developmental perspective, the faculty member uses a constructivist perspective to build bridges from a students' current way of thinking to more increasingly complex and sophisticated ways of thinking. The internship perspective has faculty helping students learn by involving them in the performance of real life tasks, skills, and situated in authentic communities of practice and by making the meaning behind the performance understandable. The nurturing perspective assumes that long term academic achievement and persistence is promoted by the heart as much as the head. The social reform perspective is oriented towards bringing about social change. Many educators find their own perspective as a combination of several of these classifications.

Many universities are using professional development as vehicles to promote reflection and dialogue about these teaching perspectives (Hubbal et al., 2006). The literature on reflection (Brookfield, 1995; Cranston, 2001; Kember, 1997) however does not deal with global learning specifically. Research on reflection is also often limited to education in a general sense (Hoffman-Kipp, Artiles, \& López-Torres, 2003; Pratt, 2002). 
The work that categorizes teacher perspectives (Pratt, 2002) deals with reflection in simpler and less personal sense than it would be dealt with in this dissertation. Reflection in this dissertation is seen in a more complex sense that the faculty will need to express because the process of internationalization and the promotion of global learning already leans faculty towards complex sets of perspectives. FIU's use of global learning for example is meant to lead to global citizenship which can promote social reform when students are engaged in solving global problems, the use of authentic contexts as students act locally and think globally, and is developmental as faculty and students have to interact to create knowledge by building on prior knowledge.

The work of Schon (1983) and Thornton (1988) are important to this dissertation because these studies help outline the importance of faculty member towards the promotion of curricula. Critical reflection by faculty members on and during their practice helps faculty resolve issues in the curricula. Faculty members can reflect on their own practice as they perform it and others can learn from the performance. As they reflect on their action they are resolving problems and issues within the curricula that they may only be able to verbalize after the moment (Schon, 1983). Reflection also helps faculty members create a consonant relationship between what they intend to teach, what they actually teach, and what the students actually learn. When these relationships are not consonant, there is a confusing mix of three curriculums at work in a classroom. This is the official curriculum, the curriculum that the faculty member strived to teach, and what is taught and learned (Thornton, 1988). These studies (Schon, 1983; Thornton, 1988) are important to the dissertation because they allow for the possibility that faculty can reflect in and on their practice both as individuals and collectively during professional 
development and the work of the dissertation will help them verbalize and learn from these reflections. Professional development is also a space where faculty members could practice, reconcile and make consonant the relationship between what they intend to teach, what they actually, and what is actually learned with the help of other faculty members. Faculty members in this space are an influential circle of learners and teacher who can affect those within and outside the circle particularly in terms of the curriculum consonance of global learning.

Dewey (1933) was an influential voice in promoting the need for teachers to reflect on their teaching and learning. Hoffman-Kipp, Artiles, and López-Torres (2003) argued that teacher reflection must go beyond simply reflecting on practice and learning to praxis. Reflecting on teaching in a technical way as an isolated skill is promoted by the demands of testing, standardization, and other forces in our schools. Reflecting on teaching and learning is not enough because it may simply help an educator teach a flawed curriculum that disempowers students as well as the educators themselves. Reflecting on teaching and learning individually as a phenomenon allows for a more holistic personal understanding of the nature of teaching and learning but that knowledge still remains for the most part isolated in the individual teacher. Critical reflection is important because it highlights the structures that mark the way knowledge is produced, taught, and learned. Critical reflection is limited when it is left in the mind of the individual teacher. Situating critical reflection within a community of educators where educators share with each other their reflections and distribute them through communication, symbols, and the use of activities and artifacts allows for a growing social awareness of problems. Reflection as a metacognitive, social practice can allow 
for educators to not only become social aware but to engage in the improvement of their worlds.

The work of Hoffman-Kipp et al. (2003) is critical to the work of this dissertation in that it suggests that reflection in professional development can lead to praxis. Arguably, the conditions for reflection as praxis exist in the work of the global learning professional development workshops at FIU. The impact faculty members have on internationalization and global learning can be seen in part as the praxis of that reflection that a larger community of scholars can be made aware of through this dissertation. Reflection is critical to transformational learning (Mezirow, 1978, 1991, 1996, 2000). King (2002) in a mixed method study using surveys and interviews has studied the way learning activities are a factor in the reflection promoted in the professional development of faculty. King (2002) argued that if the right needs are met for faculty, a professional development workshop can lead to transformational learning. This study was not in the context of internationalization and the promotion of global learning in professional development. This dissertation seeks to address that gap in the literature. King (2002) showed that a survey along with an interview guide could be developed by a researcher and used as both a data collection tool and a way to filter a population to find out who experienced transformational learning. This dissertation will use an original survey (See Appendix A) and original interview guide (See Appendix B) developed by the researcher in consultation with his dissertation committee in a quantitative and then qualitative stage.

Cranston (1996) explores the many facets of professional development of adult learners and the perspectives that drive professional development, to explain how adult 
learners become transformational learners. The research is based on a conceptual analysis of the professional development literature and by exploring the narratives of several adult learners. An argument is made that the different orientations professional development is based on needs to be rethought. The technical perspective looks at professional development as the acquisition of certain skills, capacities, and forms of knowledge. It is flawed because it does not challenge the status quo. The humanist perspective to professional development looks at teaching as a primarily nurturing and developmental act focused on the needs of the student. The educator becomes subservient to the learning of the student and once educated, it is up to the student to have agency and use the knowledge in the way they see fit. The social activist perspective looks at professional development as a political act. A certain kind of sophistication is needed to understand, accept, question, use, and balance the multiple roles of educator, learner, and activist within the social activist adult learners' identity effectively. The postmodern perspective looks at professional development as a set of norms to be challenged. The postmodern perspective leaves no clear context for critical reflection because postmodernism promote a fragmentation in meaning and a lack of cohesion.

Cranston's (1996) work is important to this dissertation because it helps clarify the orientation of professional development. In the dissertation, professional development for global learning may touch on aspects of the several professional development orientations mentioned, but the faculty member who is promoting global learning for global citizenship is accepting a social activist role and being asked to teach a kind of social activism to their students. 
Cranston (1996) also provides a model that explains how transformational learning can lead to social change. A model is outlined where the individual learners have to confront the influences of his or her own individual characteristics (such as personality traits), as well as the influences of the contexts of their teaching, organization, and culture. The interplay between self-directed learning, critical reflection, and transformational learning within that adult learner, allows that person to make change on the individual, organizational, and social levels (Cranston, 1996). This research is important to the dissertation because it supports what internationalization leaders and global learning promoters at FIU seek to do with professional development. Professional development in FIU is arguably meant to create change at the individual, organizational, and social level. This dissertation measures a part of that changing by focusing on transformational learning.

Cranston (1996) argued for the importance of critical reflection in professional development because it is a primary element in transformational learning. Strategies for promoting critical reflection in professional development are outlined such as the promotion of discussion, the articulating of assumptions, determining sources and consequences of assumptions, the questioning of assumptions, and the imagining of alternatives. This study is important to the dissertation because it outlines strategies for promoting critical reflection for transformational learning that are inherent in the work of global learning professional development for faculty at FIU and which help guide the modification of the instruments used in this dissertation to ascertain the transformational learning in global learning professional development this dissertation uses. 


\section{Transformational Learning}

Mezirow (1978) conducted a qualitative study to explore the needs of women in the United States who were resuming employment or education after an extended period time of out of the university or out of the workforce in order to understand what are the factors that help or facilitate their progress in completing school or reintegrating themselves back into work life. The study explored 12 reentry college programs with 83 women in four states. Further follow up studies were done through telephone and mail inquiry of several reentry programs nationwide. The research showed that respondents had undergone a personal transformation after becoming critically aware of what they expected of themselves and what was expected of themselves by society. These women decided to make changes in their assumptions and in their beliefs. Mezirow identified 10 phases that these respondents could have experienced in this process of transformational learning (Mezirow, 1978). Mezirow’s (1978; 1990, 1994) work on the 10 phases of transformational learning is organized around larger ideas that include disorienting dilemmas, critical reflection, rational dialogue (discourse) and action which Herbers (1998) in a study exploring the transformational learning of preservice teachers summarized as key components of a perspective transformation. The view of transformational learning phases as components of perspective transformation has informed the work of several researchers including Glisczinski (2007) and Caruana (2011).

Transformational learning theory specifically is when 'learning is understood as the process of using a prior interpretation to construe a new or revised interpretation of the meaning of one's experience in order to guide future action' (Mezirow, 1996, p. 162). 
Since Mezirow’s (1978) initial work, Mezirow has continued to refine his approach to transformational learning and its language by emphasizing the importance of different forms of critical reflection, arguing that transformation can occur at different paces, and arguing for the important of relationships and dialogue (Mezirow, 2000). Adult learners have meaning perspectives. A meaning perspective is “a habitual set of expectations that constitutes an orienting frame of reference that we use in projecting our symbolic models and that serves as a (usually tacit) belief system for interpreting and evaluating the meaning of experience” (Mezirow, 1991, p. 42). A perspective that is concretely manifested through practice into "specific sets of knowledge, beliefs, value judgments, feeling, and assumptions" is said to be a meaning scheme” (p. 44). Meaning schemes can be evidence of a meaning perspective. An adult learner can have several kinds of meaning perspectives to use to view a problem. A person can have an epistemic meaning perspective based on how they understand the nature of knowledge. A sociolinguistic perspective uses a person's understanding of social norms, cultural codes, and the way they use language to view problems. Psychological meaning perspectives use a person's understanding of themselves as individuals to view a problem. Our meaning perspectives are considered distorted when they are underdeveloped. This underdevelopment leads to learners being limited in terms of what they see of reality, keeps them from differentiating aspects of reality, causes them to be closed to other ways of seeing reality, or causes them to be unable to fully integrate reality into their experiences (Mezirow, 1991).

When adult learners confront something in reality that does not easily conform to their meaning perspectives or meaning schemes, it can become a problem or disorienting 
dilemma that causes them to go into critical reflection. A learner can critically reflect on the content of a problem, reflect on the process used to solve the problem, and/or the learner can reflect on the validity of how the problem is framed. Critical reflection does not always lead to transformational learning. Critical reflection may in fact simply validate or support a perspective a learner has. Critical reflection leads to transformational learning when it leads to a transformation in perspective. This change in perspective can happen abruptly or gradually over time (Mezirow, 1991).

In Mezirow’s (1978; 1991; 1996) theory of transformational learning, for a transformation to occur, a person must go through a variation several phases within the larger themes of experience, critical reflection, and rational discourse that make up the theory of transformational learning. In terms of making meaning from experience, a person must encounter a disorienting dilemma. This is followed by critical reflection on the experience that can lead a self-examination with feelings of guilt or shame and/or a critical assessment of assumptions. Critical reflection can only lead to transformational learning if it is followed by one of the phases of rational discourse. In rational discourse, an individual can have a recognition that one's discontent and the process of transformation are shared and that others have negotiated a similar change. An individual begins an exploration of options for new roles, relationships and actions.

There is also a need for action in transformational learning that can include a planning of a course of action; acquisition of knowledge and skills for implementing one's plans; a building of competence and self-confidence in new roles and relationships; and finally a reintegration into one's life on the basis of conditions dictated by one's new 
perspective (Mezirow, 1978, 1991, 1996). Mezirow’s (1978) conception of

transformational learning focuses on the adult learner and became very popular in the last few decades (Taylor, 2007, 2008). Mezirow’s (1978) approach to transformational learning however is not a singular conception of transformative education; it is built upon the works of several other researchers (Kitchenham, 2008). The influences on Mezirow's (1978) early theory of transformative learning included Dewey (1910), Kuhn (1962), Friere (1970), and Habermas (1971). Dewey (1910) promoted the need for reflection in Education. Dewey argued that any belief or thought had to be consistently reflected up in order to understand and evaluate the grounds that support it and to appreciates its consequences.

Kuhn (1962) explored how major achievements or ideas in the history of science took hold. These achievements, ideas, and concepts that became universally accepted by practitioners Kuhn referred to as paradigms and the process of adopting these paradigms became paradigmatic transformation. These paradigms included the nature of electricity and the notion of the Earth revolving around the sun. These paradigms were not the result of a progression of ideas but a revolution in thought because they showed the limits of previous paradigms while connecting several ideas together enough so as to attract the interest of practitioners away from other subjects to explore the problems and solutions the paradigms modeled (Kuhn, 1962). A perspective transformation is akin to a paradigm transformation because it shows the limits of one's prior beliefs while setting the stage for changing one’s thought and future action (Kitchenham, 2008).

Freire's (1970) critiqued a form of Education where the student was imputed knowledge that a teacher deemed them worthy of receiving. This banking education gave 
power to the teacher and did not allow the student to gain or develop knowledge on their own that would be recognized or legitimated and would allow the student to change their circumstances. Freire suggested that teachers instead hold equal footing with their students in order to work with them to gain a critical consciousness called concientization where together the teacher and student could question the oppressive aspects of the student's reality caused by the contradictory forces within the social, political, and economic realms of their world. This education would be liberating because the teacher would help the student take agency in gaining their own knowledge and changing their world (Friere, 1970). Concientization or a raising of a critical consciousness in a student is critical to transformational learning because it is through reflection that one can see the limits of one's beliefs and the need to change (Kitchenham, 2008).

Habermas’s (1971) argued for three domains of learning. In the technical domain of learning, instrumental learning is and teaching done through rote and is governed by specific rules. In the practical domain, learning and teaching is informed by social norms. In the emancipatory domain of learning, one considers their own beliefs about what they are learning or teaching (Habermas, 1971). Emancipatory learning and transformational learning share the need to question beliefs and the need to communicate (Kitchenham, 2008). The domains of learning (Habermas (1971) were later used by Mezirow (1985) to develop his own models of learning. These models called the instrumental, dialogic, and self-reflective types of learning influence perspective transformation. With the instrumental type of learning, the learner must ask what is the best way to learn new information. The dialogic learner asks where and when is it best to learn this new information. The self-reflective learning asks why they are learning this new information. 
With each type of learning the learner could try to learn within meaning schemes, learn new meaning schemes, or learn through meaning transformation (Kitchenham, 2008). Mezirow’s (1978) transformational learning theory competes with other conceptions of transformative learning including the psycho-developmental approach, the psychoanalytical approach, the social emancipatory approach, the race-centric approach, the cultural-spiritual approach, neurobiological approach, and the planetary approach (Taylor, 2007; 2008). A psycho-developmental approach is where one transforms over the span of a lifetime often through relationships, interactions with a context, and through a holistic understanding of the making and knowing of knowledge. Level of rationality and communication is not the only variables needed for transformation. Transformation can occur when one is first developmentally ready to be able to transform (Daloz, 1986; Kegan, 1994).

In psychoanalytical approaches, symbols, archetypes, and in depth exploration of one’s personality and psychology is used (Boyd \& Meyers, 1988). Transformation happens through an exploration of psychological characteristics that make up an individual's identity and the development or discovery of a greater sense of confidence, responsibility, and creativity (Boyd \& Meyers, 1988; Cranton, 2000; Dirkx, 2000). The social emancipatory approach is an expansion of the work of Friere (1971). The focus is more on social change and less on individual change. A person develops a mindset where other people are subjects and not objects, there is a sense that people are constantly acting and responding to change, and the goal is to recognize the power and knowledge of others instead of transferring knowledge to them. Teaching is done to demythicize realty, to support the agency of others, and to promote critical reflection. This teaching 
emancipates groups of people from the limiting prior knowledge these learners faced before (Friere, 1971, 1984; Friere \& Macedo, 1995).

Race-centric approaches explore the need for transformation through the prism of race and other non-Eurocentric approaches. Much of this work focuses on the transformation of people of African descent as an essential part of African culture. The focus is not on the individual but on empowerment through membership in part of the larger community, a connection to the universe, and through the recognizing of once silenced voices and learning to navigate and thrive through various cultures (Williams, 2003; Sheare, 1994; Johnson-Bailey \& Alfred, 2006; Brookfield; 2003). A culturalspiritual view of transformative learning explores the way people use storytelling, spirituality, and group inquiry in order to help themselves and others deal with their experiences and imagine alternatives. People explore how to use narrative (transformative knowledge) to make connections between themselves and social structures. How much what they learn is based on how their identities intersect in different positionalities (Brooks, 2000; Tisdell, 2003; 2005).

The neurobiological perspective of transformative learning uses the current science of the brain to argue that transformation is informed by changes in the physical structures of the brain, that learning often comes with some discomfort, and that transformational learning actually changes the brain. These changes are strengthened by emotions, the use of the senses, kinesthetic experiences, an appreciation of the difference between male and female neurobiology, and education that uses discourses and knowledge bases of neurobiological systems (Janik, 2005). A planetary view of transformative learning seeks to change the way human beings see their world by 
changing their relationship to themselves, to other humans, to nature, to the ecology, and to the various systems of the world. By promoting various forms of interconnectedness, the individual is encourage to develop a world view that integrates various dimensions of life and which creates a narrative that challenges various dysfunctional values (O'Sullivan, 1999).

In the first section of this chapter, the major concepts of this dissertation were explored. First global learning was situated within the context of internationalization and comprehensive internationalization. Then the importance of faculty was explored. This was followed by the importance of professional developments. The concept of transformational learning was then explored. Since this dissertation is focused on faculty and global learning and transformation, the next section of this chapter consists of a literature review of research on faculty and global learning and a literature review on research on transformation learning and global learning.

\section{Literature on Faculty and Global Learning}

For university faculty, the promotion of global learning demands a change in the way a discipline is taught in the curricula. Globalization and global learning has had a huge affect on the disciplines students encounter. Religious studies programs often dealt with the dichotomy of east and west religious cultures for example. Beloit College moved from an east to west dichotomy to a local and global orientation in their religious study program. Global learning students bring what they have learned before to an experiential project and move from learning about others to learning from others (Duerst-Lahti, 2005). Noting how globalization had changed the perception of the nation state concept forced stakeholders in the American Studies discipline to rethink what was American. In 
a program at Oklahoma State University, the web was used to explore how local spaces were changed. American Studies students were shown how much what was local was also American, global and a part of their everyday lives (Takacs, 2008). These studies highlight how faculty must incorporate global learning into the content and focuses of their disciplines and are anecdotal in nature (Duerst-Lahti, 2005; Takacs, 2008). These studies are important to this dissertation however because they highlight that there is a need to promote global learning to faculty and that global learning can potentially change the way a faculty member looks at their disciplinary content. This reaction and reflection in part is something this dissertation seeks to explore with a more rigorous methodology.

Several reports discuss the implementation of successful teaching strategies for global learning into existing courses (Barbiero, 2008; Lenz \& Warner, 2011; Perry \& Mander, 2005; White, 2005). University faculty are successful with global learning when they are able to show how a local problem is really a global problem and design a curricula around this frame that incorporates other members of the university and world community. Some of the more successful comprehensive internationalization/ global learning projects have been in subjects that are naturally global for example. The fight against disease and the promotion of health knows no borders. Barbiero (2008) presents a review of an undergraduate global health curricula implemented at the George Washington University School of Public Health and Health Services. It is in concert with the framework and principles of the Association of American Colleges and Universities and their vision of Shared Futures of Global Learning and Social Responsibility. The rationale for a deep and broad undergraduate public health curricula, which includes a global health component, is made clear with how many health issues spread with 
globalization (Barbieri, 2008). Nursing students from San Francisco State University claimed to have gained an international frame of reference working in study abroad programs in places like Thailand and the United Kingdom (Perry \& Mander, 2005).University faculty members are also challenged to develop a global learning curricula that is inclusive to the entire student body by using strategies that mitigate gatekeeping barriers. Students who cannot afford study abroad cultural immersion courses for example can still be global learners through the use of carefully planned interactions at the local level or through the use of technology. White (2005) offers a description of a web based class for global health for students that cannot afford international travel for example (White, 2005). Community health assessment with a local health department gives another alternative to study abroad for undergraduate nursing student in some programs (Lenz \& Warner, 2011).These studies that discuss the implementation of teaching strategies for global learning into existing courses (Barbiero, 2008; Lenz \& Warner, 2011; Perry \& Mander, 2005; White, 2005) do not focus on how faculty were transformed by global learning or the role of faculty development in that process. Another gap in the literature is that there is no link as to how that transformation within faculty applied to their teaching of global curricula. This dissertation would fill that gap with qualitative data through personal reflections given by faculty in their own voices.

\section{Literature on Transformational Learning and Global Learning}

Exploring the literature on transformational learning related in some way to global learning reveals that much of it is not focused on whether university faculty are transformed as learners by their exposure to global learning curricula in professional 
development. Most of the literature focuses on students and other adult learners (Cohen, 2008; Cummins \& Sayers 1997; Dahl, 2009; Dodson, 2009; Freeman \& Knight, 2007; Geelhoed, 2009; Henderson, 2002; Kean, 2010; Rehm, 2009; Schnick \& Petrequin, 2007; Silveira, 2007; Taylor, 1994). A global perspective was developed amongst business students in various backgrounds and areas through email exchanges while learning about business techniques and the cultures of Canada and Kazakhstan (Freeman \& Knight, 2007). Cohen (2008) argued that making secondary school available to everyone would help developing countries and the world. Global learning is as dependent on how much learning people have throughout the world (Cohen, 2008).

An interpretative case study showed that undergraduate and recent graduates who had served in a student organization focused on global social justice had experienced sustained transformative learning as a result of their activism (Kean, 2010). Transformational learning and international healthcare education were used as concepts in a study that explored the transformational learning and experiences of students in multiple international healthcare missions in the developing world (Geelhoed, 2009). Dahl (2009) explored the importance of camps as a particular type of thinking space. Camps act as a youth centered grey zone between many institutions. Global citizenship and peace related learning is promoted through language and cultural immersion in Concordia Language Villages. This study looks at changes in attitudes, perceptions, and the value of certain learning opportunities (Dahl, 2009).

Rehm conducted a study of an online community of learning created to help the members of a large international organization collaborate and exchange knowledge in order to train themselves for work in a global organization (2009). Transformational 
learning theory was used to explore how fourteen participants negotiated disorienting dilemmas in their lives in a study based on the notion that in our modern global world people would face many challenges (Dodson, 2009). Researchers also argue for using transformational learning theory to support or describe other concepts. Transformational learning can complement transformational change theory to help people seeking transformational change in organizations (Henderson, 2002). Transformational learning could also be used to explain the process of intercultural competence gained by Americans living abroad (Taylor, 1994).

Some Educators have suggested that global learning can lead to transformational learning. Educators claim that students have been transformed into global citizens by using art education for example and the pedagogy of Freire to promote social justice through community service learning (Silveira, 2007). Questioning industrial capitalism and the concept of the American dream has also been the starting point for courses that try to promote global learning outcomes. These educators however do not describe any particular methodology for determining that their students were transformed and seem to be giving anecdotal evidence (Schnick \& Petrequin, 2007; Silveira, 2007). The literature that deals with a transformational learning pedagogy that can be related to some aspect of global learning in some ways falls in the categories of suggestions (Cummins \& Sayers, 1997; Meyers, 2006; Meyers 2008), critiques (Bowers, 2005; Clover, 1995), evaluations (Fetherston \& Kelly, 2007; Hanson, 2010; Kourtis, Kazoullis, \& Skourtou, 2004), and models (Brown, 2004; Nagda, Gurin, \& Lopez, 2003).

Some of the literature that deals with transformative pedagogy for example suggests ways in which global learning networks can be used and conditions for it. 
Cummins and Sayers (1997) for example argued that transformative pedagogy requires students to become active learners in the classroom. Transformative pedagogy also demands that professors frequently use strategies such as collaborative learning, problem based instruction, discussions, or role plays to promote engagement (Cummins \& Sayers 1997). Meyers (2006) developed the following series of steps for faculty members to implement transformative pedagogy. These steps include creating a safe environment in class and encouraging students to think about their experiences, beliefs, and biases. Using teaching strategies that promote student engagement and participation, posing real-world problems that address societal inequalities, and helping students implement actionoriented solutions are also important steps (Meyers, 2006). Meyers (2008) also suggests that teaching online can help transformative pedagogy practitioners maintain their commitment to social justice. Many activities that generate social action are inherently portable and can occur outside of regular class meeting times. Examples of these activities include writing advocacy editorials to local or university newspapers, creating or participating in relevant co-curricular or communities activities, or organizing demonstrations on campus or in students' communities (Meyers 2008). These suggestions do not offer the reflections of university faculty (Cummins \& Sayers, 1997; Meyers, 2006; Meyers 2008). This is a gap that the dissertation addresses through the answers gathered in the open questions in the instrument used for data collection.

Critique of transformational learning pedagogy often deals with how it has been used or interpreted (Bowers, 2005; Clover, 1995). Clover (1995) gave a gendered critique of the theory and practice of popular education and of environmental policy and planning processes. The kind of transformational learning in this education gives visibility to the 
means by which women's world views and ways of knowing and doing motivate their environmental and educational work. With reference to experiences women had in India, Bolivia, El Salvador, Brazil, and Canada, a case is made for the recognition of nonviolent, but holistic resistance strategies. These are based on women’s approaches to environmental action learning, to change attitudes, behavior and responsibility for personal and social transformation and action to inhibit the continued degradation of human support systems (Clover, 1995).

In another study, Bowers (2005) argued that Friere and other proponents of a transformative learning pedagogy are in fact colonizing indigenous people with neoliberalism and conservativism. These proponents arguably attack the commons, promote individualism, and devalue traditional knowledge by having students rename knowledge to counteract banking pedagogy. Bowers argued that Friere was beginning to understand this issue as it plays out in ecology discussions before his death. The commons is resistance to market capitalism and progressivism. Transformational pedagogy can for example make students move away from traditional knowledge that promotes public access to water and subsistence culture. When water resources are privatized and the water is bottled to sell as a commodity elsewhere, students may rename and create new knowledge that is not as effective for the ecology as the traditional knowledge (Bowers, 2005). These are conceptual and theoretical studies (Bowers, 2005; Clover, 1995) may indirectly address global learning issues but focuses more on activism beyond the classroom. These studies are not based on global learning curricula that has been reflected upon in professional development. This dissertation explores global learning and transformational learning in practice. 
Several studies evaluate a transformational learning pedagogy used in a course that can be related in some way to global learning. (Fetherston \& Kelly, 2007; Hanson, 2010; Kourtis et al., 2004). An evaluation study of a two part global health course in a university in Canada for example explored transformative learning theory. The focus was on the work of Friere and Mezirow in regards to social transformation and pedagogy. Canadian style internationalization at the undergraduate level marked this course which was a mix of study abroad and traditional campus bound work. Active learning was used in the course. In Nicaragua where students traveled to, immersion and reflection helped change students. Reading was a major focus in the first part of the course however and students often felt conflicts with the university (Hanson, 2010).

Transformative learning theory was used with cooperative learning in a conflict resolution course aimed at peace building. The classes were taught in a traditional manner in the first hour and in the new pedagogy in the last two hours. Portfolios, diaries, and essays were used. Promoting praxis and critical reflection was important to the researchers but the habitus of students affected how students viewed the class. Some students were dissatisfied with the new pedagogy and did not understand the point of the course. Some students changed their views but did not necessarily transform. Toxic relationships in student groups that had to cooperate to do jigsaw presentations, projects, and activities also affected learning and satisfaction with the course negatively. The promotion of literature in the traditional part of the class was important for students and their understanding. Grounded theory, pre and post survey, and interviews were used to do this study (Fetherston \& Kelly, 2007). 
Kourtis et al. (2004) explore how the internet can open up spaces for Progressive, Constructivist, and Transformative Pedagogy. An internet based sister class project designed for the teaching of English as foreign language and Greek as a second language is used as an example. The use of the Internet in a learning environment means that it is usually "added" to something that has existed before. In the classroom, the internet is added to community of the classroom with a teacher and the students. The way the lesson is carried out depends on a variety of variables. The pedagogy that is used can be classified into one of three general orientations: traditional, constructivist or transformative or can be a combination of all three. If this classroom is a foreign language classroom, teaching and learning can follow a particular language learning methodology or approach or most usually is a synthesis of methodologies or approaches. When the Internet is "added" to the environment of the classroom, changes inevitably take place. The degree of these changes depends on how the Internet since the internet can be used in very traditional or in innovative new ways (Kourtis et al., 2004).

These evaluation studies (Fetherston \& Kelly, 2007; Hanson, 2010; Kourtis et al., 2004) are related in some way to global learning but are not a part of a deliberate global learning and comprehensive internationalization plan. The main focus of these studies is not on global learning but primarily on transformational learning. In the literature, many researchers develop models of learning that seem to incorporate aspects of transformative pedagogy and aspects of global learning. These models are often used to describe issues or to interpret what goes on in a course. (Brown, 2004; Nagda et al., 2003).

Nagda, et al. (2003) suggest a theoretical model of engaged learning for democracy and justice. This model draws from multicultural education, critical 
pedagogy, Freireian dialogic education, and Kolb’s active, experiential learning. Engaged learning is defined by the researchers as applying concepts and ideas from the classroom to out-of-class cognition and action. This empirical investigation examined the impact of a course focusing on intergroup relations and social conflict. Participation in this course is shown to increase students' structural attributions for racial/ethnic inequality and socio-historical causation. Course participation also increases students' action orientation away from individual blaming to individual action toward institutional targets, and institutional and societal change. On pre- and post-test measures, engaged learning is demonstrated to mediate the impact of course content and active pedagogy on students' active thinking and understanding of socio-historical causation as well as students' action strategies that promote more tolerance. On post-test only measures, engaged learning mediated effects on socio-structural understanding, understanding others, and learning about conflict. The importance of content, active pedagogy, and engaged learning, and implications for future research and practice on teaching about democracy and social justice are explored (Nagda et al., 2003). In another study, the three theoretical perspectives of Adult Learning Theory, Transformative Learning Theory, and Critical Social Theory were interwoven with the three pedagogical strategies of critical reflection, rational discourse, and policy praxis in a model used to increase awareness, acknowledgment, and action within the preparation programs for Educational leaders (Brown, 2004). These studies (Brown, 2004; Nagda et al. 2003) however do not directly address global learning directly, the reflections of faculty, the role of professional development, and are mostly conceptual and theoretical. 


\section{Summary}

This literature review was organized into two main sections. The first section centered around the concepts of situating global learning into context, the importance of faculty, the importance of professional developments, and transformational learning. This section helped stressed the importance of global learning, university faculty, and the power of reflection in professional development as part of the internationalization process. The second section of this chapter explored the literature on faculty and global learning and the literature on transformational learning and global learning. Gaps in the literature revealed that despite the potential for global learning related professional development to lead to transformational learning and the development of methods that could measure that potential, there is no quantitative or qualitative research found by the researcher that examines if or how university faculty are transformed by global learning in professional development or how it could impact their teaching.

\section{Overview of Succeeding Chapter}

The next chapter of this proposal explains the methods of a study that explored if and how university faculty can see being taught global learning curricula as transformational learning after a faculty development workshop. 


\section{CHAPTER III}

\section{METHOD}

This chapter is organized into several sections. First there is a restatement of the purpose and research questions of the study. This is followed by the description of the research design that was used, the rationale for the research design, a description of Florida International University’s (FIU) faculty, FIU’s global learning professional development workshops, and a description of the population. This is followed by explanations of the sampling, data collection, and data analysis done first in the quantitative stage, and then in the qualitative stage of this study. Integrity measures and data management procedures are then explained. This chapter ends with a summary and an overview of the succeeding chapter.

\section{Purpose}

The purpose of this study was to expand on the literature on global learning and professional development by exploring whether university faculty members who have attended global learning professional development workshops perceive global learning as transformational learning, and if they do, how do these faculty members see global learning as transformational, and how this transformational learning informs their teaching.

\section{Research Questions}

This study sought to answer these main research questions: Do university faculty members who have attended global learning professional development workshops report a transformational learning experience? How do university faculty members who have attended global learning professional development workshops perceive global learning 
curricula as transformational learning? How do university faculty members who have attended a global learning professional development workshop and report having had a transformational learning experience apply this transformation to their teaching?

\section{Research Design}

This study used a single case study design. A case study is an in depth exploration of an activity, event, process or individuals that make up a bounded system (Creswell, 1998). This study used FIU's global learning professional development workshop as an exemplary case and the primary unit of analysis. The sources of the data for this case study came from surveys (See Appendix A) and interviews (See Appendix B). According to Yin (2004), case studies are suited to answering how and why questions for real life phenomenon. This design was chosen because in depth qualitative analysis of a single case can add insight into the issue of how global learning as transformational learning is perceived by faculty members and how global learning as transformational learning can inform instruction.

\section{FIU Faculty}

As of November 14, 2014, there was a total of 1,208 faculty members at FIU made up of 255 full professors, 299 associate professors,346 assistant professors, 281 faculty members ranked as instructor/lecturer, and 27 faculty members listed without a rank There were 515 female faculty members and 693 male faculty members. Forty percent of all faculty members had tenured status A terminal degree was held by $99 \%$ of all faculty members who were tenured or who were on a tenure track (Florida International University, 2014).The 9-month equivalent salary for all of faculty was $\$ 94,026$. For the fall semester 2014, the student/faculty ratio was 25.9:1. 


\section{FIU's Global Learning Professional Development Workshops}

The Office of Global Learning Initiatives (OGLI) conducts professional

development workshops to advance interdisciplinary, problem-centered learning in FIU's global learning (GL) courses and activities. Workshops are for faculty who are developing or revising courses for GL designation and for faculty teaching GLdesignated courses. Participants explore FIU's global learning outcomes, authentic assessments, active learning strategies, and multi-perspective content that can be implemented almost immediately in courses and activities across the curriculum and cocurriculum. Stipends are paid to faculty teaching GL designated courses for attending the workshop and completing a GL course assessment matrix as. Faculty members developing or revising a GL course also receive a stipend for attending the workshop and completing a GL course proposal. These proposals are developed and drafted by the faculty member with the help of OGLI staff and submitted to the Faculty Senate Global Learning Curriculum Oversight Committee (Florida International University, 2014a).

All workshop participants receive a flashdrive with documents used in the global learning course and activity development process. The full content of the flashdrive is available to current FIU faculty and staff through a website link. The core documents are a GL Foundations Course Approval Checklist (see Appendix C), a Discipline-Specific GL Course Approval Checklist (see Appendix D), a Model GL Syllabus (Annotated; see Appendix E), a Model GL Course Outcome Assessment Matrix (Annotated; see Appendix F), and FIU GL Rubrics -Global Awareness and Global Perspective (see Appendix G; Florida International University, 2014a). 


\section{Population}

The population for this study was FIU faculty members who had taken a global learning professional development workshop that is the precursor to the actual teaching of a global learning course who were thus eligible to be selected to participate in this study. A list of all faculty members who have taken a global learning professional development workshop is maintained by the Office of Global Learning Initiatives at FIU. There were 422 participants who had attended these workshops by the end of 2014. The types of professors included in the population included instructors, associate professors, adjunct professors, clinical professors, visiting professors, assistant professors and full professors (Florida International University, 2014b).

\section{Quantitative Stage}

This stage acted as a process to identify the key informants necessary for the appropriate collection of data from surveys and interviews to be used in the thematic analysis conducted in the qualitative stage of this case study.

\section{Sampling strategy}

The sample in the quantitative stage of this study consisted of all faculty members in the population who were contacted by email and consented to taking an online survey. The researcher attempted to contact everyone in the population. Selecting participants from the list of faculty who attended a global learning professional development workshop essentially created a purposeful sample because these faculty members have met the criteria of exposure to global learning. Simple random sampling was not needed because through the use of email the researcher contacted all members of the population simultaneously about study participation. Yin (2004) argued that researchers must 
demonstrate a good case selection technique in order to have viable cases. In this study, an expert and mentor reviewed the survey instrument (see Appendix A) used in order to select viable cases.

\section{Data collection}

Research questions were answered by using a survey developed by the researcher through consultation with his dissertation committee and experts in global learning and approved by the Institutional Review Board (IRB) of Florida International University. All faculty members at FIU who have attended a global learning professional development workshop were invited to take this survey to identify faculty members who have had a transformational learning experience related to the global learning professional development workshops. Data collection was done in the quantitative stage through the survey. The data collection procedure was to contact with an email letter (see Appendix H) all faculty members listed as having attended a global learning professional development workshop and use the email letter to inform the faculty members of the study and the hyperlink to a website where the faculty members could take the survey online (see Appendix H). Those participating faculty members answered the consent form (see Appendix I) online, partook of the survey, and were asked if they would like to participate in a follow up semi-structured interview (see Appendix B) for the qualitative stage of data collection. Hard copies of the consent forms for the survey were made available to all participants who wish to use them. Participants in the follow up interview had to sign a hard copy of a consent form.

Actions conducted to improve response rates followed Dillman's (2000) tailored design method for administering surveys and included reminder emails at steady 
intervals. After the survey was initially administered, the researcher emailed potential respondents a reminder at 15 day intervals. This pattern of reminder emails at 15 day interval points continued until the researcher reached the fifth 15 day interval point. The researcher reached the fifth interval point with a sample size of 97 survey participants.

After data collection, quantitative data from the modified online survey were downloaded into a Microsoft Excel spreadsheet and later a Microsoft Word document in order to make data analysis manageable

\section{Instrument}

The survey was put online using Qualtrics (@) software. The instrument had four sections (See Appendix A). The first section was an online consent form that the respondent had to acknowledge in order to proceed to the survey questions. The next section of the survey consisted of questions related to determining if a transformational learning experience occurred for university faculty in a global learning professional development workshop. This section had several yes or no questions that when answered in the affirmative was linked to a prompt that asked for a description of that answer. When a respondent answered in the negative to the yes or no question, the respondent was automatically sent to the next yes or no question and did not see the corresponding prompt asking for a more detailed explanation of a positive answer. Each yes or no question and its corresponding prompt for more detailed data were based on phases in Mezirow’s (1978; 1991; 1996) transformational learning theory founded on the themes of experience, critical reflection, rational discourse, and action. The questions in this section helped identify if a person had an incidence of transformational learning related to the global learning professional development workshops. 
The third section of the survey consisted of a set of demographic questions. Demographic questions were based on characteristics of university faculty. The demographics section served to verify that the respondents were FIU faculty, that they taught at least one global learning course, and to make evident any troubling pattern in the nature of who was making the responses. This section asked the respondents to describe their ethnicity, race, and what college or school within FIU they taught. Demographic questions were necessary because the list was used to determine the sample (Florida International University, 2014). Global learning demands that an individual's perspectives and the perspectives of others are explored (Florida International University, 2010) by faculty teaching students. The demographics of faculty identity may inform a faculty member's perspective and consequently their transformational learning and instruction.

This section of the survey also asked the respondent the number of global learning courses they teach, how often they teach a global learning course, and for how long they have been teaching a global learning course. These questions were necessary because not everyone who has taken a global learning professional development workshop has taught a global learning course. This was also important because this dissertation explored practice. In the global learning and internationalization literature, there is anecdotal evidence that global learning is more challenging to implement in some disciplines (Duerst-Lahti, 2005; Takacs, 2008) than others that are considered more global (Barbiero, 2008; Perry \& Mander, 2005)..

The final section of the survey was an invitation for the respondent to participate in a follow up semi-structured interview in the next phase of the research. The Qualtrics 
(@) software assigned each participant a numeric identity code that helped maintain the anonymity of the participant.

\section{Data analysis}

Data analysis in the quantitative stage was done primarily through the scoring of the second section of the survey with a researcher developed categorical index created through expert and mentor review. This was followed by a basic statistical analysis to determine frequency.

\section{Categorical index}

The categorical index allowed the researcher to classify all surveys into the categories of incomplete, non-transformational learning, partial learning, and transformational learning. The second section of the survey was made up of a series of yes or no questions that when answered in the affirmative lead to a descriptive prompt where the respondent must describe their answer. A positive response in a yes or no question was necessarily supported by a descriptive response in the corresponding prompt for that question. The respondent could not for example leave the descriptive prompt blank or write something that did not have to do with the focus of the corresponding yes or no question. An affirmative statement on a yes or no question that was not supported by a response in the corresponding descriptive prompt was considered a negative statement. Supportive responses to a yes or no question were needed to make clear that transformational learning was related to the global learning workshops.

The questions in the second section of the survey were based on the phases and themes that lead to transformation. The theme of experience was embedded in all the questions since all the questions were worded in way that explore the experience of 
global learning in the professional development workshop as a possible disorienting dilemma for faculty members. The theme of action was also embedded in some of the questions. The researcher looked for responses in all the descriptive prompts answered in each survey that correspond with any of the phases in the themes of critical reflection, rational discourse, and the need for action that are part of transformational learning theory (Mezirow, 1978, 1991, 1996).

Critical reflection on an experience for example can lead to phases of selfexamination with feelings of guilt or shame and/or a critical assessment of assumptions. In rational discourse, an individual can have a recognition that one's discontent and the process of transformation are shared and that others have negotiated a similar change. An individual begins an exploration of options for new roles, relationships and actions. The phases in the theme of action can include a provisional trying of new roles; a planning of a course of action; acquisition of knowledge and skills for implementing one's plans; a building of competence and self-confidence in new roles and relationships; and finally a reintegration into one's life on the basis of conditions dictated by one's new perspective (Mezirow, 1978, 1991, 1996).

In order for the experience to be considered transformational, the respondent must first answer in the affirmative to at least one of the yes or no questions and describe their answers in the corresponding prompt for each yes or no question answered in the affirmative. According to transformational learning theory, critical reflection on an experience must be followed by rational discourse. Because all questions in the second section of survey are based on the experience of global learning in a professional development workshop, the data from the survey must describe at least one phase of 
critical reflection, at least one phase of rational discourse, and at least one phase of action in order for the survey to be categorized as having transformational learning. This is necessary because critical reflection by itself does not lead to transformation. It could lead to simple verification of old assumptions. Rationale discourse and action needs to be supported by critical reflection because many actions that seem to be based on rationale discourse can be stimulated by other forces that are not based on transformation. For example, a person may change their syllabus or move from a lecturer role because they were told to do so and not because of a change in the way they perceive learning.

For this dissertation, a survey in which the respondent had not answered any questions other than giving consent was considered incomplete. A survey in which the respondent answered in the negative to all yes or no questions was considered nontransformational learning. A survey in which a respondent only showed instances of either critical reflection or rational discourse or action was considered partial learning. A survey in which a respondent showed instances of critical reflection, rational discourse, and of action was considered transformational learning. Only the surveys that showed transformational learning were considered for the qualitative stage of the dissertation.

\section{Quantitative analysis}

In this study, the survey used the second section to screen for sources of information for the qualitative part of the case study and to answer the first research question. Do university faculty members who have attended global learning professional development workshops report a transformational learning experience? Once all surveys were classified with the categorical index by the researcher, the researcher determined the frequency at which a respondent's survey answers fit the category of incomplete, non- 
transformational learning, partial learning, and transformational learning. In order for the first research question to be answered in the negative, the frequency at which a survey indicated transformational learning had to less than one. In order to answer the first research question in the affirmative, the frequency at which a survey was categorized as transformational learning had to be one or more. If the statistical analysis supported the conclusion that the first research question was in the affirmative, the dissertation proceeded to the qualitative stage.

\section{Qualitative Stage}

This qualitative stage built on the screening process created in the quantitative stage to conduct multiple interviews.

\section{Sampling strategy}

For the qualitative stage of this study, the sample was selected from a pool of all faculty members who had completed the survey, whose responses indicated transformational learning may have occurred in or through the workshops, and who consented to participate in a semi-structured interview. The faculty members who had experienced transformational learning related to global learning and who agreed to an interview were placed into a pool of potential interviewees.

Of the 42 surveys labeled as transformational learning, only 27 participants agreed to a follow up interview on their survey. Of these 27 participants, only 23 surveys indicated that a global learning class had been taught. This meant that only 23 participants could fully answer all the research questions. The researcher ranked these surveys based on the level of richness of data in their textual responses from the most to least. The researcher initially selected 15 participants to interview from this pool with 
the most richness of detail expressed in the words and ideas the participants typed in their survey text.

The participants were selected based on the richness of the data they provided in their survey responses and perceived depth of their transformation based on that data. For example, a respondent who had answered yes to several questions in the survey and provided lengthy text responses that described their answers was given precedent over a respondent who answered yes to fewer questions and gave fewer descriptions of their answers.

Because of the lack of responses to interview requests, the researcher sent a reminder interview request to the initial 15 participants who had not responded after two weeks. The researcher then contacted all the remaining faculty members in the pool and later also sent a reminder email to those faculty members who had not responded after two weeks. In total, five faculty members responded to interview requests from the researcher. According to Creswell (1998), five participants is the smallest sample that is acceptable. Romney, Batchelder, and Weller (1986) argued that samples as small as four individuals can yield highly accurate information. In this study, no new themes emerged in the last interview.

\section{Instrument}

Research questions were answered by using a semi-structured interview guide (See Appendix B) developed by the researcher through consultation with his dissertation committee and experts in global learning and approved by the Institutional Review Board (IRB) of Florida International University (FIU). All faculty members at FIU who had attended a global learning professional development workshop, had taken the survey, 
consented to an interview (See Appendix J), and were identified as having had a transformational experience due to the global learning workshop were invited to be interviewed. The interview guide had several open questions and prompts about transformational learning and the global learning professional development workshops. Yin (2004) argued for the development of a case study protocol especially in describing the use of questions and how they are administered. Mentor and expert reviews helped in the development of this protocol.

\section{Data collection}

The procedure for the qualitative stage of data collection was the following: The interviews were scheduled at a time and location of the faculty member's convenience. The researcher conducted the interview. Each interview was completed within 30 to 60 minutes. The interview was recorded with a digital audio recording device. A second audio recording device was used as a back up. The researcher also took notes. Identities of those being interviewed was kept confidential. The interviewer used questions to go into greater depth about transformational learning and how it has informed the teaching of the faculty member. After the interview, the researcher transcribed the data and emailed it to the interviewee as a member checking moment. From the surveys, the researcher analyzed the data from the questions in the second section of the survey. After that analysis, interviews continued to be scheduled and conducted until the sample of available interviewees was exhausted.

\section{Data analysis}

Yin (2004) suggested that a researcher must decide on a way to analyze the data. In this study, data was analyzed for themes within the case study of the global learning 
professional development workshops. In the qualitative analysis section of this study, the data from the surveys was analyzed first. Then the data from the interview was analyzed. Then data from both sources were compared. The qualitative data collected from the survey and semi-structured interviews were eventually used to answer the second research question through a process of thematic analysis: How do university faculty members who have attended global learning professional development workshops perceive global learning curricula as transformational learning?

The data to answer this second research question can be found in the responses to the open descriptive prompts in the survey instrument (see Appendix A) and in the transcribed responses to the semi-structured interviews (see Appendix B). The third main research question is: How do university faculty members who have attended a global learning professional development workshop and report having had a resulting transformational learning experience apply this transformation to their teaching? The data to answer this third main research question was also answered with the same process used for the second research question.

\section{Thematic analysis}

Thematic analysis is a method for identifying, analyzing and reporting patterns (themes) within data. A theme is a pattern found in the information that describes and organizes observations (Boyatzis, 1998). This dissertation used an inductive approach to analyze themes. An inductive approach means the themes identified are strongly linked to the data themselves (Patton, 1990), Semantic (or manifest level) themes and latent themes are the two kinds of themes that can be found. With a semantic approach, the themes are identified within the explicit or surface meanings of the data. These themes are directly 
observable. In a latent approach, the researcher tries to determine a theme based on what is behind what is being explicitly communicated. They are an underlying cause of a phenomenon (Boyatzis, 1998).

The researcher of this dissertation used the Boyatzis's (1998) method of thematic analysis to review the data collected from the semi-structured interviews because of the detailed approach it entailed in the developing of a code set. The researcher also used thematic analysis on the text data collected in the surveys that were categorized as transformational learning by the researcher.

In Boyatzis’s (1998) method of thematic analysis, the researcher reads and rereads the data to develop themes by reducing the raw information in the data. This can be done by summarizing the data. One starts with a piece of data and begins to identify a pattern by comparing the old data to newer data. The unit of analyses for themes are words, phrases, and/or sentences that together promote an idea. The researcher takes note of what is semantically or explicitly communicated by the data and also what is latently communicated in the data. Once the pattern of a theme is identified it is named. It is then defined, factors that help flag the occurrence of this theme are listed, qualifications and exclusions to the theme are described, and positive and negative examples of it are listed by the researcher in order to be able to identify it when it occurs in the readings again. The theme thus becomes a code that can be compared to new incoming data as the researcher continues to explore the data. A group of codes become a code set. The researcher uses the code set he develops to go over the data again and to mark off the data that fits certain themes. A final code set is created, used, and validated as the researcher goes over the data completely and checks to see if a theme or code can be collapsed into 
another for greater clarity. The researcher also compares the code set to the research questions it answers in order to determine if further clarification or work is needed (Boyatzis, 1998).

Yin (2004) suggested that a researcher must decide on a way to report the data. The data from the survey is reported first. Then the data from the interviews is reported. The data is reported through the use of sub-themes from the code set and vignettes of examples of quoted texts from the participants. The quotes from the respondents to the survey used a numeric code assigned to each survey respondent by the Qualtrics(@) survey software used to administer the survey. These codes assigned to each survey respondent were relabeled with a simpler number scheme. Faculty members being interviewed were assigned pseudonyms when quotes were used. The sub-themes from the survey and the interview were later compared and reported as larger themes. Later in the dissertation, the researcher used the sequence of research questions to organize the reporting of the data in the discussion of the findings.

\section{Integrity Measures}

Several strategies were used in this dissertation to help develop consistency in the research process. The researcher employed member checking in the qualitative stage of data analysis. Member checking is a process of sharing data and preliminary interpretations with the participants from whom the data were obtained (Merriam, 2002). The researcher first did this by transcribing the data from the semi-structured interview and emailing it to the participant who gave the interview for them to give any feedback that can improve the accuracy of the data. 
These faculty members were given a week to respond. There was agreement between the faculty members and the researcher on the content of the transcripts. Minor changes on some transcripts were made based on grammar. The researcher also sent the preliminary findings of the study to the faculty members. Faculty members were shown how their particular contributions fit into the sub-themes and themes developed by the researcher and how these would be used to answer the research questions. The faculty members again agreed with the content of the findings. Some made minor changes in wording. A faculty member questioned the way their speech pattern was presented in a particular passage. The researcher allowed the participant to hear the audio recording of this passage which showed the speech pattern to be correctly transcribed. The researcher and this participant came to agreement on this issue. Another respondent explained a particular part of their data with more precision. All feedback from the respondents that were interviewed were incorporated back into the study.

An audit trail is a collection of researcher notes. It contains detailed information about the methods, procedures, and decisions made during the course of the research (Merriam, 2002). A researcher journal made electronically in a word document was used to establish an audit trail that contains information about all of the activities related to the project in order to be able to account for any of the decisions made during the course of this project (Patton, 2002). The audit trail allows the researcher to share information with others about the process in order to gain feedback while also allowing the researcher to be transparent in their work especially with the use of peer, expert, and mentor review.

Mentor and expert review were used as the process and details were shared with my major professor, committee members, and experts in global learning and 
transformational learning. The researcher for example used part of the audit trail to discuss the way surveys were being categorized. The audit trail also allowed the researcher to explain the member checking process.

\section{Data Management}

All data for this study was saved on flashdrives or folders and kept in a locked desk only the researcher can access. The data for this study will be kept for three years following the completion of this study.

\section{Summary}

This chapter outlined the method used in this dissertation. The purpose, research questions, research design, and population of this study were described. Then the sampling, instruments, data collection, and data analysis for the quantitative and qualitative stages of this study were described. Lastly, integrity measures and data management procedures were described.

\section{Overview of Succeeding Chapter}

In the next chapter, the quantitative findings of this study will be presented. 


\section{CHAPTER IV}

\section{FINDINGS: QUANTITATIVE STAGE}

In this chapter the researcher reports the quantitative findings. These findings are comprised of the results of the survey that was administered to faculty members who had done global learning professional development workshops at FIU and had agreed to participate in this study.

Table 1

Transformational Learning Survey Results

\begin{tabular}{ccccc} 
Incomplete & $\begin{array}{c}\text { Non- } \\
\text { Transformational } \\
\text { Learning }\end{array}$ & Partial Learning & $\begin{array}{c}\text { Transformational } \\
\text { Learning }\end{array}$ & Total \\
\hline 17 & 12 & 26 & 42 & 97
\end{tabular}

There were a total of 97 respondents to the survey (See Table 1). The researcher labeled the surveys as either incomplete, non-transformational learning, partial learning, or transformational learning. There were 17 surveys that were labeled Incomplete. There were 12 surveys that were labeled as Non-Transformational Learning. There were 26 surveys that were labeled as Partial Learning. There were 42 surveys that were labeled as Transformational Learning. A narrative that emerges from the quantitative data is that many of the faculty members whose surveys were labeled as Transformational Learning come from similar personal and academic backgrounds. This narrative and the responses to the global learning professional developments are explored in this chapter for the surveys that were categorized as transformational learning. Demographic detail for surveys categorized as non-transformational learning can be found in Appendix K. 
Demographic detail for the surveys categorized as Partial Learning can be found in Appendix L. A brief overview of the demographic data of the non-transformational learning and partial learning surveys reveals that faculty in those surveys had similar characteristics to the faculty whose surveys were categorized as transformational learning. Since surveys that were labeled incomplete by definition lack data, it is impossible to make any inferences about the characteristics of faculty members who created them.

\section{Quantitative Findings}

According to the findings of the quantitative stage, several university faculty members who have attended global learning professional development workshops report a resulting transformational learning experience. Since the research focused on transformational learning, the following findings are based on all surveys completed by university faculty members that were subsequently labeled as transformational learning.

Certain characteristics become clear about the profiles of faculty members whose surveys were categorized as transformational learning. These characteristics can add context to their responses to transformational learning related survey questions. These characteristics are evidenced by responses to questions 22 through 29 of the survey that deal with demographic questions. First, many faculty members reported their ethnicity as being White (See Table 2). The 25th question or prompt asked on the survey was: What is your ethnicity? Answers to this question varied. Some respondents fit more than one category. Some respondents were so specific it was necessary to report their ethnic identity in less specific terms in order to keep their identities confidential. 
Table 2

Ethnicity of faculty members as reported in surveys

\begin{tabular}{|c|c|}
\hline Ethnicity & Responses \\
\hline African & 2 \\
\hline African American & 2 \\
\hline Asian & 3 \\
\hline Asian American & 1 \\
\hline Caucasian & 3 \\
\hline Caribbean & 4 \\
\hline European American & 3 \\
\hline Hispanic & 2 \\
\hline Jewish & 5 \\
\hline Latin Hispanic & 3 \\
\hline Middle Eastern & 1 \\
\hline Mixed & 1 \\
\hline White & 6 \\
\hline White European & 5 \\
\hline White Hispanic & 3 \\
\hline White Latino & 2 \\
\hline White Non Hispanic & 2 \\
\hline Chose not to answer & 2 \\
\hline
\end{tabular}

A second characteristic that revealed itself in the findings was that many faculty members reported their race as also being White (See Table 3). The majority of the 
faculty members were White.. The 26th question on the survey was: What is your race? Please select.

Of the six participants who listed their race as Other, two participants described their race as Mixed, one as Brown, and one as White Hispanic. Two participants stated that they chose not to give this information.

Table 3

Race of faculty members as reported in surveys

\begin{tabular}{cc}
\hline Race & Responses \\
\hline White (Non-Hispanic) & 22 \\
Black & 4 \\
Hispanic & 6 \\
Asian or Pacific Islander & 4 \\
Other (Please specify) & 6 \\
\hline Total & 42 \\
\hline
\end{tabular}

A third characteristic of the faculty members that emerged was that many of them worked in the College of Arts and Sciences. The 27th question or prompt asked on the survey was: In which FIU school or college do you teach?

Of the four respondents who chose other in Table 4 there were three participants who described their answer as Stempel College of Public Health and Social Work. There was one participant who described their answer as "No longer at FIU.” There were no participants who described their answer as "Choosing not to give this information." 
Table 4

FIU School or College of Faculty Members as Reported in Surveys

\begin{tabular}{|c|c|}
\hline School or College ‘ & Responses \\
\hline College of Architecture and Arts & 6 \\
\hline College of Arts and Sciences & 15 \\
\hline College of Business & 1 \\
\hline College of Education & 4 \\
\hline College of Engineering and Computing & 3 \\
\hline Honors College & 1 \\
\hline College of Law & 0 \\
\hline Herbert Wertheim College of Medicine & 1 \\
\hline Nicole Wertheim College of Nursing and Health Sciences & 2 \\
\hline Chaplin School of Hospitality and Tourism Management & 0 \\
\hline School of Journalism and Mass Communications & 3 \\
\hline Other: & 4 \\
\hline Total & 42 \\
\hline
\end{tabular}

Another important characteristic about these faculty members is that the majority of them taught at least one global learning course. The 22nd question or prompt asked on the survey was: How many global learning courses do you teach? 
Table 5

Number of global learning courses taught by faculty members as reported in surveys.

\begin{tabular}{cc}
\hline Global Learning Courses. & Responses \\
\hline None & 4 \\
One courses & 22 \\
One to two courses & 6 \\
Two to three courses & 5 \\
Three to four courses & 1 \\
Five courses & 1 \\
One or more in the past & 3 \\
\hline
\end{tabular}

The majority of these faculty members teach a global learning course every semester. The 23rd question or prompt asked on the survey was: How often do you teach a global learning course? Answers to this question varied as seen in Table 6.

Table 6

How often faculty members teach a global learning class as reported in surveys.

\begin{tabular}{cc}
\hline Global Learning Courses & Responses \\
\hline Every semester & 22 \\
One semester in a year & 8 \\
Two semesters in a year & 7 \\
Every semester in the past & 1 \\
Every few years & 1 \\
Never & 1 \\
Not applicable & 1 \\
\hline
\end{tabular}


The majority of these faculty members have been teaching global learning courses for a long time. Please refer to Table 6. The 24th question or prompt asked on the survey was: How long have you been teaching a global learning course?

\section{Table 7}

How long faculty members have taught global learning courses as reported in surveys.

\begin{tabular}{cc}
\hline Global Learning Courses & Responses \\
\hline Never & 3 \\
Not Applicable & 0 \\
Beginning of global learning initiative & 5 \\
One year or less & 7 \\
Two years & 8 \\
Three years & 7 \\
Four years & 6 \\
Five years & 4 \\
Six to eight years or more & 2 \\
\hline
\end{tabular}

The majority of these faculty members initially agreed to participate in a future interview. The $28^{\text {th }}$ and 29 th question or prompt asked on the survey was designed to invite participants to a follow up semi-structured interview. There were 27 participants who agreed to an interview.

The following passages discuss the responses to questions two through 21 on the surveys. Question one is not shown since it is the consent to participate. The third, fifth, seventh, ninth, 11th, 13th, 15th, 17th, 19th, and 21st items are descriptive prompts that require a textual response be typed in by the survey respondent in order to proceed through the rest of the survey. These prompts were only shown to survey respondents if they answered yes to a previous yes or no question. Responses varied in length and nature 
and these responses are considered part of the qualitative research described in the next chapter.

Table 8 below shows the numbers of responses to the transformational learning related items on the global learning survey. These responses to the global learning surveys categorized as transformational learning by the researcher paint a picture of these FIU faculty members. Reactions to the transformational learning questions reveal that many of these faculty members reconsidered the way they taught and the content of their classes. Forty out of 42 respondents reconsidered the way they taught. Twenty-seven out of 42 respondents reconsidered the content of their classes. 


\section{Table 8}

Number of responses to question/prompt(s) two through 21 on the global learning survey.

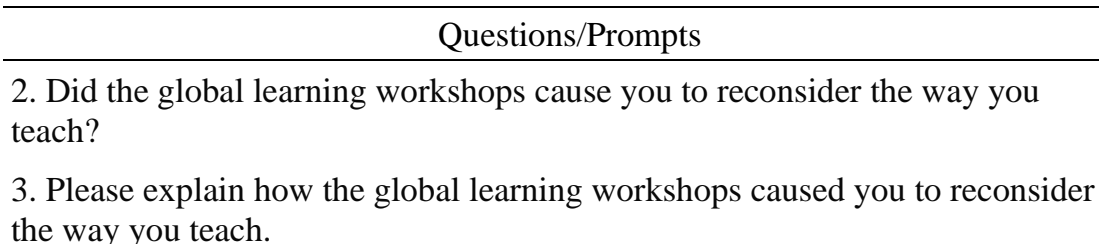

4. Did the global learning workshops cause you to reconsider the content of your courses?

Yes

No Text

5. Please explain how the global learning workshops caused you to reconsider the content of your courses.

40

2

6. Did the global learning workshops cause you to reconsider your relationship with your students? Yes or no.

7. Please explain how the global learning workshops caused you to reconsider your relationship with your students.

8. Did the global learning workshops cause you to reconsider your views on learning?

9. Please explain how the global learning workshops caused you to reconsider your views on learning.

10. Did you learn something due to the global learning workshops that made you feel uneasy?

11. Please explain what you learned due to the global learning workshops that made you feel uneasy.

12. Due to the global learning workshops, did you experience something that contradicted a previously held belief?

13. Please describe the experience that contradicted a previously held belief.

14. Did you have an epiphany due to the global learning workshops?

15. Please describe this epiphany due to the global learning workshops.

16. Did you recognize a shared process of transformation shared by you and others due to the global learning workshops?

17. Please describe this shared process of transformation shared by you and others due to the global learning workshops.

18. Did you develop new relationships due to the global learning workshops?

19. Please describe the nature of these new relationships due to the global learning workshops.

20. Was there a change in your self confidence due to the global learning workshops? 
Another important finding is that the majority of these faculty members were inspired to reconsider their relationships with students and their views on learning. Twenty-three out of 42 respondents reconsidered their relationships with students. Twenty-two out of 42 respondents reconsidered their views on learning. Many faculty members did not report in their surveys feeling uneasy about something they learned or having to grapple with a contradiction. Eleven out of 42 respondents felt uneasy about something thing they learned. Six out of 42 respondents grappled with a contradiction.

Many faculty members reported a shared sense of transformation with others but not all faculty members reported an epiphany. Twenty-two out of 42 respondents reported a shared sense of transformation. Eighteen out of 42 respondents reported an epiphany. For many faculty members, the global learning professional development workshops led to new relationships and for a few faculty members a change in selfconfidence. Twenty-six out of 42 respondents developed new relationships. Fifteen out of 42 respondents felt a change in self-confidence.

\section{Summary}

This chapter presented the quantitative findings for this research. The findings reveal that there were 42 faculty members whose surveys could be classified as having had transformational learning. Many of the faculty members with surveys that were classified as transformational learning identified themselves as being ethnically and racially White. Many of these faculty members are very familiar with the teaching of global learning courses. The majority of these faculty members work in FIU's College of Arts and Sciences. The majority of the faculty members reported a reconsideration of the way they teach, the contents of their courses, their relationships to students and other 
faculty members, and their views on learning. Many of them also agreed to a follow up interview. These findings set the foundation for the qualitative analysis of data.

\section{Overview of Succeeding Chapter}

In the next chapter the qualitative findings of this study will be presented. 


\section{CHAPTER V}

\section{FINDINGS: QUANTITATIVE STAGE}

This chapter presents the qualitative findings of this study. Each qualitative research question is answered through the use thematic analysis of text responses to the survey and the transcripts of the interviews. The researcher took surveys that showed transformational learning and conducted interviews with faculty members who had completed those surveys. Themes were then developed from the transcriptions of those interviews. By using coding and analysis, these themes and the data from which they emanated were reviewed several times until these themes were modified, compared and refined into larger themes by the researcher. These larger themes were used to develop a code set that was used by the researcher to answer the second and third research questions of this dissertation.

The researcher presents the findings in this chapter in the following way: the particular research question being explored is stated and then the researcher presents the major themes and sub-themes that correspond to or emanate from the answers to that question. Excerpts from the codebook developed by the researcher using Boyatzis procedures for thematic analysis are used to define each theme, give indicators of the theme, give an example of the theme, and describe the limits of the theme. In the discussions of the examples of each theme, sub-themes may be referred to. Codes for these sub-themes were also compiled by the researcher but are not represented in this dissertation for the sake of brevity and clarity.

This is followed by excerpts from the survey data and interview transcripts related to that particular theme that serve as vignettes to give an in-depth understanding of the 
theme to the reader. For the survey excerpts, a numeric code assigned by Qualtrics @ software to each survey was replaced by a numeric code assigned by the researcher that ranged from one to 42 . The code identified the survey respondent with a number, followed by a comma and a number that indicates from which survey page the quote comes from, followed by a forward slash and line numbers indicating where in the survey the quote can be found. An example would be a quote cited (Respondent 1, 2/2) indicating that the quoted data comes from the survey of respondent 1 and can be found in on page two on the second line of the transcript for that particular survey. Respondents to the global learning survey actually typed in their responses themselves and this allowed them to purposely use language styles and literacies to express themselves. This could manifest itself in the complete capitalization of key words, the unorthodox or aggressive use of dashes, slashes, and punctuations marks, unique or grammatically incorrect spelling and/or grammar, the inserting of symbols, and other details. Respondent 4 for example used the capitalization of key words when describing the preparation of students for the future when he or she types "Students need to be consciously and critically looking far into the FUTURE, and deeper into GLOBALIZATION...” (Respondent: 4, 2/3-5).

The researcher of this study presents the text of the survey responses as they were written and has made no changes to them other than editing them for length or eliminating details that may identify the respondent. For quoted excerpts from the interview transcripts, each respondent is identified by a pseudonym, followed by a comma, a page number, a forward slash and a line number. For example a quote cited (Helen, 5/24-27) identifies the author of the quote as Helen and this data can be found on 
page five of Helen's interview transcript in lines 24-27. For both the presentation of the data that comes from the surveys and the data that come from the interviews, an ellipsis will often show where the researcher has edited out a section of the text for length or content that identifies the person typing or speaking. A variation of the words "omitted by researcher” may also appear in brackets to show where the researcher has eliminated or changed words that could identify the university faculty member who created the statement.

\section{Research Question Two}

Research question two asked: How do university faculty members who have attended global learning professional development workshops and who reported transformational learning perceive global learning curricula as transformational learning?

\section{Qualitative Findings for Research Question Two}

The findings show that FIU faculty who attended a global learning professional development workshop and who reported transformational learning perceived this transformation as a series of individual and collective opportunities and challenges. The theme of individual opportunities included the sub-themes of revelation, clarity, negative transformation, and empowerment. The second theme is individual challenges. The theme of collective opportunities included the sub-themes of positioning, multidisciplinary communities and enthusiasm. The theme of collective challenges included the sub-themes of global and a call to leadership. The following table identifies the major themes and sub-themes as they relate to research question two. 
Table 9

Major themes and subthemes in response to research question two.

Major theme

Individual opportunities

Collective opportunities

Individual challenges

Collective challenges

\section{Sub-themes}

\section{Revelation}

Clarity

Negative transformation

Empowerment.

Positioning

Multi-disciplinary communities

Enthusiasm

Varied

Global

Call to leadership

The following passages describe the major themes and examples of them as they relate to research question two.

\section{Individual Opportunities}

The first way that transformational learning for university faculty members who have attended global learning professional development workshops can be shown is in the form of one or more individual opportunities. Individual opportunities are defined as intrinsic opportunities that the respondents describe that manifest themselves from the learning and understanding afforded by the global learning professional development workshops. Indicators of this theme occur when the respondent uses words like I, 
understand, and new. Qualifications to this theme include the fact that these opportunities have to be related directly or indirectly in some way to the goals of the global learning professional development workshops. Exclusions to this theme are narrowly defined. Opportunities that are not related to the goals of the global learning workshops are excluded. For example, a statement where a university faculty member is tipped off to a business opportunity by another faculty member is not included in this theme.

Examples of the theme of individual opportunities occurred in various ways for university faculty members. An important opportunity came in their understanding of global learning. For some faculty members the workshop was something they experienced as a revelation or clarification of concepts. The sub-theme of revelation occurred when global learning or some facet of the global learning workshop was experienced as something new that the respondent had little or no previous knowledge about. An example of the sub-theme of revelation showed in this statement by Melissa: "I had no idea what global learning was. I was just basically doing the study abroad class. The course we were using to create a study abroad class was a global learning class already. It would have been anyway. Obviously I think any study abroad class would be a global learning anyway.” (Melissa, 1/33-36) Dan also found global learning to be a revelation: "Global learning as a concept was relatively new to me so it was very useful for me you know in the workshop to see that particular perspective on things. So yeah, I found it very useful, very helpful on the developing of the syllabus that we worked on.” (Dan, 1/45 -2/2).

While the term global learning was known to many respondents what it actually 
meant was a mystery as the statement given by Melissa illustrates:

“The course I was going to be teaching had a global learning designation. We used an existing course to create a special section. I had to go through this because I was now teaching a global learning designated class. And I said what the heck is global learning? So I went to the workshop. It was very good.” (Melissa, 1/38-41)

For Dan, aspects of what was taught at the global learning professional development workshops were as revealing as the larger concepts. In the following statement, Dan describes a solution he learned about to deal with the problems of group learning:

"I would get complaints from students you know who said they were in a group but they were doing all the work and nobody else did anything. Of course, my experiences had been very similar. But I didn’t know how to address that. How do you deal with that. Well one thing the workshop has dealt with was this exact same topic. The approach that was suggested is that the students evaluated themselves. Peer evaluations of team mates. Wow. That was like a revelation. That is pretty cool. We have been doing that in the (global learning) class ever since.” (Dan, 3/24-31)

Survey respondent 23 wrote that what was revealed at the workshops "Made me think more globally and critically” (Respondent: 23, 1/20).

Another survey respondent added "Well, small epiphanies! The workshop on teams was quite revealing. The importance of active learning really hit home (Respondent: 3, 3, 3-4). 
The sub-theme theme of clarity occurred when some facet of the global learning workshop led to a respondent feeling that an issue that was once understood in an incomplete or abstract way is now understood in a moment of better clarity for the respondent. An example of the sub-theme of clarity is described by Dan:

"I think I had a sort of fuzzy notion of the importance of a global perspective before the workshop but it was very amorphous and kind of ill formed. I knew that you know that global knowledge and understanding, global environment was important but not really in as a systematic, well thought out way as global learning deals with it. And so I wouldn’t say I was resistant to it exactly but I think what it did was it sorted out my thinking on the matter. It was very helpful to get a sort of systematic approach to global learning perspective that you get from the global learning perspective. So for me that was the most intellectual stimulating, interesting part of the workshop. And so we went through several readings, some book learning and several thinkers and I think it helped to clarify my frame work on just what global learning is.” (Dan, 4/18-27)

Kelly also describes how the workshops brought clarity to the elements of global learning:

"The workshop actually helped in the delineation of all the three aspects of global learning. As I make it a point to tell the students how different these three aspects of the global learning is. One is global awareness where you just come to know about one aspect of the one culture, the one idea, the one concept, is the awareness from the different parts of the globe. But the perspective, once you have the awareness of all these different elements then only can you develop the 
perspective. So this is one thing, this is one important idea that came from my attending the global learning workshop.” (Kelly, 2/35-41)

Respondent 18 explained that "It helps me understand the distinction between awareness and perspective, which I am able to impart with students. It also helped me to convey the value of cultural competency to students” (Respondent: 18, 1/17-18).

Coming to an understanding of what global learning means at the global learning professional development workshops did not always lead to a positive reaction from university faculty members. Individual opportunities to state grievances appeared. Some faculty members experienced what can be called a negative transformation. The subtheme of negative transformation occurs when the respondent so negatively views what they learned at the global learning professional development workshops that it sets off a protest that shows critical thought, discourse, and/or action. An example of the sub-theme of negative transformation occurs with this statement by Frank: "The GL workshop was basically an introduction to the concept of global learning and I don't know how to say this, an indoctrination into what the team there had intended”. (Frank, 1/19-21)

Respondent 13 wrote of the global learning professional development workshops: 1) the name is misleading - many of the courses are not global in the way I perceive the meaning of the word 2) Many classes that I already considered global were not grandfathered into the program 3) The Global Learning program seems to be pushing a western centric ideology where the western way is intrinsically considered superior (Respondent: 13, 2/15-17).

Some university faculty members disagreed with aspects of what they learned at the workshops. For example: 
The only real thing that makes me uncomfortable is maybe the different ideas about academic dishonesty from around the world. I want to be fair to ALL my students, but at the same time I want to maintain high standards in regards to academic honesty and plagiarism. (Respondent: 27, 2/25)

For respondent 6, the negative transformation was serious enough to make the respondent question aspects of their profession.

It made me realize how much trend and fashion governs the expectations about teaching. It made me determined to take time when considering the substance behind a new idea. Also, the request made in the workshop that I require students to pledge to use a global perspective in future seemed so unethical that it made me reflect on larger ethical questions in our profession....We were asked to include in our evaluation of students their pledge to view things from a global perspective. While I think that would be a good thing, it seemed unethical to grade students on their professed beliefs. (Respondent: 6, 1/21-24, 2/16-17)

University faculty members also felt that the workshops led to an opportunity to feel or renew a sense of positive emotional energy. One form of positive energy came through a feeling of empowerment. The sub-theme of empowerment occurred when the respondent feels that the global learning professional development workshops has led to them gaining a sense of confidence. The following are examples of the sub-theme of empowerment: "I increased my self-confidence significantly, somehow those workshops empowered me. Accepting the differences made accept myself as an enriching faculty at FIU. Besides I realized that I have many things to offer to FIU and my department.” (Respondent: 22, 4/ 3-5) 
Respondent 10 describes how this sense of empowerment influenced their relationship to students: "I became more sensitive, but also more curious and less reluctant to query students about their cultures” (Respondent: 10, 3/1)

Respondent 27 explains that "Making these connections and seeing other people's work has made me feel more confident” (Respondent: 27, 3/40). Respondent 10 explains the empowerment in a different way by simply stating that "Knowledge leads to confidence” (Respondent: 10, 3/31).

Respondent 21 gives an example of how empowerment can be complicated with this statement:

"both yes and no... confidence that I am now mentally equipped to offer a global learning course. No -- because the realization that the teaching style that I had honed over many years of practice -- is not really a good way to teach. So, you have to master a new way of teaching -- it takes a lot of time and effort ---and doubts on your ability. "(Respondent: 21, 3/ 32-34)

For respondent 22, empowerment meant dealing with internal and external obstacles as evidenced by this statement:

I met wonderful people, starting by the Global Learning leaders, they have been supportive and engaging. I discover that FIU have many interesting professors. However I have not developed professional links or multidisciplinary adventures. I think this is the next step. I am a little bit out of the loop due to the topics now addressed, they are mainly in the social and humanistic field. Living our reality or shortage of time I have been somehow selected other groups. But the Global 
learning certainly modified the foundations of my career at FIU. (Respondent: 22, 3/ 29-33)

Empowerment can drive engagement for university faculty members. Respondent 21 wrote "I have become more engaged through the discussion board and through comments and suggestions for the group and individual projects” (Respondent: 21, 1/3839).

\section{Collective Opportunities.}

A second way that transformational learning occurred for university faculty members who have attended global learning professional development workshops was in the form of one or more collective opportunities. Collective opportunities are defined as extrinsic opportunities the respondents describe that manifest themselves from social interactions afforded by the global learning professional development workshops. Indicators of this theme may occur when respondents may use words like opportunity, chance, gateway, bridge or synonyms related to these words. Qualifications to this theme involve several factors. Opportunities have to be related to the global learning professional development workshops, must come from social interactions or external factors, and must be significant. Exclusions are opportunities that are not related to the global learning professional developments workshops.

Examples of the theme of collective opportunities are varied and be experience in many ways. A collective opportunity presents itself in the sub-theme of positioning. The positioning sub-theme occurred when the experiences of university faculty inspires them to identify or position themselves on a spectrum in relation to other faculty members where one point of the spectrum are true believers and the other end of the spectrum are 
the people who do not believe in global learning as it is being promoted in the global learning professional development workshops. The following are examples of the subtheme of positioning:

Martin says of his belief in the global learning process “...I was bought in. I was in it in the beginning” (Martin, 4/30). Dan describes how he and other faculty members felt about the global learning professional development workshops in this way:

"Some of the faculty I think were nervous that this was a top down imposition from administration but it was not at all. It was much more you know your ideas are driving the development of the syllabi and they were there basically you know to get you thinking about different aspects of how might the course come together and also incorporating global learning components.” (Dan, 1/41-45)

Frank bluntly states how the collective opportunity to interact and discuss issues informed his thinking:

"My sense is that the people who conceptualized this they are among the true believers. These are the folks who are just certain that global is in everything. It is in our breakfast cereal. It is in every class we teach no matter what it is about. We could be talking exo planets and we are talking global. I don't think that is true. I think it is possible to over emphasize what global is. It becomes a sort of seven degrees of Kevin Bacon in order to get whatever your teaching back to global. The main challenge for me is there some reasonable, natural sensible connection between what I am teaching in this course and what is intended as global by this group?” (Frank, 1/26-33)

Frank explains further: 
"I would not say that I don't believe in global learning. Rather, I would say that I don't believe that "global" is as pervasive as the true believers seemed to suggest. I am fine with global learning, but I am not prepared to call everything global. There were and are significant issues, problems, trends, developments, subjects, etc., that are not global. If we insist that global touches everything, the concept loses explanatory power.” (Frank, 10, 1-4.)

Martin gives insights into group meetings in and out of the workshops and describes his interactions with his colleagues in this way:

"So from the workshops- We had full faculty meetings where we discussed the importance of the global learning initiative, what needed to be included in all of our curriculum. And so it wasn't as though I talked to one or two individuals. We actually talked to the faculty group. I can't remember anyone objecting to it. Everybody thought it was very positive. And that it was something that should be included. Now I did bring specific faculty members to further discussions because they were going to teach the courses that included the additional international case studies. I am thinking now. .. [Lists various faculty members in different disciplines]...Yeah we spoke about that. There was always a positive attitude towards including them, including it and creating ways to make the global learning a way for pushing for our students. Something positive.” (Martin, 8/7-21) In a final vignette, a respondent implies that where a university faculty member stands on the global learning process is a litmus test as to where one is in meeting their duties. Respondent 4 makes this statement: "Most responsive Faculty are re-inventing themselves, in numerous areas/processes, in order to stay relevant/effective in their 
GLOBALIZED teaching/learning efforts----at least in my own personal case!!” (Respondent: 4, 3/11-12)

Another collective opportunity that university faculty members experience in the global learning professional development workshop is to transform themselves and others by developing multidisciplinary communities through the workshops. Multidisciplinary communities occur when the respondents experience the global learning professional development workshops as an opportunity to make relationships across disciplines in the workshop with the hope of improving research and practice. Examples of the sub-theme of multidisciplinary communities include this statement by Kelly:

"Again it is primarily the perspective that people brought into the workshop. Like some people were from education, some people were there from chemistry. And as I mentioned before my subject [titled omitted by researcher] is very interdisciplinary. It is everything- It is science, it is economics, political science and all that. And that is what it helped me [to do], To develop my course, To come up with the assessments and the course content which is aligned with the global learning course outcomes and meeting the objectives of perspective and awareness and engagement.” (Kelly, 2/1-2/7)

Respondent 26 admits "I developed collaborative relationships with colleagues across disciplines” (Respondent: 26. 3/9).

Multidisciplinary communities can work in many different ways. In describing working with another faculty member from a different discipline in the classroom, respondent 16 said, "We share student videos of their class presentations, receive feedback. We have had Ms. [name withheld by researcher] from [subject withheld by 
researcher] department present to our students on persuasiveness” (Respondent: 16, 3/14).

Another respondent describes how working in multidisciplinary communities leads to breakthrough in their thought processes in this way "The level of interdisciplinary reach of my topic... This epiphany occurred during the workshop when all of us, the participants were discussing various aspects of global learning” (Respondent: 18, 2/33-34).

Another collective opportunity university faculty members have is to channel positive emotional energy as a group into a renewed sense of enthusiasm. The sub-theme of enthusiasm occurs when the respondents experience global learning with a wave of emotional support from others that supports and goes beyond professional norms and seems to affect the emotional state of the respondents and their work and engagement. Examples of the sub-theme of enthusiasm can be found in the statements made by Dan and Melissa:

"Wow. The most engaging part were my fellow faculty members. The people you figure the people that were doing this and maybe because this was the first... These are the really early adapters. The real pioneers. People were really passionate about what they were doing. That is infectious. Anytime you run into someone who is really engaged really excited about what they are doing really passionate about what they are doing and it almost doesn't matter what it is they were doing. It draws you in. So working with the different faculty members in the workshop I thought it was just a blast. It was really interesting to see people that 
were that involved and that passionate about what they were doing.” (Dan, 2/2432)

"Everybody was very enthusiastic- that was probably what made it engaging. That everybody was very enthusiastic. The people in global learning really believe in global learning. They really like what they do. And so their enthusiasm is very infectious. Every time I meet with [Global Learning Initiative staff member], well really anybody there, they are all very excited about anything to do with global learning. So that makes anything you do with them engaging and interesting because they are infectious. You know? [Laughing] God I sound like a commercial!” (Melissa, 2/39-45)

\section{Individual challenges.}

A third way that transformational learning for university faculty members who have attended global learning professional development workshops can occur is in the form of one or more individual challenges. By definition, the theme of individual challenges occurs when the respondent views their experience of global learning as an individual or personal call to do something that is not easy or that goes beyond what they are used to. The respondent feels an internal or external sense to change themselves. Indicators come in several forms. The actual word challenge may be used. Synonyms to the word challenge may be used. The respondent may discuss the difficulty of a task. Qualifications to this theme include several factors. The task or challenge must be related in some way to the goals of the global learning professional development workshops. Although the respondent may mention other people, groups or institutions in some way, the challenges are still centered on the individual. The challenge must be meaningful and 
important. Exclusions to this theme also involve several factors. Challenges that are not related to the goals of the global learning professional development workshops are excluded. For example, statements by a respondent discussing the challenge of facing sickness during the workshops are excluded. Also excluded are challenges directly related to the global learning professional development workshops that are trivial or technical such as the actual formatting of a syllabus.

Examples of the theme of individual challenges can manifest themselves in several ways for university faculty members. Individual challenges can include these statements by Frank and Kelly:

"And I suppose that the challenge, the intellectual challenge is that they had for their own very good reasons a conception of what global meant. And at that time, the courses that I brought there as potential candidates to be global learning courses were not naturally set up to incorporate that sort of thing.” (Frank, 1/21$24)$

"I don't have a direct answer to that. It is not a dilemma. Yeah to some extant especially when we have to convey certain very abstract (ideas). Like even one of the objectives of the global learning is something which is the willingness to engage the students, or a sense of willingness to develop to engage into the global citizenship. It is a very abstract idea. Sometimes still today I wonder how can I make students, to bring to that level because in a class you have students from all different disciplines and from all different levels so how to bring them at par that is a dilemma I still face but the workshop provided me with opportunities to be developed in my course which may have help or is helping bringing them together 
particularly at bringing them to the level of global engagement in that class, how to bring them at par and moving forward particularly at bringing them at the role of engagement.” (Kelly, 2/16-26)

For many of the survey respondents, individual challenges centered on maintaining their standing in a shifting world. Respondent 4 explains that the challenge has to be met, "in order to stay relevant, futuristic, and competitive, and to become effective leaders, in a GLOBAL sense” (Respondent: 4, 1/33).

After reflecting on the notion of bias, respondent 22 typed, "It is a challenge to realize that we have so many prejudices in our own perspective of the world, and others, and particularly towards our students and Miami culture” (Respondent: 22, 2/ 28-29). Speaking of his/her work with students, respondent 11 typed, "I don't remember all the specifics anymore, but I do remember being challenged to reconsider the way that I was engaging my students” (Respondent: 11, 1/18-19).

\section{Collective challenges.}

A fourth way that transformational learning for university faculty members who have attended global learning professional development workshops can occur is in the form of one or more collective challenges. By definition, the theme of collective challenges occurs when the respondents view their experience of global learning as a group or collective call to do something that is not easy or that goes beyond what they are used to. The theme of collective challenge occurs when the respondent feels an internal or external sense to change their social world. Indicators appear in many ways. The actual word challenge may be used frequently. Synonyms to the word challenge may be used. The respondent may discuss the difficulty of a collective task. Qualifications to this 
theme include several factors. The task or challenge must be related in some way to the goal of the global learning professional development workshops. Although the respondent may mention personal or individual concerns in some way, the challenges are still centered on the group or the collective. The challenge must be meaningful and important. Exclusions to this theme limit this theme in various ways. Collective challenges that are not related to the goal of the global learning professional development workshops are excluded. For example, statements by a respondent discussing the challenge of group scheduling conflicts during the workshops are excluded. Also excluded are challenges directly related to the global learning professional development workshops that are trivial or technical such as the actual seating arrangements of the group.

Examples of the theme of collective challenges manifested themselves in various ways. For many participants responses came under the sub-theme of global. The subtheme of global occurs when the respondent sees as or connects the global learning professional development workshops to part of the larger process of globalization.

Examples of the sub-theme of global include this statement from respondent 4:

In a very fast-paced changing world, due to fast-paced technological advancements and globalization in all aspects of life and business/commerce, it was deemed essential and very critical/important, to adapt the way we all teach and learn, pursuant to staying ahead of the "curve" in education and business/commerce----in order to stay relevant, competitive, and successful, in sustainable/effective ways. Given these rationalizations, these GLI workshops provided huge insights to TRANSFORM/CHANGE/ADAPT/RE-INVENT---in 
real-time, all of our pedagogy, resources, and teaching/learning

STRATEGIES/OUTCOMES, in all areas/regimes of EDUCATION.

(Respondent: 4, 1/18-23)

Respondent 22 warns, "We are altogether in a changing world, we better prepare for it” (Respondent: 22, 3/19). Respondent 4 types, "Students need to be consciously and critically looking far into the FUTURE, and deeper into GLOBALIZATION, in order to be predictive, responsive and timely in effectively navigating and adjusting to the fastpaced changes/challenges/opportunities, in all aspects of our CHANGING GLOBAL environments and circumstances” (Respondent: 4, 2/3-5).

A call to leadership is another way university faculty members take on a collective challenge. This sub-theme occurs when the respondent experiences the global learning professional development workshop as part of a call to leadership in and beyond the classroom. Examples of the sub-theme of a call to leadership include this statement by Dan:

"I was a kind of proselytizer among the [faculty member's] faculty for a lot of what I got out of the workshop. I think that is primarily where I have had the most interaction with instruction is with my fellow [colleagues].” (Dan, 7/32-34)

Dan also said the following when discussing another global learning influenced project that happened because of his leadership, "I lobbied for it. I proposed it. I got shot down the first time but we kept pushing it” (Dan, 7/30-31). The specific nature of the project is being withheld by the researcher in order to help maintain Dan's anonymity.

Describing his or her call to leadership and the collective work it entails, respondent 4 made the following statement: 
Due to my personal "ambitious" outlook, and my clear understanding of the impacts of GLOBALIZATION, and my profound desire to be a "changeagent" and Leader in this cause, I was/became more confident in the process/effort of re-inventing myself---as a Faculty/Mentor/Leader in our community, and as an effective ADVOCATE for Global Learning Initiatives!! (Respondent: 4, 3/34-37)

\section{Research Question Three}

Research question three asked: How do university faculty members who have attended a global learning professional development workshop and report having had a resulting transformational learning experience apply this transformation to their teaching?

\section{Qualitative findings for Research Question Three}

University faculty members apply their transformation to their teaching in four ways. These FIU faculty members applied their transformation to their teaching by first exploring the theme of conceptual change and the related sub-themes of student centrism, content and knowledge. The second way that FIU faculty members applied their transformation was through individual instructional change modeling learning resources and authentic global learning. The third way that FIU faculty members applied their transformation was by developing systematic awareness and the related subthemes of fulfilling the promise and support systems. The fourth way that FIU faculty members applied their transformation was through the theme of systematic change through instruction and the related sub-themes of bridging diversity and moving beyond the global learning classroom. 
The following chart identifies the major themes and sub-themes as they relate to research question three.

Table 10

Major themes and sub-themes in response to research question three.

Themes

Conceptual change

Individual instructional change

Systematic awareness through instructional change

Systematic change through instructional change

\section{Sub-themes}

Importance of teaching

Goal of teaching

Student centrism

Content and knowledge

Modeling learning resources

Authentic global learning

Fulfilling the promise

Support systems

Bridging diversity

Beyond the global learning classroom

The following passages describe the major themes and sub-themes in the findings as they relate to research question three.

\section{Conceptual change}

The first way university faculty members apply their transformation to their teaching is by exploring conceptual changes in the way they see their instruction. By definition, the theme of conceptual change occurs when the respondent changes the concepts that drive instruction which in turn change the nature of their instruction. In 
terms of indicators of this theme, the language used in the data may mention terms such as thought, idea or synonymous terms. Qualifications to this theme include several factors. While the concepts may directly mirror the work of the global learning initiative, these concepts are fully realized and adopted by university faculty members after confronting the individual confronts them and they perceive the collective opportunities and challenge. Concepts are made personal and owned by university faculty members after they have been filtered through critical self-reflection and social discourse to fit their individual and collective needs. Exclusions help set clear limits to this theme. Statements that do not develop from the critical reflection of university faculty members are excluded.

Examples of the theme of conceptual change developed in many ways for university faculty members including exploring the importance of teaching at the university. The importance of teaching as a sub-theme occurred when the respondent felt that the global learning process has led to a renewed focus on the importance of teaching at the university. Examples of the sub-theme of the importance of teaching include this statement by Frank that illustrates why making teaching important is essential:

"I think that overall that the global learning experience has been beneficial because it has forced me to consider how I teach and I think at the end of the day, that all of us probably have to do that even though FIU doesn't really care about teaching. I think it is incumbent upon professors to give serious thought on that and to figure out how they can do that part of their job better.” (Frank, 6/23-27) Respondent 32 explains how the workshops are different to the university culture as it deals with teaching, "Teaching at FIU is usually a more or less solitary venture with 
no one looking over your shoulder. This is the first time I had to submit my ideas to anyone but my students” (Respondent: 32, 2/18-19). The importance of teaching cannot just be tied to meeting performance goals but also should be related to the idea of better understanding student learning too. Kelly explains this notion in the statement:

"That students comments about my courses is a testimony to my course, my global learning course. The workshop has certainly helped. I have gotten really good comments from my students. Some of the things like from the beginning of the course, I do ask them what they know about these global (discipline withheld by researcher) topics. At the end of the course I take another survey and I can ask them what do they know now after going through this global learning course. The comments are very gratifying and it is very rewarding to listen to the students. It is rewarding to read their comments of what they have learned and what they have learned from this course and what kind of perspective they have got through the global learning course.” (Kelly, 4/34-42)

Frank describes the critical reflection on this concept that he went through.

"I suppose it would be that I had thought of myself as a fairly competent teacher. That I got good teaching evaluations. And I think that I concluded that it was more the energy and perhaps the personality that I had put into courses rather than an effective teaching method that I was using. I had to reconsider what it meant to be a good teacher. Was that an amusing teacher? Was that a teacher that keeps you awake? Or was that a teacher that got you deeply engaged in the material?” (Frank, 4/12-17)

University faculty members make it clear that concepts influence the choice of 
education methods and instructions. "I walked out of the workshop with various specific pieces of valuable and practical information and tools. However, the most important reflection was a remarkably simple thought of profound implications, the idea that learning is the goal of teaching." (Respondent: 24, 1/ 27-29)

Respondents made statements that show how critical teaching is to student learning as in: "Made me think about the ways in which teaching contributes not only to knowledge per se, but also ethics” (Respondent: 26, 1/17). In writing about the workshops, a respondent implies how for some faculty members the importance of teaching not only enriches the student but the faculty member too.

It brought awareness about the importance I have as an instructor and mentor...It brought awareness about different methods and techniques on how to improve my teaching...It brought awareness about the importance to prepare our students to a global world. It enriched my own life by educating me on the importance of not only being international but actually (a) global citizen. (Respondent: 22, 1/ 17-20) The renewed focus on teaching is not just an individual act but one that comes from interacting with others at the workshop. This is true even when the experience is negative. As a respondent wrote:

Again, I (along with the others in the workshop) felt that one of the requirements of Global Learning contradicted our ethical standards....It made me realize how little teaching is understood in higher education and how little sense of specific mission individual professors have... I realized that I was better at teaching than I thought and that I have strong principles in my work. (Respondent: 6, 2/28-29, 2/39-40, 3/27) 
Parallel to the importance of teaching for university faculty members is the goal of teaching and the steps that lead to it. This sub-theme of the goal of teaching is a conceptual break from focusing on what content you teach to instead also asking how to teach content, why they are teaching content, and towards what outcome. Examples of the sub-theme of the goal of teaching include this insight from Dan:

"The other thing too I thought that was useful too was the notion of sort of reverse engineering your outcomes. Look at what it is you are trying to accomplish through an outcome and to make sure what you are doing leads to that goal. That has been very valuable too.” (Dan, 4/45-5/2)

Melissa's discussion implies that the focus in the past was too much on the content and that the outside influence of the global learning process has disrupted this pattern:

"I think possibly it just made me think about in more complexity about how students were going to learn because I had never done anything in the form of global learning. So I never had, I mean outside of global learning, you are just given a course to teach. There is no expectations set up for you externally really. We have that template thing at the end of the course where you talk about each of the three objectives and how you met them and what you think happened. I forget what the form is called but you know what I am talking about. Something like that makes you think about what you are doing in a class and why you are doing it. And so I had to think a lot more about why I give certain assignments and what the purpose is and what do they accomplish.” (Melissa, 4/33-41)

Frank shows that individual faculty members are in different places when it 
comes to the goals of teaching. For Frank the issue seems to be more on how to teach his content when he says, "One of the things that was helpful was their emphasis on active learning methods. They insist on it in fact. So it requires you to think hard about how you teach and not just what you teach” (Frank, 2/22-25). In discussing how to meet global learning goals, Kelly implies that there is a difficulty if an individual faculty member is trying to make this happen alone and consequently, this reveals the need for university faculty members to collaborate collectively to meet the goals of teaching. She says, "Sharing the ideas and specifically I would say that global learning has three aspects which is the goal to impart a global perspective, global awareness, and global engagement. And people had different ideas on how to engage the students and that discussion was very helpful in developing my global learning course.” (Kelly, 1/22-25)

Faculty members often have several factors to consider when deciding what to teach and how and why as necessary steps in the goal of teaching as the following statement suggests:

As stated in the answer to a prior question, though I knew this to be true, the workshop cemented the idea that learning is indeed the goal of teaching. Therefore, as an Instructor developing a teaching style that is best suited to both the course objectives and students' needs, and learn and apply what research tells us about effective teaching are crucial aspects of supporting an effective process. (Respondent: 24, 2/34-37)

Respondent: 19 concurs, “I think more comprehensively -- and globally -- about the teaching methods and content of my courses, including those that are not designated 
as Global Learning. The instruction is more interactive and the content takes into consideration more of a global view” (Respondent: 19, 1/17-19).

The other sub-theme in the analysis of the data under the theme of conceptual change is the notion of student centrism. FIU uses a learner centered approach in the global learning professional development workshops (Green, 2013). The broader term student centrism is used for this sub-theme to not only encompass a learner centered approach but also to describe the implications of a focus on students translated into the contexts, applications, and meaning schemes of faculty members and the influence this has on individuals, groups, ecologies, and systems. Student centrism is briefly introduced here but the larger implications and influence of this concept is indirectly alluded to and informs the discussion of the other themes and sub-themes to be mentioned later in the findings. Examples of the sub-theme of student centrism include this statement by respondent: 21 who explained the conceptual change this way:

I have realized that the center of focus of the class is not the professor but the students. It is not important that the professor is knowledgeable and gives a flawless lecture -- it should emphasize student activity, participation and thinking. (Respondent: 21, 2/33-36)

Respondent: 27 wrote of the conceptual change "I think it caused me to increase the role of student engagement in my classes” (Respondent: 27, 2/15). This statement by Kelly shows how this conceptual change manifests in practice:

"I did, the workshop helped me in that it is okay- most of the courses are what the students take, how you can goad them, or basically how you can bring the student to make them, or get them ready to do something. That is the global engagement 
part. So the global learning workshop kind of helped me to highlight the importance of engagement. It is not just imparting the global awareness and the global perspective. How the students can be engaged into making a difference in society and going forward just willing to engage into the community, the university, or in their own community. This is the one thing that I got out of this global learning workshop.” (Kelly, 3/25-35)

Respondent: 16 translated the conceptual change into this practice in his or her specific classroom in a statement.

Recognized the need for students to understand Global Success. Also recognized the need to motivate them to have a persuasive speech...We bring the concepts of global success, the students present an approach to achieve it, we provide feedback, students update their work. (Respondent: 16, 1/18, 3/1)

For many FIU faculty members this conceptual change meant looking at students in a different way. Respondent 14 expressed the concept of student centrism as "the reinforcement of students as resources” (Respondent: 14, 3/3). A respondent who has worked at FIU and has helped development in Latin America for several decades said this of his/her background:

This gives me a certain perspective and substance regarding international/global issues. However, teaching this content presents other challenges. Making global development relevant to the students today is important. Many of our students come to us with a variety of international backgrounds, directly or indirectly. It is important to recognize this as a strength, as a tremendous resource. In the workshop I attended I came away with appreciating several new techniques that 
will enhance my teaching in the area of global learning. Only one of which, by example, is modifying my syllabus and outside assignment to capitalize more on the strength and resources presented in my students. (Respondent: 14, 1/27-35) The following statements show that other university faculty members individually and collectively see the value of student centrism.

Since I teach many courses and personally I value students' participation in class discussion etc. I believe that all input is important which advances the discussion further and enriches global learning perspective. (Respondent: 18, 1/36-37) Writing about the social discourse amongst various faculty members, respondent: 18 continued:

Indeed, all of us shared our experience in working with a diverse pool of students in different disciplines at FIU. And that experiences helps to enrich your course offerings and incorporate new ideas to improve learning environment for students. (Respondent: 18, 3/5-6)

Student centrism for many faculty members means that students are not subjects or objects but human beings who take what they learn and apply it outside of the classroom. For example "It helped me think about my students as actors in the world" (Respondent: 26, 1/35) and

I actually found that GL event where the international students spoke to be very informative. Learning about the different expectations for students' learning, behavior, ideas about plagiarism, etc. were very helpful ideas to ponder. I think that has opened up my mind quite a bit. I would like more similar presentations on 
these topics. Understanding why my students might see things differently is very helpful. (Respondent: 27, 2/2-5)

Respondent: 14 summarized how the concept can influence faculty members collectively in the statement "I think we all came to realize that we need to re-evaluate how we teach to the waves of students who come from such varied backgrounds and can enhance our attempts to have student increase their critical thinking.” (Respondent: 14, 3/13-14)

Many respondents recognized how much this conceptual change influenced learning and began changing their practices. "I try to form a partnership with the students" (Respondent: 13, 1/35) and the "more participation and collaboration with the students in class....the more students are directly involved the more they learn” (Respondent: 33, 1/37, 2/8). Another respondent wrote: "Specifically, in the realization that planning the sort of activities that enable students to engage with the learning by having some fun and developing a sense of belonging, facilitates the success of the teaching/learning process” (Respondent: 24, 1/ 38-39). Respondent: 10 stated “I believe the additional assignments caused some transformation for some students” (Respondent: $10,3 / 12-13)$.

Student centrism also led to many university faculty members mentioning a move beyond lectures when moving away from passive instruction:

"I guess, not guess, I was just much more old school. I would lecture and sometimes group work but group work in the worst way groups could work together where one person is doing all of the work and everyone else coasts along and you never really resolve that unless you really engage the teams properly but 
anyway a lot of what I was seeing in this workshop really caused me to rethink my approach to instruction And to really think about instruction in the context of all the technological changes that have been going on.”(Dan, 2/43-3/3)

Respondent 21 reflects, "My teaching style: hour long lectures with power-points and four exams -- is not really imparting proper education to the students” (Respondent: 21, 2/21-22). Respondent 22 details the change in instruction with this statement:

I had to be open to students perceptions, cultures, dreams and goals. I modify my "lecturer" position towards a dialogue, where I have to know, understand my students, and not only preach my knowledge.... Has been a great transformation of my teaching and my person. Being aware of the others has resulted in a personal growth. (Respondent: 22, 2/ 4-6)

Respondent 21 reveals an epiphany in another statement "Kind of...the realization my lecture is nothing -- it is the student learning that counts :-( " (Respondent: 21, 7, 3-5). University faculty members in this study often discuss a change in how their content and knowledge is viewed and imparted as another important aspect of conceptual change. This sub-theme of content and knowledge occurs when the university faculty members changes the way they view, teach or apply their subject matter or knowledge due to the global learning professional development workshops. Examples of the subtheme of content and knowledge include this statement from Respondent: 15 who wrote, "I became more aware of my subject from a global perspective. I never thought about my subject globally or how faculty in other countries teach that subject” (Respondent: 15, 1/17-18). Dan explains the transformation of content and knowledge as part of the problematization and transformation of the global learning process: 
“And we haven't been in the [faculty member's] profession as good at coming up with the idea of what is it that we want students to do with all this knowledge. We are sort of a process oriented profession...But this is again a more theoretical or conceptual approach to what it is you want students to know. You want students to know what scholarly communication is all about. What scholars do and what they as students and potentially on up the academic food chain, what their part and what their role in that scholarly communication is. In that environment, in that world." (Dan, 5/ 4-28)

Several other university faculty members reflected on their content knowledge and how their transformation related to conceptual change. Respondent: 7 wrote, "It caused me to think more broadly in my content” (Respondent: 7, 1/29). Respondent: 29 wrote, "I have incorporated content that interlaces domestic views with international views" (Respondent: 29, 1/29-30). Respondent: 20 wrote of his course, "The content of the course is dynamic; therefore, with current events and learned techniques I have improved the delivery of the course. There is more interaction among students than previously (flipping the classroom techniques)” (Respondent: 20, 1/29-30). Respondent: 22 "explains that:

I always used international examples in my class, but never got to the level of engaging in the discussion of awareness, perception and engagement with students. The reading about the meaning of global perspective was very important. Now my exercises are not only descriptive of different cases around the globe but analyzing their similarities, differences and how each student perceives those differences and similarities, do they identify with different 
solutions? Do they reject or accept different cultural responses to similar

problems? Do they care about the topic in class? (Respondent: 22, 1/ 30-35)

Respondent: 20 states that "I feel that I am now presenting the subject matter according to the latest teaching methods” (Respondent: 20, 3/35). Respondent: 11 wrote, "I do remember thinking differently about how I approach the content and changing some of the things that I cover" (Respondent: 11, 1/ 4-5). Respondent: 27 explained "Most of my courses tend to be Global in scope, but it has helped me to be more meaningful in the contemporary applied analysis of topics” (Respondent: 27, 1/ 31-32).

\section{Individual instructional change}

The second way that university faculty members apply their transformation to their teaching is through a change in their individual instruction. By definition, the theme of individual instructional change occurs when the respondent changes the act and/or mode of instruction. Indicators manifest themselves in several ways. Synonyms for change are used often used in relation to instructional change. The respondent often cites the way they used to teach in comparison or contrast to the way they presently teach. Qualifications to this theme limit this theme in important ways. These are changes that come from critical reflection and social discourse that occurred in the global learning professional development workshops. Exclusions to this theme are also important. Although the conception of teaching may inform the act of teaching, statements in this theme deal more with the application of teaching. Statements were excluded if they did not focus on change centered on the individual university professor and their instruction.

Examples of the theme of individual instructional change can appear in many forms. University faculty members model tools and strategies as part of how these 
university faculty members apply their transformation towards their classrooms The subtheme of modeling learning resources occurs when the university faculty members see the global learning professional development workshops as an event inspiring new forms of instructional methods and strategies as learning resources they can use to apply their transformation towards instruction. Examples of the theme of learning resources include this statement by Dan who was able to use social discourse with faculty members combined with insights from other sources to fit his needs:

"Wow. There were a number of different speakers that came in particular the gentlemen who did the work on team teaching. We had an all day session on that which was excellent. So I thought that umm the bringing in the experts like that It could not have been cheap. But the man you know was very good at what he did. And So the different perspectives from a lot of different speakers, a lot of different types of instruction, I thought it was very useful and it was like us talking within each other which a part of it was useful but it was also bringing in the different outside experts to really bring a focus on some of the aspects in developing the syllabi.”(Dan, 2/10-17)

Melissa was able to use technology at the workshop to model the work of other faculty members and make it fit her own needs. Melissa explains:

"Well one thing I specifically remember we went over on the website, the different parts of the website, in the section for faculty, which had other faculty's' planning and syllabi and things I could then use as a resource to help create my course and to create things to meet the three global learning objectives. I remember I was going over that and thinking that that was going to be so helpful I 
was so glad they were showing me that because here I was trying to understand those objectives and meet them, that these other people had done it now that was so helpful. I could look over their ideas and I could get ideas and I did get ideas.” (Melissa, 2/22-29)

“The workshops gave Dan a second look at another resource:

The other thing is that the workshop did a lot of emphasis on rubrics...I was always adverse to a rubric approach and I still think, they are not the magic bullets. They have more of a role in than I gave them credit for before the workshop. So now we use rubrics in most of the writing assignment in the (global learning) classes. I think they are very effective. “(Dan, 7/3-9)

Martin explains how he took a resource from the workshop and began to use it with other faculty members outside the workshop:

“...in those workshops, we did go in to discuss the pedagogically, the pedagogic elements of how should we teach these courses. And one of the things that came up was that we should include international case studies into the curriculum, into current courses that could be adapted to be part of the global learning curriculum. So that was good stuff that came out. When I came back after the workshops, we started putting into some of the courses that we thought would be a best fit, international case studies that could be then included as part of the global learning curriculum. “(Martin, 4/38-45)

Martin’s statement implies the fact that learning resources had to be made to fit the circumstances of each faculty member's needs. Faculty members did not just automatically adopt all that was presented at the workshops. Faculty members often 
picked and chose what resources they could use from the workshops. Faculty members also made decisions about to what degree they would use these resources, when they would these resources, and how they would use these resources. Respondent: 13 wrote that the workshops inspired "Inclusion of activities that I would not have considered...Stimulating discussion at the workshops provided some ideas for future use...I was intrigued by group learning” (Respondent: 13, 1/18, 2/7). Respondent: 21 wrote "It is an ongoing and slow process -- but I am moving more towards active learning -- less on big midterm and final exams. I am actively thinking of offering flipped courses ...” (Respondent: 21, 3/10-11). Respondent: 27 explains “I really like the Visual Thinking Strategies that they use, and I use it in my classes now. I also found the GL workshops to be very validating, in that they support varied, student-centered pedagogical strategies” (Respondent: 27, 1/ 20-21).

Some faculty members adopted the learning resources given to them in formal ways as a way of updating instruction. Respondent: 20 wrote that the workshops "Gave additional modern techniques in presenting the material to the class and preparing examinations” (Respondent: 20,1/19). Many other faculty members adopt or model learning resources in informal or unique ways. Sometimes faculty members seem to adopt facets of the workshop themselves such as the use of guest speakers. Respondent: 2 for example noted of the global learning course he or she teaches "I seek out guest speakers and plan out of classroom activities which facilitate learning outside the classroom" (Respondent: 2, 3/7). Kelly has been modeling his own global learning course as a resource for developing his and other faculty members' other courses. Kelly says "And in fact this that, this course, the global learning course has been a resource for my 
Masters and PhD level courses” (Kelly, 4/16-17). Respondent: 20 shows the modeling of tools and strategies from more than one discipline into a global learning course:

I emphasize Bloom's Taxonomy in addition to the objectives of the Landon School of Business. Every student starts with developing the knowledge and comprehension of the course material, as well as completing research assignments which require analysis, evaluation and synthesized delivery to their classmates. (Respondent: 20, 2/16-19)

Another important part of modeling tools and strategies for instructional use for the university faculty members is the insistence of the use of authentic global learning. Authentic global learning are instances where the respondent feels that global learning has helped create opportunities in instruction for students to do something that is applicable to what they will do in the field or real world. Examples of the sub-theme of authentic global learning includes the following statement excerpt where Melissa discusses moving from a traditional paper to much more hands on relevant assignments incorporating different international resources and perspectives leading to work that is closer to what students would expect in the field.

"I think they learned more that way than doing a standard research paper. Frankly thinking about it, once you graduate college, how often do you write a research paper? Pretty much never unless you are going to be a college professor.” (Melissa, 8/16-18)

Dan discusses how the instruction developed at the workshops mirrors developments the major institutions in his field have currently begun to promote: 
"So that is something that is interesting because the workshop tipped me off to that approach that sort of reverse engineering approach before the [faculty member's] profession had really caught up. So now when I am teaching a class my main thought is not what I teach them but what do I want them to be able to do when they walk out the door. What do I want them to be able to do? What is the outcome? And then what I do in terms of instruction and activity is basically designed to make that outcome happen. So I think that is an approach, that kind of reverse engineering approach, that has been very useful and I definitely got that from the workshop. (Dan, 5/30-37)

This excerpt from Martin’s interview describes how much student work can mirror real life applications:

“And those team projects there were typically based on case studies and those case studies were mostly United States based case studies and we changed that so that just in about every senior level course, the case studies were moved to become international case studies.... Case in point: The product Tide...In Venezuela and in Cuba too. But in Venezuela, it was ACE or ACE The same exact product for whatever many years ago it was called ACE . ACE. And Ace was a product that -so based on the research the students came up with- one of the student teams. Umm. They found that in Venezuelan communities it was better instead of putting Tide in Miami like in Weston and Doral and all that to actually put in Ace on the shelf. So, and interestingly enough, so that was based on a student project. A year or two ago I heard from, well my brother works for Publix, and I never told him about this project, but I heard from another friend and then 
my brother confirmed, he works for the corporate headquarters of Publix that Publix in Weston started putting Ace on the shelf and they were disappearing. There were putting twenty boxes of Ace next to Tide and the Ace was gone. So the insights that the students came up with that project by projecting the US Hispanic market here and doing studies, doing research internationally into Latin America, they came up with that as one of their strategies. They combined international with U.S.” (Martin, 5/28-6/25)

Implied in a statement by respondent 21 is that students need to be ready to work individually and collectively:

Most importantly, it reminds me all the time is whether the students are learning to use the knowledge imparted in the course for decision making and problem solving in the world around them...The main change has been an emphasis on group projects focused on real-life problems of the world. (Respondent: 21, 1/ 17$18,1 / 28)$

A statement by respondent 2 implies that university instruction was too focused on theory: "I now focus on both theory and real world applications...Students don't just need theories. They need things they can relate to.” (Respondent: 2, 2/3, 6/17)

A statement by another respondent makes it clear that combining theory with real world applications makes instruction that is more engaging for students. "It caused me to create experiences that are deeper and more meaningful.” (Respondent: 7, 1/19)

\section{Systematic awareness through instructional change}

The third way that university faculty members apply their transformation to their teaching is through systematic awareness. By definition, the theme of systematic 
awareness occurs when the respondent tries to develop and apply instruction in a way that leads to greater awareness, understanding, interactions, or recognition of relationships, networks, interconnections, elements, hierarchies and/or systems related to the university system and/or Florida International University in particular. The respondents may use the word university or system as indicators of this theme. Qualifications to this theme appear in many ways. The systems of the university may be formal or informal but a qualitative description of them has to be illustrated or implied in relation to the pursuit of the application of instructional change. Exclusions to this theme include statements that merely describe the hierarchy or organization of the university in technical terms.

Examples of the theme of systematic awareness through instructional change for many university faculty members often focuses on fulfilling the promise of the university. The sub-theme of fulfilling the promise is based on the notion that the university has a spoken and unspoken promise to prepare students for the future in terms of global learning. For many university faculty members, this sub-theme is unique to Florida International University. Examples of the sub-theme of fulfilling the promise include this statement by Martin on global learning:

“I think it has been very good for our students. Before we didn’t have that strongwe have always been Florida International University. (However) If you go back five years ago, there really wasn't a focus on international in our curriculum, we had it in the name but in our courses there was very little international focus. Well now there is a lot more elements of that. So I think it has been very good.” (Martin, 7/15-21)

What is perceived by university faculty members as part of the promise of the 
university and what is perceived as part of global learning may be debatable in different contexts but these perceptions are a reality for many university faculty members. Another statement by Martin where he offers constructive feedback illustrates this notion of perception is reality while illustrating FIU's place in the larger system of North American universities:

"But you know, it is one thing to offer, within the curriculum, some courses. I think we need to do much more to offer international experiences. I think last semester- or last year- we only had 890 students study abroad out of 54,000. And we should have three- four- five- ten thousand students that every year go out... Let us just say that $5 \%$ of the 50,000 - the 54,000 should be involved in an international study abroad program. Five percent of 50,000? That is 2,500 students and I would argued that that number should be even higher. You have universities like Georgetown and others that specialize in international diplomacy that $30 \%$ of those students are doing something at the international level. And we are here at less than a thousand and we are Florida International University. So my feeling is that we as a university need to do more to push international programs.” (Martin, 8/30-9/11)

Respondent 24 understands systematic awareness at the university as going hand in hand with instructional change. The university faculty member wrote "I understood better my role as a carrier of the vision and mission of the University Global Learning” (Respondent: 24, 3/17).

Working with and improving support systems within the university are issues brought up in the responses of many university faculty members. The sub-theme of 
support systems occurs when the respondent that the university through its global learning initiative staff or other resources has created some elements in the system that is useful to the success of promoting the concept of global learning and strengthening global learning practices. Examples of the sub-theme of support systems can vary. The respondents will often note the elements of a support system like the global learning initiative before explaining how particular resources provided to them through the workshops have affected their thinking and/or practice. Dan explains such a situation in the following statement:

"I took a lot out of the workshop and also the little mini-conferences they hold. Also the little mini-workshops they have, the blasts, and everything. So I have taken a lot of ideas and a lot of different things. In fact, the stuff I am moving around is from one of the sessions where teams got a miniature whiteboard...this allows the groups to put a little bit more of a qualitative answer or maybe be a little more free in expressing themselves on the whiteboard for an answer or for making a point. That is something I directly got from the workshop as an idea. A lot of little things like that and as well as the broader and more conceptual approach to pedagogy. I definitely will give credit to the workshop. Kind of ongoing stuff the global learning initiative is doing since. “(Dan, 6/15-26) Melissa became aware of how the staff of the global learning initiative were invaluable to her work. She said:

"Just that the global learning program is great. The people who run it are so dedicated. I called them up and said I would like some advice. I was really trying to improve the course assignments for two years. I really had to develop 
something. I sat down with (a global learning staff member). I must have been in her office for two hours. Just her and I brainstorming. Whatever you need, they are there.” (Melissa, 9/19-24)

This excerpt from Dan's interview highlights the graders as part of the support system of the university that is depended on by faculty members and which need help: "Also because they are large classes, usually more than a hundred students. We invariably have graders who do the grading. I think the rubrics are helpful for the graders so that they have a better idea of what is happening in these writing assignments. As opposed to saying it is really well written so I will give them more credit. We only give them five points instead of fifteen points or whatever so I think the rubrics are good for our larger classes. Where we have our graders who may not know what you are trying to get from instruction without them. Those were just a couple of things that I definitely carried with me.” (Dan, 7/916)

An excerpt from Martin’s interview shows how global learning creates a support system that links the university but also students to business and professional networks: "I think it has affected it very positively. Very positively. It forced the university and every single academic department to put in courses that would make students think internationally, to make students to think globally, that makes our students, and I see that here in my job at (the university), that makes our students much more attractive to employers, when employers come in and start to interview our students and the students project an understanding of international issues, it makes our students much more attractive to employers.” (Martin, 7/4-10) 
For Kelly, the convenience of the global learning support system allows Kelly to tap into other faculty members as resources.

"Yeah. What I would like to add is that the global learning workshop allowed me to understand the collegiality across the campus which I got aware of the expertise across the campus that can be approached to improve the global learning environment and global learning outcomes and the workshop had been helpful in developing this ecology of experience.” (Kelly, 5/27-31)

The following interview excerpt shows how a support system in the university may clash with a lack of support in another aspect of the university system.

“Although you may hear differently, teaching really doesn’t matter at FIU in any significant sense. I mean people will give lip service to it. But as a practical matter, people are not rewarded for their teaching. They are rewarded for their research and anytime spent teaching is time away from research. So what that means is that a good career strategy is to devote just as little time and energy to teaching as possible. To do the bare minimum. Well in the global learning course, they are very interested in the methods that you are using, you are having to assess students, they encourage you to use rubrics and all these sort of things that really requires time and effort. And something you wouldn't normally do if you wanted to get ahead on your career at FIU. Now I would be happy to be wrong about all of that and if the global learning people want to show me somebody who was promoted on the basis of their teaching, then I will be happy to concede my point but I am pretty sure that is not going to happen.” (Frank, 2/3-14) Global learning discussions can also highlight what is lacking in the support 
system: "The rest of the university, the deans, the department chairs needs to be pushing for more students going abroad or finding some other way to give an equivalent experience. “ (Martin, 10/7-9) Kelly stated how much the individual faculty needs other faculty members to work as a sounding board:

"It has been wonderful and especially, umm, it was an opportunity to talk to the colleagues who were there who also teach different courses or who are planning to develop a different course and so it was good to have a different perspective into global learning or to developing a global learning course. Since my course is very interdisciplinary, of course it is very science heavy. But people from the different backgrounds brought different perspectives and which help me on developing my course onto global learning- on developing a global learning course which is primarily on the global (discipline withheld by the researcher).” (Kelly, 1/9-15)

Martin's statement showed also that groups of faculty members can be just as limited in their thinking as individual faculty members if they do not interact with the larger community of faculty members and global learning experts in the support system: "We went in having a certain perception of the course or courses that we could adapt to be a part of the global learning initiative, Umm, a global learning course at our school. And so the workshop, did sort of, well, not that it inspired but you could say inspired, it made us think beyond and see how we could put in these case studies. And what we could do to make the courses we wanted to be the global learning courses much more focused on global learning. So yeah in a way it did inspire. The workshop did. “(Martin, 5/10-16) 
University faculty members may note how individuals, documents, departments, and other resources are linked in a network across the university made possible by the workshops. Respondent 4 illustrates this when this faculty member asked for "More effective collaboration/cooperation with librarians, library resources, Publishers, online research efforts, and in "tapping" more into FUTURISTIC Journals/Resources---pursuant to better predicting/responding to future GLOBAL trends/issues” (Respondent: 4, 3, 22-24).

Respondent 4 indirectly explains how the global learning courses help link the university to the local public school system when the university faculty member describes the classes he/she teaches: "Each and every term, at FIU and/or as a Dual Enrollment (DE) course, currently offered at M-DCPS High Schools, with ongoing strategic plans to expand our FIU DE/GLI course offerings to other local School Districts' High Schools.” (Respondent: 4, 4/4-6)

The next two statements from respondents highlight how the global learning initiative works and inspires work with other institutions at the university. Respondent 20 wrote:

Global Learning workshops together with other workshops taken through CATThe Center for the Advancement of Teaching at FIU have helped me in presenting the course material in a more dynamic manner, which have helped the student learn and retain the course's objectives. They research and present the material more often in order for them to "learn while they are teaching”. My role is to enhance on the topic being learned with additional knowledge and material, 
including cases, based on my knowledge and experience of practicing and teaching the subject matter for over 30 years. (Respondent: 20, 2/1-6)

Respondent 22 discusses how the workshops led to other initiatives within FIU:

I decided to engage in the CAT workshops, I realized that there is much to learn rather than much to teach. My path, initiated by the Global Learning workshop put me in the CAT educational revolution ending up bringing me to SBER and STEM institute development.(Respondent: 22, 2/16-18)

Several FIU faculty members have started to both become more aware of the systems within the university and use it to network. Respondent 7 wrote, "I have developed relationships with individuals in the GL office and across campus” (Respondent: 7, 3/10). Respondent: 18 noted that global learning brought "awareness about the wonderful support system and a sense of participation in the improvement of global learning experience for students” (Respondent: 18, 3/28-29). Respondent: 29 initial work in course design led to other activities:

Discussions with other colleagues and workshop guidelines were useful in addressing students' needs and most effective class design... Process connected with the urgent need to incorporate global perspective in our classrooms and this fact made me connect with colleagues to advance in more inclusive projects.

(Respondent: 29, 1/ 18-19, 3/2-3)

\section{Systematic change through instruction}

The fourth way that university faculty members might apply their transformation to their teaching is through systematic change. By definition, this theme occurs when the respondent gives responses that suggest or implies that their pursuit or application of 
instructional change is a kind of activism that influences systems that influence the systems related to the university or that go beyond their global learning course or the university itself. In terms of indicators to this theme, the respondent may seek to connect instruction to institutions, relationships, or concepts in the world. Qualifications to this theme can include several elements. The changes sought are for the betterment of students learning, instruction, the community, and the global learning process. An important exclusion to this theme are statements that describe the changing of superficial issues. The changes sought cannot be trivial.

Examples of the theme of systematic instructional change can center on bridging diversity. The sub-theme of bridging diversity are occurrences where the faculty member reflects on their diversity or that of others and feels a need to improve the situation. Faculty members may also feel that their own experiences with diversity affects how they deal with diversity at the university. Examples of bridging diversity include this excerpt from Martin who discusses the importance of activities that bridge diversity in his classrooms:

"And understanding that that cultural background is going to make- these individuals are going to interpret the same issues and the same things they are learning, It is going to be interpreted differently because of that cultural background...So I tell them all keep in mind that when they are talking about an issue you may think they are being perfectly clear but The other person over here may think he or she are being perfectly clear and you have two different understandings of the same things...So we have exercises that shows that. They think they are being perfectly but they have different views of the same thing. 
That is why I think global learning was critical and is important for this university. It may be different for a university in Oklahoma or Nebraska or Minnesota but for this university, Florida International University, it is critical.” (Martin, 14/30-14/41)

In this excerpt Kelly explains the importance of assessments in bridging diversity. "Yes, to some extent. It did help me to think about different assessments. One which I actually implemented that is that I cannot rely on just one assessment because all the students come from a different level, from a different style of learning. And so my intention and my objective after the workshops has always been that if I can provide multiple assessments or different assessment opportunities. Some of the students can do well in the multiple questions, some of the students can do well in the short essay, some of the students can do well in the discussion, some of the students can do well in the presentation. That is the thing which came out of the global learning workshops and I do try to incorporate all different kinds of assessments. So the students, even though most of them will do at least something better in one or the other assessment styles. "(Kelly, 3/43-4/6) Respondent 2 had to take on a new role to begin the process of bridging diversity. The faculty member wrote, "It removed the pressure I had of having to know everything and assuming audiences had certain expectations of me and the content of my teaching. I realized that my background, experiences and skills while being distinct from the typical American, actually enriched their learning” (Respondent: 2, 3/17-19). Respondent 27 noted a diversity blindness amongst faculty that was starting to change: 
I think this program is a huge asset in such an international university. We have students from all over the world, so it seems to me that the GL program is helping the faculty catch up to that and be more conscious of our colleagues and students' diverse backgrounds. This awareness is critical for the faculty so that they can guide the students more effectively in the global workforce. (Respondent: 27, 3/ 16-19).

The global learning process led many to reflect and act beyond their renewed focus on diversity. Respondent 10 wrote that the global learning process led to "Increased use of curriculum and strategies directly related to cultural diversity/sensitivity...In reality I already knew how limited my social circle is. However the global learning workshop really drove it home" (Respondent: 10, 1/17, 2/18-19). Respondent: 22 adds, "The world is not flat, and we rather open our mind to the "other" and the change if we want to really make a difference as a professor, mentor, or instructor, and for the matter, if we want to live a real fulfilling life” (Respondent: 22, 3/7-8). Respondent: 24 suggests that "Sharing ideas and different points of view is always a productive experience" (Respondent: 24, 3/7). Respondent: 10 wrote:

I became more sensitive, but also more curious and less reluctant to query students about their cultures...I need to find ways to grow my social networks to encompass people from diverse cultures...I believed I functioned in a more diverse network; in reality, my social network is limited to friends/family who are (culturally) like me. (Respondent: 10, 1/37, 3/1, 2/ 30-31)

Respondent: 14 explains the collective dilemma as: 
We all approach learning with certain perspectives based upon our own experiences. These experiences can be enhanced by incorporating the experiences and perspectives of others. As instructors we need to ensure that the structure is available for this dialogue. (Respondent: 14, 2/13-15)

Bridging diversity happens in many forms. Kelly bridges the diversity that is between graduate students and lower division students in an interesting way: "I have found my class is especially fascinating. Because I also teach the Master and $\mathrm{PhD}$ level courses. And in fact this that, this course, the global learning course has been a resource for my higher level courses. I have the engagement part which I took from the global learning workshop and I am incorporating into my higher level courses. And one of the things I do for one of my assessments, especially one of the assessments I engage my higher level students to come and rate the assignment and interact with students who are in the undergraduate level courses. “(Kelly, 4/15-23)

For Dan, the diversity of subject specializations is something that needs to be bridged even within departments. Dan explains:

"Yeah, absolutely. Yeah. I talked to a number of my colleagues. (My fellow faculty members) primarily about some of the stuff we do in our [Global Learning] classes. In fact I did a presentation. We have, we call it [the title of the event omitted by researcher] where a [faculty member] will present their research or what their subject specialization is. So I did one on global learning.” (Dan, 7/26-20)

The last examples of bridging diversity also coincide with another sub-theme. For many 
faculty members systematic change begins with moving global learning beyond the global learning classroom. The sub-theme of beyond the global learning classroom are instances where the global learning process has gone beyond the classes it was intended to change to other classes or contexts. Examples of the sub-theme of beyond the global learning classroom include this excerpt from Melissa who says about global learning engagement and course objectives, "I will be sharing it at this conference at the end of the year...” (Melissa, 9/10). Kelly adds:

"Yes. I have shared these experiences with my colleagues. But sure they have different courses. I shared the experiences of global learning workshops in my teaching with my colleagues. Some of the participation and discussion and even online discussion and all that which I have incorporated into this global learning course. Some of my colleagues have really liked those ideas or they have included or they have changed to some extent so their courses can have better outcomes.” (Kelly, 5/13-20)

Respondent: 27 says, "I think that the faculty involved in the GL workshops are like-minded in some ways. It's interesting to see global learning concept being applied to courses that I never thought of as being globally oriented” (Respondent: 27, 3/ 29-30). Respondent: 21in answering a question about how many global learning courses the faculty members teaches, makes this statement common amongst many faculty members about the informal use of global learning, "officially one -- but have incorporated aspects of global learning in my other courses also" (Respondent: 21, 3/38). Faculty members sometimes use global learning as a discussion point in career advancement. This respondent wrote, "I frequently share how I have continuing education in global learning 
in my CV and at interviews” (Respondent: 38, 3/23). FIU faculty members sometimes even try to bring what they learn home: "I shared the assignments with like-minded family/friends in hopes of assisting/supporting them in their process of transformation.”

Respondent: 10, 3/12-13)

\section{Summary}

This chapter presented the qualitative findings of this study. These findings show that FIU faculty who attended global learning professional development workshop and who reported transformational learning perceived this transformation as a series of individual and collective opportunities and challenges. The theme of individual opportunities included the sub-themes of revelation, clarity, negative transformation, and empowerment. The second theme of is of individual challenges. The theme of collective opportunities included the sub-themes of positioning, multidisciplinary communities and enthusiasm. The theme of collective challenges included the sub-themes of global and a call to leadership.

These FIU faculty members applied their transformation to their teaching by first exploring the theme of conceptual change and the related sub-themes of the importance of teaching, the goals of teaching, student centrism, and content and knowledge. The second way that FIU faculty members applied their transformation was through individual instructional change modeling learning resources and authentic global learning. The third way that FIU faculty members applied their transformation was by developing systematic awareness and the related subthemes of fulfilling the promise and support systems. The fourth way that FIU faculty members applied their transformation was through the theme of systematic change through instruction. The theme of systematic 
change through instruction was exemplified by the related sub-themes of bridging

diversity and moving beyond the global learning classroom.

\section{Overview of the Succeeding Chapter}

The next chapter of this study discusses the finding of this study, the implications

for practice, makes recommendations for research, explores the limitations of the study, and concludes this study. 


\section{CHAPTER VI}

\section{DISCUSSION}

This discussion is divided into four sections. First each research question and response is restated followed by a discussion of the responses to each research questions. Then there is a discussion of implications for practice. This is followed by recommendations for future research and a summary of the chapter.

The data from surveys and interviews were used to answer the research questions.

\section{Research Question One}

The first research question was: Do university faculty members who have attended a global learning professional development workshop report a transformational learning experience?

\section{Response to research question one}

The findings showed that several university faculty members who have attended a global learning professional development workshop reported having a transformational learning experience.

\section{Discussion of findings}

King (2002) argued that if the right needs are met for faculty, a professional development workshop can lead to transformational learning. The quantitative findings of this case study support the notion that transformational learning is possible in professional development and further suggest that professional development for global learning can also lead to transformational learning in university faculty members.

In the quantitative findings, there was a large amount of respondents who in some part identify as White or who teach in the College of Arts and Sciences. Florida 
International University has a high white population of faculty which is consistent with the university faculty member population of many other universities and in particular peer instittuions. In the Florida State University System (SUS), peer institutions are identified as institutions that are closely related in terms of the enrollment, diversity, and population data of a university. Peer institutions related to Florida International University include Florida Atlantic University (FAU), the University of Central Florida (UCF), and the University of South Florida (USF). The majority of tenured and tenure track faculty members in all these peer institutions are white and male. The majority of tenured and tenure track faculty members in other SUS institutions such as Florida State University (FSU) and the University of Florida (UF) are also white and male (HeydetKirsch \& Snellgrove, 2015). The majority of all faculty members in over 4,000 United States educational institutions (including public and private schools) are white and are male (Smith, Esau, \& Garcia, 2012).

FIU promotes global learning through undergraduate foundational and upper division courses (Green, 2013). The fact that a large number of survey responses were from faculty who teach in the College of Arts and Sciences is consistent with the fact that approximately over 57\% of Global Learning courses reside within the College of Arts and Sciences (Florida International University, 2015).

The quantitative findings not only answered the first research question but provided the foundation for the importance of the second and third research questions that are explored by this dissertation

\section{Research Question Two}

The second research question is: How do university faculty members who have 
attended global learning professional development workshops and report having transformational learning perceive global learning curricula as transformational learning?

\section{Response to research question two}

University faculty members who have attended global learning professional development workshops perceive global learning curricula as transformational learning occurring as individual opportunities, collective opportunities, individual challenges, and collective challenges. These themes were further identified as sub-themes. The theme of individual opportunities included the sub-themes of revelation, clarity, negative transformation, and empowerment. Examples of the second theme of individual challenges varied. These faculty members also saw this transformation as a series of collective opportunities and challenges. The theme of collective opportunities included the sub-themes of positioning, multidisciplinary communities and enthusiasm. The theme of collective challenges included the sub-themes of global and a call to leadership.

\section{Discussion of findings for research question two}

Mezirow (1998) allowed for four forms of critical self-reflection on assumptions. These four types of self-reflections are evident in the statements FIU faculty members made about how they were influenced by global learning professional development. Mezirow’s (1998) narrative critical self-reflection on assumptions is when the teacher is given a narrative, the teacher applies the narratives to himself or herself, as a problem, and then comes to a resolution. FIU faculty member "Dan" for example was given the narrative that the global learning process was a kind of top down indoctrination from administration, but he explored how the process applied to him, and came to the resolution that there was no indoctrination. 
"Some of the faculty I think were nervous that this was a top down imposition from administration but it was not at all. It was much more you know your ideas are driving the development of the syllabi and they were there basically you know to get you thinking about different aspects of how might the course come together and also incorporating global learning components.” (Dan, 1/41-45)

Mezirow’s (1998) systemic critical self-reflection on assumptions occurs when the teacher reflects on taken-for-granted cultural influences that may have to do with organization such as the work or social norms such as ethics. Some FIU faculty members noted that the FIU culture did not promote the importance of teaching and they also protested the popular conception of global learning at FIU as top down administration. These faculty did not give in to organizational or social norms. These faculty members felt it was their moral responsibility to protest and yet improve as teachers and continue with the development of their global learning courses.

Mezirow’s (1998) therapeutic critical self-reflection on assumptions occurs when one is examining one's problematic feelings and their related consequences. FIU faculty member Melissa for example had concerns about being able to create and modify her global learning courses. She realized her fears were tied to not understanding global learning or having support. Her reflection led to her going to the workshop to learn about global learning and seeking the enthusiastic support off global learning initiative staff members (Melissa, 1/38-39).

Mezirow’s (1998) epistemic critical self-reflection on assumptions is investigating the assumptions, the causes, the nature, and the consequences of one's frame of reference so as to surmise why one is predisposed to learn in a certain manner. Dan for example 
taught as a lecturer and was skeptical about rubrics. He reflected that this was the case because he himself had been taught in that manner, that is as a lecturer. Dan learned however that he could be a better teacher by employing the team learning, active learning, and assessment strategies presented to him at the global learning professional development workshops. (Dan, 2/43-3/3, 3/24-31, 7/3-9)

In the findings of this study, faculty members at FIU began to identify themselves in comparison to other faculty members in terms of their belief in global learning (Dan, 1/41-45; Frank, 1/26-33; Respondent: 4, 3/11-12). The enthusiasm of the true believers in global learning also seems to have a positive influence on other faculty members (Respondent: 22, 4/ 3-5). Many faculty members felt empowered and more engaged after dealing with the enthusiasm of those who promote global learning at FIU (Melissa, 1/3839; Respondent: 22, 4/ 3-5). These findings are in line with the work of Stohl (2007) who argued that the chief challenge for developing and sustaining internationalization in the context of the challenges and opportunities of the 21st century is the engagement of the faculty. Childress (2010) also argued that internationalizing the curricula is not possible if the faculty do not have the knowledge, skills, attitudes, and institutional support to do so, and identifies five essential components in a model called the Five I's of Faculty Engagement in Internationalization (Intentionality, Investments, Infrastructure, Institutional Networks, and Individual Support). Arguably, FIU faculty members could already be disposed towards global learning because their institution has tried to fulfill these components.

Green (2007) observed the role of faculty in internationalizing the curricula after researching the work of community colleges. She pointed out that successful programs 
have faculty development programs, have point people in charge of helping faculty, have faculty members with a global mindset, and place the student at the center of learning. Through the global learning professional development workshops and the larger global learning initiative, faculty members at FIU could be said to have instituted these roles to the advantage of the institution.

The sub-theme of positioning occurs in the findings of this case study where the FIU faculty members are able to find their individual and collective identity by their degree of belief in the mission of global learning. This parallels the categorizations Childress (2010) has developed. Childress argued that individuals and teams and institutions have to work together in an integrative holistic process. Different levels of faculty engagement are categorized under the headings of champions, advocates, latent champions and advocates, uninterested, skeptics, opponents. The findings of this dissertation suggests that collectively FIU faculty members in all categories work to make the global learning process successful despite of and perhaps even with the benefit of the skepticism and/or protest of several individual faculty members who engage in critical reflection and social discourse and may improve the process with their insights and actions. It is clear in the findings that many individual faculty members became interested in and/ or became advocates for global learning in part through their interactions with the champions of global learning at FIU.

A major factor in how successful a faculty member is with changing the curricula of their content area is if they were taught that content with an eye towards how much knowledge can be culture bound (Green, 2007). For a faculty member who has not learned how much of what they know about their subject is culturally bound, it could be 
difficult to design a course that reflects that their content, how it is taught, and viewed is open to many different perspectives, interpretations, and could be made to reflect global learning.

FIU promotes team based learning and teaching for students in global learning classrooms (Green, 2013). This means that students of different abilities and skills sets work together to solve global problems and learn content in a classroom. In order for that environment in a classroom to exist faculty members first learn to work as teams at the workshops and in particular to learn to solve the problem of creating or modifying the global learning courses they will teach.

The opportunity to have the global learning initiative staff and other colleagues in a multidisciplinary community at the workshop results in a positioning by the faculty members which can contribute significantly to their understanding how knowledge can be viewed in different ways, how it can be taught in different ways, how it can cross cultural boundaries, become globalized, and become part of a global learning course.

Faculty members who had worked abroad felt they had insights into the importance of global learning for students (Respondent: 14, 1/27-35). Faculty who presented with a long established international mindset can draw on the resources of other settings, cultures, and languages and are more adept in translating, synthesizing, integrating, and connecting their knowledge (Green, 2007). As FIU faculty member Kelly noted, the workshops afforded an ecology of experience, a gateway to establish multidisciplinary communities of scholars, and a chance to watch faculty members of various backgrounds tackle problems such as how to view and handle knowledge to be used in a global learning course (Kelly, 5/27-21). Arguably the workshops and the 
relationships established there helped individual FIU faculty members with varying degrees of an international mindset reach a higher collective bar in terms of that mindset.

For many FIU faculty members, the workshops were very important because it was an opportunity to interact with other faculty members (Dan, 2/24-32; Kelly, 2/1-2/7). The ability to interact face to face with faculty from different parts of the university that individual faculty members usually do not have an opportunity to interact with creates an environment for social learning. According to Bandura (1977), social learning is basically learning by observing the behaviors of other with the environment in which learning happens being a factor along with cognition. For learning to occur, an individual must observe and remember the behavior, must be able to imitate the behavior, and must have a reason to learn the behavior.

Several of the FIU faculty members stated the importance of workshop staff and their availability and close proximity (Melissa, 1/38-39; Respondent: 22, 4/ 3-5). Arguably they were able to model their role as teacher in a future global learning classroom upon the behavior of the workshop staff member they observed. Many of the FIU faculty mentioned in some way the importance of working together with other faculty members. Since a global learning classroom is meant to be dialogic, faculty members could also observe and perform a variation of the role of the global learning student by working as part of a team in the work shop to solve the problem of putting a global dimension into their courses. This kind of social learning gave them an insight into both the role of educator and student in the classroom and brought an abstract idea (creating or modifying a global learning course) briefly into a real environment in which they could interact (a variation of the team based, active learning classroom mimicked in 
the workshop). Importantly, FIU faculty had several motivations for learning these behaviors, including but not limited to the need to create the course, the convenience of working with others, the desire to have a good assessment of their teaching by global learning staff ,students, and other FIU staff, and a need to do what they felt was best for their students.

Social learning in and beyond the workshops can help explain how FIU faculty members experience a sense of individual and collective challenge. In the findings, the sense of challenge may have been difficult but it was not found to be stifling. The challenge was often seen as an ongoing process and not just one swift defining action. This conception of challenges implies that sooner or later the challenge could be met by university faculty members. Successful social learning promotes a sense of self efficacy in the people who experience it (Bandura, 1977).The opportunities FIU faculty members encountered particularly those dealing with emotional energy such as a sense of empowerment may have softened the challenges FIU faculty members encountered by creating a sense of self efficacy.

For many faculty members, it was important for them to establish their own position before discussing their thoughts on the global learning process. This is arguably a kind of perspective consciousness that Hanvey's (1975) global perspective promotes. With perspective consciousness, a global learner learns and/or becomes aware that they have a perspective on something and that others have their own perspectives on the same thing. Arguably for faculty members there was an awareness of their perspective on global learning that emerged as they became aware of other faculty members' perspectives on global learning. As they both learned about the global learning process, 
Dan found the process much more fluid and developing from the ground up than did Frank (Dan, 1/41-45; Frank, 1/26-33).

Many FIU faculty members took on a positive perspective of global learning (Dan, 1/41-45; Martin, 8/7-21). Other faculty members took a negative perspective on global learning that led to a kind of negative transformation (Respondent: 6; Respondent: 13). It is possible that a respondent protested but they were not a part of the qualitative stage of this study because they did not meet the criteria for participation in the interviews. Respondent: 131.94.186.73 for example had a survey that was labeled as nontransformational learning by the researcher which included a clear statement that disagreed with the global learning process. It is also impossible to know if those who had not completed a survey had disagreements with the global learning process.

Mezirow (2000) noted that people can change their points of view by exploring another person's point of view but they cannot try on someone else's habit of mind. Discourse in the workshops helped change perspectives on global learning for FIU faculty members. However while many of them could have agreed on a particular perspective these faculty members may have had habits of mind that helped them reach that same particular perspective, whether negative or positive. This is something that is exemplified in the sub-theme of negative transformation where several faculty members were in agreement in their protest of global learning but had different mindsets as to why they were protesting global learning (Frank, 1/19-21; Respondent: 13, 2/15-17;

Respondent: 6, 1/21-24, 2/16-17; Respondent: 27, 2/25).

\section{Research Question Three}

The third main research question asked: How do university faculty members who 
have attended a global learning professional development workshop and report having had a transformational learning experience apply this transformation to their teaching of global learning curricula?

\section{Responsive themes to research question three}

University faculty members apply their transformation to their teaching by collectively developing concepts that inform individual instructional change, and then creating systematic awareness and attempting systematic change through their pursuit and/or application of instructional change. The major themes in response to research question three are conceptual change, individual instructional change, systematic awareness and systematic change through instructional change. These FIU faculty members applied their transformation to their teaching by first addressing issues of conceptual change and issues in the related sub-themes of student centrism, content and knowledge. The second way that FIU faculty members apply their transformation is through individual instructional change as illustrated in the sub-themes of modeling learning resources and authentic global learning. The third way that FIU faculty members apply their transformation is by developing systematic awareness and exploring the related sub-themes of fulfilling the promise and support systems. The fourth way that FIU faculty members apply their transformation is systematic change through instruction and the related sub-themes of bridging diversity and moving beyond the global learning classroom.

\section{Discussion of findings for research question three.}

Many of these faculty members who had a transformational learning experience discussed moving from teaching strategies centered on faculty expertise such as lecturing 
to strategies centered on the students being active in their own learning (Respondent: 76.111.197.127, 2/33-36; Respondent: 18, 3/5-6). Holden (2000) promotes the use of active learning in the instruction of global learning. As noted by Greene, FIU both uses and promotes active learning strategies in global learning workshops (2013). Active learning strategies allow students to go beyond learning by passive listening to learning by actively working on problems and issues as part of their own teaching and learning process (Bonwell \& Eison, 1991).

Many of these faculty members discussed how their strategies allowed students to take on the educator role as well as the learner role which in turn also allowed faculty members to see the world the way their students do (Respondent: 26, 1/35; Respondent: 27, 2/2-5; Respondent: 22, 2/ 4-6). Faculty members also noted the importance of this type of instruction because they began to see their students as actors and change agents in the world (Respondent: 26,1/35) and as well saw the importance of global citizenship for faculty members as well as students (Respondent: 22, 1/ 17-20). While FIU's use of active learning strategies in part can explain the fact that becoming more student centered and moving beyond lectures was commonly brought up by faculty members in this study, it does not fully explain the importance of related statements in the data where FIU faculty members describe trying to form a partnership with students (Respondent: 13, $1 / 35$ ) or statements that describe students and faculty members both growing into better global citizens (Respondent: 22, 1/ 17-20). Friere (1971) suggests that education is political and those in power promote a kind of banking education where official knowledge is imputed in a monologic way into students whose own voices are rendered powerless. The lecture mode many faculty members at FIU used in the past is a display 
of this kind of power. Moving from the lecture style and dialoguing with students, finding out their perspectives, and making them active participants so that students can code and decode their world is a change in the power structure of education. Faculty members who formed a partnership with students (Respondent: 13,1/35) imply a negotiation of power. Statements that describe students and faculty members growing into better global citizens (Respondent: 22, 1/ 17-20) imply a larger political transformation for both faculty and students. Statements in the sub-theme of negative transformation where faculty members were highly critical of the imposition of global learning on them and students (Frank, 1/19-21; Respondent: 13, 2/15-17; Respondent: 6, 1/21-24, 2/16-17) suggests a power struggle among faculty; that is for some faculty members the promotion of global learning is a display of power over students, and that teaching is a political act.

Faculty members came to understand the importance of teaching (Respondent: 22, 1/ 17-20; Respondent: 26, 1/17; Respondent: 22, 1/ 17-20) in this study and noted that while the Office of Global Learning Initiatives promotes the importance of teaching (Respondent: 20, 2/1-6; Respondent: 22, 2/16-18), the university does not have a reward system to support focusing on teaching as a way to move forward career-wise (Frank, 2/3-14).

Cranston (1996) explored perspectives that drive professional development to explain how adult learners become transformational learners. The humanist perspective on professional development looks at teaching as primarily nurturing and developing learning in students who once educated use the knowledge they gain in any way the students see fit. The social activist perspective looks at professional development as a political act. Faculty members must balance the multiple roles of educator, learner, and 
activist within the social activist adult learners' identity effectively. The findings seems to support the notion that professional development at FIU is closer to a humanist perspective than it is a social activist perspective. While the work of Freire (1971) informs the transformational learning of Mezirow (2000), the findings do not demonstrate the kind of high levels of critical awareness that liberates the faculty members although the workshops seem to empower them. The global learning classroom these faculty members create may also be venues for critical awareness but the findings suggest that the classroom can lead to transformation but are not as politically emancipatory as the conception of transformational learning Freire (1971) promoted.

Faculty member Dan explained how faculty members use lectures because they themselves were taught that way. The workshops inspired him to question this way of teaching (Dan, 2/43-3/3). The work of Habermas (1971) on forms of learning also informs the work of Mezirow (2000). Habermas suggests that practical learning is based on social norms while instrumental learning is based on reflection. It is possible that social norms may have had a hand in the promotion of passive learning strategies by FIU faculty members who taught in the way they themselves were taught and were expected to teach in the past. Global learning may have brought the reflection needed to transform towards more instrumental learning. Faculty members also helped each other through discourse, collaboration and the sharing of ideas (Kelly, 1/22-25). Habermas (1971) also promoted the need for discourse and communication of the type promoted in the workshops and needed for Mezirow’s (2000) transformational learning. 
Faculty members work with many forms of diversity within their classroom to address many problems and issues (Respondent: 22, 1/ 30-35; Martin, 14/30-14/41). Hovland and Musil (2005) argued that global learning can inform U.S. diversity education and its global contexts, bring attention to segments of the academy that are marginalized and ignored, and revise our basic understanding of what we need to know about questions of race, class, gender, religion, sexuality, ethnicity, and other forms of diversity that have profoundly transformed higher education (Hovland \& Musil, 2005). From the findings of the case study, it is apparent that the faculty members feel that they are making their classrooms a forum to allow for this kind of discourse to be centered on the diversity of the learners. It is not clear that the discourses in the workshop discussed thoroughly how these issues occur in the global learning ecology of which the faculty members are a part. For example, some FIU faculty discussed the Western centrism of global learning (Respondent: 13, 2/15-17) but there was no statement about how the global learning process and gender issues amongst faculty could influence each other.

In the global learning literature, it is said that internationalizing the curricula can also help in promoting multiculturalism and international studies (Shultz, SkiltonSylvester, \& Shultz, 2007). Towards this end, the findings showed a commitment by FIU faculty members to bridge diversity in making connections with their students and to use international case studies in their teaching (Martin, 4/38-45).

Faculty members discussed how the workshop promoted and explored how others view and solve problems, and modeled this work (Melissa, 2/22-29). While many strategies can be called active learning strategies, problem based learning coupled with critical thinking can be particularly effective in the retention of learning by students 
(Lamy, 2007). The fact that the workshops presented problems for the individual faculty member to deal with meant that active learning strategies were not only taught but performed, and that the workshops were venues for critical reflection and social discourse. This may have helped the faculty members learn and retain their knowledge better than if they were taught in a different much more passive way.

Several faculty members reflected on how the approach to curricula design in the workshop promoted made them rethink the way they looked at their work (Dan, 4/45-5/2; Melissa, 4/33-41). FIU employs backwards curriculum design (Green, 2013). Backwards curriculum design starts with what the student outcomes should be and how they can be assessed and ends with developing the content and instruction that leads to those outcomes (Wiggins and McTighe, 2005). As evidenced by the sub-themes that focused on the goals of teaching and what was taught and how I was taught, this backwards curriculum design process helped disrupt the hegemony of traditional teaching for FIU faculty members, making them focus more critically on all the elements of course design, and reflect on why they were doing what they were doing.

Faculty members learned by interacting with each other (Kelly, 1/22-25) and the staff of the Office of Global Learning Initiatives (Melissa, 9/19-24). FIU uses dialogue between the Office of Global Learning Initiatives and faculty members to help keep them continuously engaged. However the staff of this office is relatively small, the budget is limited, continued existence far beyond accreditation is uncertain, and there is a growth in the number of faculty involved in global learning (Green, 2013). What may seem as a looming problem may however work as an opportunity since faculty members are developing relationships amongst themselves that are helpful and these can 
counterbalance the strain on the staff members of the Office of Global Learning Initiatives. When the number of faculty members engaged in global learning exceeds the support of the OGLI staff, FIU should have an environment where faculty members new to global learning can turn to a community of faculty members who have a long experience with global learning. This would also to some extent give the larger community of scholars at FIU a more hands on opportunity to shape the continued global learning dialogue and process at FIU.

The way FIU faculty members seek to fulfill what they perceived to be the promise and unique mission of FIU and to make systematic change related to the university (Martin, 8/30-9/11; Respondent: 24, 3/17; etc.) could be supported by Musil (2006). He argued that institutions have to be more intentional in promoting a process through which key stakeholders in global learning can reflect deeply on what the institution wants to achieve through its commitment to global education, what niche the institutions fulfill in the global learning process, and what in that particular university culture informs the global learning process. One issue for FIU faculty for example is the desire for making Florida International University a truly international and global university.

Student centrism was a major focus for many of the FIU faculty members. There were several statements in this case study where faculty members had felt they had improved as teachers and had improved student learning by letting students become an active part in their own instruction (Respondent: 14, 1/27-35; Respondent: 14, 3/3; Respondent: 18, 3/5-6). The importance of students as a resource and inspiration and the need for rewards, incentives, and funding for faculty has been noted by Childress (2010). 
Stohl (2007) argued that to capture the faculty’s interest in, and commitment to, internationalization, stakeholders need to move beyond the conceptualization of the internationalization or globalization of higher education in terms of how the different aspects of teaching, research, and service functions of the university are becoming more internationalized and go further by examining how these activities encourage greater learning and discovery. However the challenge is to convince faculty that their scholarship and teaching will benefit from these efforts by considering the risk and reward structures within their institutions and faculty cultures (Stohl, 2007). In FIU, many faculty members become more engaged in the global learning process when they begin to see how it transforms the learning of their students. The reward for them is the improvement of teaching and learning (Respondent: 24, 1/ 38-39; Respondent: 10, 3/1213).

Many FIU faculty members saw a need to add greater study abroad opportunities as a resource along with the internationalized curricula (Martin, 8/30-9/11; Melissa, 1/3336). With respect to these opportunities Stohl (2007) argued that although higher education has been successful in providing mechanisms for student mobility, internationalization often fails because institutions have not successfully engaged the faculty. Even if student mobility is not as sizeable as it should be it does not in and of itself, deliver the learning, discovery, and engagement that everyone seeks through internationalization. Some faculty perceive that FIU's faculty engagement and internationalized curricula have progressed more than study abroad (Martin, 8/30-9/11; Melissa, 1/33-36). 
Faculty members also expressed the need to improve study abroad (Martin, 8/309/11; Melissa, 1/33-36). Green \& Schoenberg (2006) contended that because the vast majority of U.S. students do not study abroad, and many of these students commute to campus and have families and jobs that keep them from attending campus events, the major venue for global learning is in the classroom. And even for those students who do go abroad or attend campus events, the curricula is still the foundation of a college education. Woodruff and Henry (2012) argued for curriculum integration because study abroad programs or other facets of a university do not always have an internationalized curriculum that is in support of the larger aim of internationalization. Both Ellingboe (1998) and Harari (1992) have contended that faculty are critical to the process of internationalization of the curriculum because they have direct responsibility over curriculum content and its teaching and can model how much they value it to their students. For some FIU faculty to have the kind of study abroad program they want, there has to be an organizational commitment to make study abroad more practical, affordable, and available to students, and a commitment to incentives to engaging faculty but more importantly a commitment to internationalize the curricula of all study abroad classes (Martin, 8/30-9/11; Melissa, 1/33-36). FIU faculty members seem to be far from that goal of systematic change as the findings of this case study imply.

Through the learner centered approaches (Respondent: 76.111.197.127, 2/33-36; Respondent: 27, 2/15) and the multidisciplinary communities made available to FIU faculty members through their interactions in the workshops (Kelly, 2/1-2/7; Respondent: 26. 3/9), FIU faculty have an opportunity over time to create networks that can encourage the development of knowledge and skills so as to promote multidisciplinary, multilevel 
and multicultural approaches in research and education as suggested by Marsella (2007). Marsella insists that adequate training involving multidisciplinary methods, distance learning, new technologies, as well as requiring knowledge of global modeling, global communities and extensive field research are necessary for this process. This is not something mentioned in any of the findings of this study.

Rauch and Steiner (2006) argued that the type of critical approach used in global learning presupposes a set of specific competencies in systems thinking, team skills, the ability to deal with insecurity, integrated thinking, the readiness to cooperate, coping with change, creative and lateral thinking, and readiness to compromise. The findings of this case study, where faculty members are making individual and conceptually centered instructional change and appropriating tools and strategies towards the end of creating systematic change, suggests that at least some of these competencies are mastered by FIU faculty through their interactions with the global learning process at FIU.

Mezirow (1985) described a process of instrumental, dialogic, and self-reflective learning where learners could ask how best to learn knowledge (instrumental), what environment or context is best for learning (dialogic), and why they are learning (selfreflective). Arguably, the global learning professional development workshop helps lead FIU faculty members through a variation of this process. In terms of instrumental learning, the faculty member asks how their students could learn their discipline best in teaching the discipline in a global learning course. In terms of dialogic learning, the faculty members could decide on the best method of teaching their subject in a global learning course by selecting an active learning method through the context of a dialog with other faculty members and global learning initiative staff members at the workshop. 
Finally, the faculty member becomes a self-reflective learner by observing why they learn by, for example, seeing that one active learning strategy is doing better at raising assessment scores of their students in their classes than another and then making modifications based on those observations to improve instruction.

This process of instrumental, dialogic, and self-reflective learning can internalize itself in the transformation of the faculty member and also can scaffold a schema for making systematic change, transforming the university, communities, and the world beyond. Instrumentally, a faculty member learns about the success of applying global learning to their classroom and attempts to apply it beyond the classroom, dialogically the faculty member talks to colleagues and others to find methods to apply global learning to other aspects of their world, and self reflectively the faculty member sees what works and what doesn't to change the system and makes modifications. In the case of FIU faculty member Dan for example, he witnessed the success of the global learning process in his course work, he promoted awareness and discussion about global learning with colleagues who had not attended the workshops and came up with methods to promote it in the systems with which he interacts, and then through a process of trial and error came up with several projects to create systematic change at the university that was successful (Dan/7/20-39). When Dan shares these insights and works with and inspires others he goes from being an individual change agent to being a collective change agent.

\section{Implications for Practice}

At the institutional level it is recommended for practice that FIU use the work of the OGLI as a model for professional development throughout the university. It is also recommended that the OGLI at FIU focus on transformational learning on a regular basis. 
It is recommended that the OGLI at FIU adopt the survey and/or interview instruments for future use in evaluating their work with faculty members. The data collected could reveal what are the actual practices of faculty and how to improve these practices. Exploring these practices would also address gaps in the global learning literature.

In the findings, it was clear that transformed FIU faculty members who participated in the study, who teach or have taught a global learning class, had multiple opportunities for professional development, were demonstrated various pedagogical tools, strategies, and resources, and were given a supportive environment. These are all in line with the factors researched by Navarro (2004) as factors which affect participation of faculty in the internationalization of the curricula. It is important that other institutions consider adopting these practices so as to promote faculty engagement through transformation of faculty members.

Dewey and Duff (2009) stated that although faculty are necessarily key participants in initiatives to internationalize academia, surprisingly little work has been published that addresses the roles, responsibilities, and problems faced by the faculty on an operational level. The findings of this study add some insight into what faculty members are doing and could be doing in relation to internationalization. One issue is clear, ongoing dialogue about global learning and internationalizing the curricula is important for practice so as not to limit the dialogue to a smaller group of key stakeholders at the university or limit the forms of input by the university as a whole. By the time many of the FIU faculty members enter a workshop much of the conversation on global learning as it relates to the faculty and students has already taken place. Adding to the tension is the fact that as the findings of the workshops revealed, demystified, or 
clarified a conception of global learning, the FIU faculty members could have had different views and/or changed those views. Consequently whether or not FIU faculty members agreed with the goals of global learning, it was important for them and for the university, the teaching profession, and for the success of the professional development, that these faculty members have a forum for airing grievances, making protests, giving constructive feedback, adding insights, coming to acceptance or making compromises in relation to internationalization of the curriculum and global learning.

\section{Recommendations for Future Study}

It is recommended that there should be longitudinal follow up studies in the institution of FIU on how the transformational experiences of faculty participating in global learning professional developments workshops inform their practice over the long term.

Follow up studies on faculty members who experienced transformational learning after participating in global learning professional development workshops should be done using other methods. These follow up studies should use classroom observations judged with rubrics and checklists in order to ascertain how practices have been informed by global learning and professional development in a way that goes beyond the selfreporting done in the case study of this dissertation.

It is also recommended that future studies interview faculty members whose surveys can be categorized as having non-transformational learning or only partial transformational learning. This should be done in order to better understand why these faculty members think the way that these faculty members do.

Because this study was focused on one university, there were a limited number of 
participants with special characteristics that may be specific to Florida International University. Future researchers should attempt a study where the purpose is to explore how global learning is transformative for faculty members across several universities and how faculty members apply this transformation to their instruction. The sharing of findings may be important for sister universities linked by geography or similar goals and expectations such as state universities.

The recent wave of interest in and implementation of global learning has promulgated further and deeper discussion about the function of study abroad. In particular it has been posited that universities move from just relying on study abroad and instead or in addition use cultural interactions in their home communities and create a campus wide global learning ecology. For various institutions this had been a challenge due to their particular circumstances (Hovland, 2005). The creation of a global learning environment in a very homogenous context means that the experience of students learning in the community is less advantageous than in other universities. This was the case at the University of North Carolina Chapel Hill as described by Smith and Kruse (2009). Because of the culturally and ethnically diverse student population of FIU this could indicate that FIU might be able to create such a global learning ecology. It is recommended that future research should explore if there is a difference in faculty transformation when a similar global learning process is used across different environments with different levels of homogeneity and how instruction is affected.

FIU has a more diverse population on and off the campus and it is situated in the large cosmopolitan city of Miami. However opportunities for students to learn about other cultures face to face in Miami are made problematic by enclave cultures and 
commuter centers where people can literally drive past different cultures in the isolation of their vehicles (Landorf, Rocco, \& Nevin, 2006). Future studies could look at not just the universities themselves but include the communities in which they are situated to examine if the inclusion of those different local communities as part of a global learning curricula changes how faculty members are transformed and how they apply their transformation to their teaching.

Schenck (2005) noted that global learning in less homogenous contexts with cultures that have histories of violence against each other sometimes creates highly charged environments where discussions can quickly become heated and lead to psychic and/or physical harm if the situation is not diffused. As such conflict resolution in the classroom can be as important as learning about cultures and languages. Missing in this case study is any detailed sense that the development of conflict resolution strategies and resources were needed or used by FIU faculty in order to improve instruction and change the system. Future research should look at the importance of conflict resolution in the promotion of global learning. Conflict should also be explored not just amongst the students in a classroom but amongst the other stakeholders in the global learning ecology such as the faculty members. Future research should explore the question of whether conflict resolution was needed to promote global learning in the classroom and if so, did it lead to transformation for students or faculty members.

Stohl (2007) argued that the reward of better student learning and improved scholarship can inspire more faculty engagement despite the many obstacles of internationalization. The findings at FIU only show that improved student learning is an incentive for transformed faculty members to be engaged in their work. The findings of 
this case study show that transformed faculty members take advantage of the multidisciplinary communities of faculty members that develop because of the workshops and these faculty members also learn from interacting with their students to improve their global learning courses. The findings however suggest that faculty members may see opportunities to improve their scholarship but there is little evidence to show that it actually happens. This may be a function of this case study which is much more focused on instruction and practice and not research and scholarship. It is recommended then that future scholarship explore the idea of whether the promotion of global learning at the university has led directly to completed faculty multidisciplinary research. If global learning has led directly to completed multidisciplinary research, a study should explore how this manifested itself in order to see how to best promote this concept. Future study may also explore if this multidisciplinary research has had an impact on instruction. Future study should also seek to explore if global learning is a significant factor in the promotion of multidisciplinary faculty research.

\section{Limitations of the Study}

A limitation of this study could be the limited amount of faculty members that participated in the interviews following the surveys. Future work should include interviews with a larger number of faculty members.

The setting for the study is a metropolitan and multicultural area where the university is located which may naturally create interactions similar to global learning amongst the student body that may be less likely in a less dynamic and more monocultural setting. This could mean that students come into the class with more practice in global learning. This could also mean that if students come to a global 
learning course without any experience in global learning, they could have more opportunities to experience the process of global learning at this university because the environment of the school is more multicultural than that of other institutions in other parts of the United States. This university is also a research institution with many resources that could be used for global learning that may not be available at smaller and less funded institutions.

This study may also be limited in a way similar to a problem Hett (1993) encountered during the development of the Global-mindedness Scale. The problem for Hett was that students could have already been primed to be global minded through exposure to the media because of the unusually political nature of the era in which the instrument was developed and used (Hett, 1993). The study conducted here comes at a time when social media and the political activism of young people throughout the world are at a high point. The results obtained in this study could vary from year to year depending on the extent of the interest, activism and the political knowledge and interaction of the faculty participants and what they are teaching and discussion in the classrooms. No account was taken for this potential limitation but the political discourse of the time could have affected university faculty members.

\section{Summary}

This chapter concludes this study. This chapter presented a discussion of the findings of the study, implications for future practice, and recommendations for future research. 


\section{REFERENCES}

American Council on Education. (2003). Global learning. Washington, DC: Author (http://www.acenet.org/international/)

Anderson, J. L., Levis-Fitzgerald, $M$ R., and Rhoads, R. A. (2003). Democratic Learning and Global Citizenship: The Contribution of One-Unit Seminars. The Journal of General Education, 52(2), pp. 84-107.

Appiah, K. A. (2010, April). World citizenship. The life of the mind series. Lecture presented at Florida International University; Miami, FL.

Bandura, A. (1977). Social Learning Theory. General Learning Press.

Barbiero, V.K. (2008) Global Health for Undergraduates. American Journal of Preventive Medicine. 35.3 : p269.

Bartell, M. (2003) Internationalization of universities: A university culture-based framework. Higher Education, 45: 43-70.

Bertalanffy, L. V. (1950). An outline of general system theory. The British Journal for the Philosophy of Science, 1(2), 134-165.

Bonwell, C. C., \& Eison, J. A. (1991). Active learning: Creating excitement in the classroom. Retrieved from ERIC database. (ED336049)

Boyatzis, R. (1998). Transforming qualitative information. Thousand Oaks, CA: Sage.

Brewer, E., \& Leask, B. (2013). Internationalization of the curriculum. In D.K.Deardorff,H. de Wit, J.D. Heyl, \& T. Adams, (Eds.) (2013). The Sage handbook of international higher education (pp. 245-267). New York, NY: Sage.

Brock, S.E. (2010). Measuring the Importance of Precursor Steps to Transformative Learning. Adult Education Quarterly. 60(2) 122-142.

Brookfield, S. (1995). Becoming a critically reflective teacher. San Francisco, CA: Jossey-Bass.

Brookfied, S,D. (2002) Using the Lenses of Critically Reflective Teaching in the Community College Classroom, New Directions for Community Colleges, no. 118, (31-38).

Caffarella, R.S. and Zinn, L.F. (1999). Professional Development for Faculty: A Conceptual Framework of Barriers and Supports . Innovative Higher Education, 23(4), 241-254. 
Caffarella, R. S. (1994). Planning programs for adult learners. A practical guide for educators, trainers, and staff developers. San Francisco: Jossey-Bass.

Caruana, V, (2011) Preservice Teachers' Perceptions of their Perspective Transformations: A Case Study" . An unpublished dissertation. University of South Florida: Tampa. $T$ http://scholarcommons.usf.edu/etd/3034

Castells, M. (1999). Information technology, globalization, and social development. Geneva, Switzerland: United Nations Research Institute for Social Development. Retrieved from http://vega.soi.city.ac.uk/ abct353/SM1061N/Castells_1999.pdf

Coelen, R.J. (2009). Ranking and the measurement of success in internationalisation: are they related? In de Wit, H. (ed.) (2009). Measuring success in the internationalisation of higher education: EAIE Occasional Paper 22.

Amsterdam: European Association for International Education (EAIE). 39-48.

Cornwell, G. H., and E. W. Stoddard. (1999). Globalizing knowledge: Connecting International and intercultural studies. Washington, DC: Association of American Colleges and Universities.

Cummins, J. and Sayers, D. (1997). Brave new schools: challenging cultural illiteracy through global learning networks. New York: St. Martin's Press.

Camblin Jr., L., and Steger, J.A. (2000). Rethinking faculty development. Higher Education 39(1), 1-18.

Cushner, K. (2007). The Role of Experience in the Making of Internationally-Minded Teachers. Teacher Education Quarterly. pp. 27-39.

Dahl, T. (2009). The Importance of Place for Learning about Peace: Residential Summer Camps as Transformative Thinking Spaces. Journal of Peace Education, 6(2), 225-245.

Daloz, L. (1986). Effective teaching and mentoring: Realizing the transformational power of adult learning experiences. San Francisco: Jossey-Bass.

Davies, L. (2006). Global citizenship; abstraction or framework for action? EducationalReview, 58(1). 5-25.

Deardorff, D. K. (2004). The identification and assessment of intercultural competence as a student outcome of international education at institutions of higher education in the United States. Unpublished dissertation, North Carolina State University, Raleigh.

Deardorff, D.K. (2011). Assessing Intercultural competence. New Directions For Institutional Research, 149, pp 65-79. 
Deardorff, D., Pysarchik, D.T., and Yun, Z. (2009). Towards effective international learning assessment: principles, design and implementation. In de Wit, H. (Ed.) (2009). Measuring success in the internationalisation of higher education: EAIE Occasional Paper 22. Amsterdam: European Association for International Education (EAIE). 23-38.

DeSanctis, G., Wright, M., and Jiang, L. (2001). Building a global learning community. Communications Of The ACM. 44(12), 80-82.

de Wit, H. (2002). Internalization of Higher Education in the United States of America and Europe: A Historical Comparative, and Conceptual Analysis. Westport,CT: Greenwood Press.

de Wit, H. (2009). Measuring success in the internationalisation of higher education: an introduction. In de Wit, H. (ed.) (2009). Measuring success in the internationalisation of higher education: EAIE Occasional Paper 22.Amsterdam: European Association for International Education (EAIE). pp 1-8.

Doscher, S.P. (2011). The Development of Rubrics to Measure Undergraduate Students' Global Awareness and Global Perspective: A Validity Study. Ed. D. thesis, Florida International University. ProQuest (AAT 3517005).

Drucker, P. (1999). Beyond the information revolution. Atlantic Monthly, 284(3), 47-48.

Duerst-Lahti, G. (2005). Recasting Religious Studies at Beloit College Diversity Digest. 8(3) pp. 2-3.

Espiritu, K.M. (2010). Campus internationalization initiatives: From policy to practice in study abroad programming. Dissertation Abstracts International Section A: Humanities and Social Sciences. 2010, pp. 2316.

Eley, M.G. (2006). Teachers' conceptions of teaching, and the making of specific decisions in planning to teach. Higher Education, 51: 191-214

Elrod, S. and Hovland, K. (2011). Global learning and scientific literacy at the crossroads. Diversity \& Democracy. 4(2), pp. 1,3.

Fantini, A. (2009). Assessing Intercultural Competence: Issues and Tools. In D. K. Deardorff (ed.) (2009). The SAGE Handbook of Intercultural Competence. Thousand Oaks, CA Sage.

Fereday, J. and Muir-Cochrane, E. (2006). Demonstrating Rigor Using Thematic Analysis: A Hybrid Approach of Inductive and Deductive Coding and Theme Development. International Journal of Qualitative Methods, 5(1), 80-92.

Florida Administrative Rule. (1976). 6C8-1.001 Purpose. Tallahassee, 1976. 
Florida International University (n/d). Mission and Vision. Retrieved from http://www.fiu.edu/about-us/vision-mission/index.html

Florida International University. (1974). International...It's Our Middle Name. PressRelease. Jan. 1974.

http://qep.fiu.edu/QEP_Report/Report/International_Its_Our_Middle_Name.pdf

Florida International University. (2010). Florida International University’s quality enhancement plan global learning for global citizenship. Retrieved fromhttp://goglobal.fiu.edu/QEP_Report-Final.pdf

Florida International University (2012a). FIU Fast Facts 2012. Retrieved from:http://opir.fiu.edu/factbook.htm

Florida International University (2012b). Employee data. Retrieved from:http://opir.fiu.edu/factbook.htm

Florida International University (2012c). Global Learning for Global Citizenship Annual QEP Impact Report, 2011-12. Retrieved from:http:/goglobal.fiu.edu/About/Pages/Impact-Reports.aspx

Florida International University. (2012-2013). Graduate Catalog. Retrieved from:http://catalog.fiu.edu/2012_2013/Graduate/Admissions_and_Registration Information/

Florida International University. (2014). Factbook. Office of Planning and Institutional research.http://opir.fiu.edu/factbook.htm

Florida International University. (2015). Office of Global Learning Initiatives. Global Learning courses. http://goglobal.fiu.edu/courses.

Freeman, R. E. (1993) Collaboration, global perspectives, and teacher education. Theory into Practice, 32 (1). 33-39.

Freeman, I., and Knight, P. (2007). Fostering Global Perspectives in Undergraduate Marketing Students- A Kazakhstani/Canadian Collaboration. Canadian Journal of Higher Education. 37(2), 47-67.

Freire, P. (1970). Pedagogy of the oppressed. New York, NY: Seabury Press.

Geelhoed, G.W. (2009). Transformational learning in health care students through international service in developing world environments. George Washington University.

Gibson, K. L., Rimmington, G. M., \& Landwehr-Brown, M. (2008). Developing global awareness and responsible world citizenship with global learning. Roeper Review, 30(1),11-23. 
Glisczinski, D. J. (2007). Transformative Higher Education A Meaningful Degree of Understanding. Journal of Transformative Education, 5(4), 317-328.

Gray, M. (March 6, 2012). Social Media: The Muscle Behind the Trayvon Martin Movement. Time. http://newsfeed.time.com/2012/03/26/social-media-the-musclebehind-the-trayvon-martin-movement/\#ixzz2HaMVtRPh

Graham, P. (1999). Critical systems theory: A political economy of language, thought, and technology. Communication Research, 26(4), 482-507.

Green, M.F. (2002) Internationalizing undergraduate education: Challenges and lessonsof success. In Engberg, D. and Green, M.F. (Ed.) Promising practices: Spotlighting excellence in comprehensive internalization. Washington D.C. :American Council on Education. pp. 7-20

Green, M.F. (2003) The Challenge of Internationalizing Undergraduate Education: Global Learning for All. Global Challenges and U.S. Higher Education Conference. Duke University.

Green, M.F. (2007). Internationalizing community colleges: Barriers and strategies. New directions for community colleges, 138, 15-24.

Green, M.F. (2013). Improving and assessing global learning. New York: NAFSA

Grimstead, C.P. (2008). An exploration of American social justice activists:

Developmental experiences of white undergraduate and post-college millennials who are activists for Africa. Dissertation Abstracts International Section A: Humanities and Social Sciences. 2008

Habib, N., Khan, S., and Sterling, J., (Oct. 10, 2012) Attack on teen blogger consumes Pakistan. $C N N$

Hanson, L. (2010). Global Citizenship, Global Health, and the Internationalization of Curriculum : A Study of Transformative Potential. Journal of Studies in International Education 14(1), 70-88.

Hanvey, R. G. (1976). An attainable global perspective. Denver, CO: Center for Teaching International Relations.

Harari, M. (1992) Internationalization of the curriculum. In Klasek, C.B. (Ed.) Bridges to the Future: Strategies for internationalizing higher education. Carbondale, IL.: Association of International Education. Pp. 52-79.

Harder, A., Lamm, A., Roberts, T.G., Navarro, M., \& Ricketts, J. (2012). Using a reflective Activity to Identify Faculty Beliefs Prior to an International Professional Development Experience. Journal of Agricultural Education, 53,(4), 17-28. DOI: 10.5032/jae.2012.04017 
Harfoush, R. (2009). Yes we did: An inside look at how social media built the Obama brand. New Riders Pub.

Hendershot, K. (2010). Transformative Learning and Global Citizen Identity Development in Undergraduates: A Case Study. Dissertation, Lehigh University

Herbers, M. S. (1998). Perspective transformation in preservice teachers. Unpublished doctoral dissertation, University of Memphis, Tennessee

Hett, E. J. (1993). The development of an instrument to measure global-mindedness. (Doctoral dissertation). Retrieved from ProQuest dissertations \& theses.(Publication No.AAT 9408210).

Heydet-Kirsch, P.E., and Snellgrove, L. (2015). Diversity data report 2014-2015 submitted to the Florida Atlantic University College of Education Executive Committee.

Hicks, D. (2003). Global education: what does it mean? The Challenge of the Dimension in Education-Lecture Series No 1 (2004). London: Institute of Education.

Hoffman-Kipp, P. , Artiles, A.J., and López-Torres, L. (2003) Beyond Reflection: Teacher Learning as Praxis, Theory Into Practice, 42:3, 248-254, DOI: 10.1207/s15430421tip4203_12

Holden, C. (2000). Learning for democracy: From world studies to global citizenship.Theory into practice, 39(2), 74-80.

Hovey, R. and Weinberg, A. (2009). Global Learning and the Making of Citizen Diplomats. In Lewin. R. (Ed.) (2009) Handbook of Practice and Research in Study Abroad : Higher Education and the Quest for Global Citizenship. Florence, KY, USA: Routledge. pp. 33

Hovland, K. (2005a). Shared futures: Global learning and social responsibility.Washington, D.C: AAC\&U.

Hovland, K. (2005b). Shared Futures: Global learning and shared responsibility. Diversity Digest. 8(3) pp. 1, 16-17.

Hovland, K. (2005c). Shared futures? The interconnections of global and U.S. diversity. Diversity Digest. 8(3) pp. 5, 19.

Hovland, K. (2006). Shared futures: Global learning and liberal education. Washington, DC: Association of American Colleges and Universities. 
Howard, P. N. and Parks, M. R. (2012), Social Media and Political Change: Capacity, Constraint, and Consequence. Journal of Communication, 62: 359-362. doi: 10.1111/j.1460-2466.2012.01626.x

Hubball., H., Collins., J. \& Pratt., J. ( 2005). Enhancing Reflective Teaching Practices: Implications for Faculty Development programs. The Canadian Journal of Higher Education. XXXV, (3), pp $57-81$.

Hudzik, J.K. and Stohl, M. (2009). Modelling assessment of the outcomes and impacts of internationalisation. In de Wit, H. (ed.) (2009). Measuring success in the internationalisation of higher education: EAIE Occasional Paper 22. Amsterdam: European Association for International Education (EAIE). 9-22.

Hudzik, J.K. (2011). Comprehensive Internationalization: From concept to action. NAFSA: Washington, D.C.

Jurgenson N. (2012). When Atoms Meet Bits: Social Media, the Mobile Web and Augmented Revolution. Future Internet. 4(1):83-91.

Kean, P.M. (2010). Emerging Adults’ Perceptions of Learning in an Undergraduate Student Organization for Global Social Justice. Dissertation. Walden University.

Kember, D., Kwan., K., \& Ledesma., J. (2001). Conceptions of good teaching and howthey influence the way adults and school leavers are taught, International Journal of Lifelong Education, 20:5, 393-404

King, K.P. (1997). Examining activities that promote perspective transformation among adult learners in higher education (Doctoral dissertation). Available from ProQuest Dissertations and Theses database. (AAT 9724492)

King, K.P. (1998). A guide to perspective transformative and learning activities: TheLearning Activities Survey. Philadelphia: Research for Better Schools.

King, K.P. (2000). The adult ESL experience: Facilitating perspective transformation inthe classroom. Adult Basic Education: An Interdisciplinary Journal for Adult Literacy Education, 10(2) 69-89.

King, K.P. (2002). Educational technology professional development as transformationallearning opportunities. Computers and Education: An International Journal39(3), 283-297.

King, K.P. (2003). Exploring feminist research and pedagogy in the shadow of tragedy:International perspectives construct a response in lifelong learning. Radical Pedagogy, 5(2), 19-26.

King, K. P. (2004). Both sides now: Examining transformative learning and professional development of educators. Innovative Higher Education, 29(2), 155-173. 
King, K.P.. (2009). Evolving Research of transformative learning based on the learning activities survey. Adult education special topics: Theory, research and practice in lifelong learning (pp. 3-94). Charlotte, NC: Information Age.

Kirkwood, T. F. (2001) Our global age require global education: Clarifying definitional ambiguities. The Social Studies 92 (1), 10-15.

Knight, J. (2013). Concepts, rationales, and interpretive frameworks in the internationalization of higher education. In Deardorff, D.K., de Wit, H., Heyl, J.D., and Adams, T. (eds.) (2013). The Sage Handbook of International Higher Education. (pp. 27-43) SAGE Knowledge: New York.

Knight, J. (1999) Internationalisation of higher education. In Quality and internationalisation in higher education. Paris, France: Organization for Economic Co-operation and Development. pp. 13-28.

Knight, J. (2003). Updating the definition of internationalization. International Higher Education, 33, 2-3.

Lakshmi, R. (Jan. 5, 2013). India struggles with social media following rape uproar. The Washington Post. http://www.washingtonpost.com/world/ asia_pacific/india-struggleswith-social -media-following-rape-uproar/2013/01/04/7896933e-559a-11e2-89de 76c1c54b1418_print.html

Lamy, S. (2007). Challenging hegemonic paradigms and practices: Critical thinking and active learning strategies for international relations. PS: Political science and politics, 40(1), 112-116.

Landorf, H. and Doscher, S. P., (2015). Defining Global Learning at Florida international University. Diversity \& Democracy. 18(3), 1-3.

Landorf, H., and Doscher, S. P., (2013). Global Learning for Global Citizenship. In M. Walker and A. Boni. (Eds). Universities and human development: A sustainable imaginary for XXI Century. New York, NY: Routledge Press.

Landorf, H., \& Pineda, F. (2011). Global learning and the human capability approach: Florida International University case study. In M. S. Plakhotnik, S. M. Nielsen, \& D. M. Pane (Eds.), Proceedings of the Tenth Annual College of Education \& GSN Research Conference (pp. 116-122). Miami: Florida International University. http://coeweb.fiu.edu/research_conference/

Landorf, H., Rocco, T. S., \& Nevin, A. (2006). Creating permeable boundaries: Inclusive educators in a global society. In M. S. Plakhotnik \& S. M. Nielsen (Eds.), Proceedings of the Fifth Annual College of Education Research Conference: Urban and International Education Section (pp. 58-63). Miami: Florida International University. http://coeweb.fiu.edu/research_conference/. 
Lawler, P. A., \& King, K. P. (2000). Preparing for effective faculty development: Using adult learning strategies. Malabar, FL: Krieger.

Lenz, B.K. and Warner, S. (2011). Global learning experiences during a domestic community health clinical. Nursing education perspectives. 32 (1), 26-29.

Leskes, A. (2004). Greater Expectations and Learning in the New Globally Engaged Academy. PeerReview: AACU. pp 4-7.

Lister, I. (1995) Educating beyond the Nation International Review of Education. 41, 1/2, Moral Education , 109-118.

Lunn, J. (2008) Global Perspectives in Higher Education: Taking the Agenda Forward inthe United Kingdom. Journal of Studies in International Education, 12(3), 231254.

Mahoney, J. (2004) Revisiting general theory in historical sociology. Social Forces 83 (2). 459-489.

Marquardt, M., \& Reynolds, A. (1994). The global learning organization:Gaining competitive advantage through continuous learning. Burr Ridge, IL: Irwin.

Mazzola, M. C. (2007). Internationalization of graduate and professional programs at the University of Pennsylvania: An opportunity and a challenge. A dissertation inhigher education management

Mclean, M., Cilliers, F. \& Van Wyk, J.M. (2008). Faculty development: Yesterday, today AndTomorrow. Medical teacher 30, (36), 555-584.

Merryfield, M. (1992). Preparing social studies teachers for the twenty-first century: Perspectives on program effectiveness from a study of six exemplary teacher education programs in global education. Theory and Research in Social Education, 20(1), 17-26

Merryfield, M. (1993). Reflective Practice in Global Education: Strategies for Teacher Educators. Theory into Practice, 32(1), 27-32.

Merryfield,M.M. (1997). A framework for teacher education. In Preparing teachers to Teach global perspectives: A handbook for teachers educators, eds. M.M. Merryfield, E. Jarchow, and S. Pickert, 1-24. Thousand Oaks, CA: Corwin Press.

Merkx, G. and de Wit, H. (2013). The History of internationalization in higher education.In Deardorff, D.K., de Wit, H., Heyl, J.D., and Adams, T. (eds.) (2013). The Sage Handbook of International Higher Education. (pp. 43-61) SAGE Knowledge: New York. 
Mezirow, J. (1978). Education for perspective transformation; women's re-entryprograms in community colleges. New York: Teacher’s College, Columbia University.

Mezirow, J. (1994). Understanding transformation theory. Adult Education Quarterly, 44(4), 222-232.

Mezirow, J. (2000). Learning to think like an adult. In J. Mezirow, \& X. Associates (Eds.), Learning as transformation: critical perspectives on a theory in progress (pp. 3-34). San Francisco: Jossey-Bass.

Mezirow, J., \& Associates. (1990). Fostering critical reflection in adulthood; a guide to transformative and emancipatory learning. San Francisco: Jossey-Bass.

Mezirow, J., \& Associates. (2000). Learning as transformation: critical perspectives on a theory in progress. San Francisco: Jossey-Bass.

Mezirow, J. (2000). Learning as transformation: Critical perspectives on a theory in progress. San Francisco: Jossey Bass.

Musil, C. M. (2006). Assessing global learning: Matching good intentions with good practice. Washington, DC: Association of American Colleges and Universities.

Nagda, B. A., Gurin, P., \& Lopez, G. E. (2003) Transformative pedagogy for democracy and social justice. Race Ethnicity \& Education, 6(2), 165-191.

Navarro, M. (2004). Analysis of factors affecting participation of faculty and choice of strategies for the internationalization of the undergraduate agricultural curriculum: The case in two land grant universities (Unpublished doctoral dissertation). Texas A\&M University, College Station, TX.

Nelson, W. (1983). 'Faculty who stay: Renewing our most important resource', in

Baldwin,R. and Blackburn, R. (eds.), College Faculty: Versatile Human Resources in a Period of Constraint. San Francisco, CA: Jossey-Bass Publishers, pp. 67-83.

OECD (1999). Quality and internationalisation in higher education. Paris, France: Organization for Economic Co-operation and Development.

OECD (1996). Internationalising the curriculum in higher education. Paris, France: Organization for Economic Cooperation and Development .

Olson, C. L., Green, M. F., \& Hill, B. A. (2006). A handbook for advancingcomprehensive internationalization: What institutions can do and what students should learn. Washington, DC: American Council on Education.

Olson, C.L. and Kroeger, K.R. (2001). Global Competency and Intercultural Sensitivity.Journal of Studies in International Education, 5 (2), 116-137 
Perry, S.E. and Mander, R. (2005). A global frame of reference: Learning from everyone, everywhere. Nursing education perspectives. 26, 3. pp. 148- 151.

Peters-Davis, N.D. (2005). Connecting the Global and the Local: The Experience of Arcadia University. Diversity Digest. 8(3) pp. 6, 23.

Pinar, W.F. (2007) Crisis, reconceptualization, internationalization: U.S. Curriculum theory since 1950. Shanghai: East China Normal University.

Powell, S., Tindal, I., and Millwood, R. (2008) Personalized learning and the Ultraversity experience. Interactive Learning Environments 16(1), 63-81

Pratt, D. D. (1992). Conceptions of teaching. Adult Education Quarterly, 42(4), 203-20.

Pratt, D. D. (2002).Good teaching: one size fits all? In An Up-date on Teaching Theory, JovitaRoss-Gordon (Ed.), San Francisco: Jossey-Bass.

Regent university (2009). Developing Globally Competent Christian Leaders Quality Enhancement Plan Regent University January 20, 2009

Riegle, R. (1987). 'Conceptions of faculty development', Educational Theory 37, 53-59.

Rehm, M. (2009). Unified in Learning--Separated by Space: Case Study of a Global Learning Programme. Industry and Higher Education, 23(4), 331-341.

Riel, M. (1995) Cross-classroom collaboration in global learning circles. In Star, S. L (Ed.) (1995). The culture of computing. Oxford, UK. Blackwell publishers. Pp. 219-239.

Rimmington, G. (2003). An Introduction to Global Learning. In C. Crawford et al. (Eds.), Proceedings of Society for Information Technology \& Teacher Education International Conference 2003 (pp. 1536-1539). Chesapeake, VA: AACE.

Rimmington, G., Gibson, K., Gibson, I. \& Alagic, M. (2004). The cage model of global learning. In R. Ferdig et al. (Eds.), Proceedings of Society for Information Technology \& Teacher Education International Conference 2004 (pp. 30273032). Chesapeake, VA: AACE.Retrieved from http://www.editlib.org/p/13312.

Romney, A., Batchelder, W., and Weller, S. (1986). Culture as consensus: A theory of culture and informant accuracy. American Anthropologist. 88, 313-332.

Russell, D. R. (1997) Rethinking genre in school and society: An activity theory analysis.Written Communication, 14(4), 504-554. 
Ryan, G.W. and Bernard, H.R. (2003). Techniques to Identify Themes. Field Methods, 15(1), 85-109St. Edward's University, (2007). Developing Global Understanding in Undergraduate Students A Quality Enhancement Plan Submitted by St. Edward's University To the Southern Association of Colleges and Schools Commission on Colleges Feb. 8, 2007

Schneider, C.G. (2007). Civic Learning in a Diverse Democracy: Education for Shared Futures. Diversity \& democracy: Civic learning for Shared Futures. 10(3), 1-3.

Schnick, C., and Petrequin, P., (2007). Deconstructing the American Dream through Global Learning. Diversity \& democracy: Civic learning for Shared Futures. Vol. 10, 3. Pp 10-11.

Schuerholz-Lehr, S. (2004) Teaching for Global Literacy in Higher Education: How Prepared Are the Educators? Journal of Studies in International Education. 11;(2) $180-204$.

Schenck, C. (2005). Hybrid Student Identities: A Resource for Global Learning. Diversity Digest. 8(3) pp. 4, 15

Schmidt, L. (2005) Looking Within to See the World Diversity Digest. 8(3), 8-9, 20

Shultz, J., Skilton-Sylvester, E., and Shultz, N.P. (2007). Exploring Global Connections: Dismantling the International/Multicultural Divide. Diversity \& democracy: Civic learning for Shared Futures. Vol. 10, 3. Pp 4-6.

Shultz, L. \& Jorgenson, S. (2008). Global citizenship education in post secondary institutions: A review of the literature. Accessed January 5th, 2008, fromhttp://www.uofaweb.ualberta.ca/uai_globaleducation/pdfs/GCE_lit_review.p df

Shultz, L. (2007). Educating for global Citizenship: Conflicting Agendas and Understandings. Alberta Journal of Educational Research, 53(3). 248-258.

Schon, D. (1983). The reflective practitioner." How professionals think in action. New York: Basic Books.

Schuster, J. (1990). 'The need for fresh approaches to faculty renewal', in Schuster, J.,Wheeler, D. and Associates (eds.), Enhancing Faculty Careers: Strategies for Development and Renewal. San Francisco, CA: Jossey-Bass Publishers, pp. 319.Sikes, W. and Barrett, L. (1976). Case Studies on Faculty Development. Washington, D.C.: Council for the Advancement of Small Colleges.

Silveira, C. (2007). Art and Social Action in Cambodia: Transforming Students into World Citizens. Diversity \& democracy: Civic learning for Shared Futures. .10,(3). 7-9. 
Sleeter, C.E. (1996). Multicultural Education as Social Activism (Albany, NY, SUNYPress).

Smith, D. G., Esau, T., and Garcia, H.A. (2012). Where are they? A multilens examination of the distribution of full-time faculy by institutional type, race/ethnicity, gender, and citizenship. New Directions for institutional research, 155, 5-26.

Smith, J. M., and Kruse, J. (2009). "Making Connections” at The University of North Carolina: Moving Toward a Global Curriculum at a Flagship Research University. The Journal of General Education, 58(2), 106-120.

Sperandio, J., Grudzinski-Hall, M., and Stewart-Gambino, H. (2010). Developing an Undergraduate Global Citizenship Program: Challenges of Definition and Assessment. International Journal of Teaching and Learning in Higher Education. 22, (1), 12-22.

Spiegelberg, H. (1982). The phenomenological movement (3rd ed.). The Hague, Netherlands: Martinus Nijhoff.

Spinelli, G. (2009). Measuring the success of internationalisation: the case for joint and double degrees. In de Wit, H. (ed.) (2009). Measuring success in the internationalisation of higher education: EAIE Occasional Paper 22. Amsterdam: European Association for International Education (EAIE). 49-56.

Stamats, Inc. (2008). Florida International University Brand Research Report. Cedar Rapids: Stamats.

Sullivan, L. (1983). 'Faculty development: A movement on the brink', The College Board Review 127, 21 and 29-31.

Takacs, S. (2008) Making Globalization Ordinary: Teaching Globalization in the American Studies Classroom American Studies, 49:3/4 : 221-254.

Taylor, E. W. (2007). An update of transformative learning theory: A critical review ofthe empirical research (1999-2005). International Journal of Lifelong Education, 26, 173-191.

Temple-Thurston, B. (2005). Student Civic Engagement at Home and Abroad. Diversity Digest. 8(3) pp. 8-9.

Thornton, S. J. (1988). Curriculum consonance in United States history classrooms. Journal of Curriculum and Supervision, 3(4), 308-320.

Tolbert, A.S., McLean, G.N., and Myers, R.C. (2002). Creating the global learning organization (GLO). The International Journal of InterculturalRelations. 
Tye, K. (ed.) (1991). Global Education: From Thought to Action (Alexandria, Va.: Association for Supervision and Curriculum Development, 1991), p. 5.

Tye, K. (1999). Global Education: A Worldwide Movement (Orange, Calif.: Interdependence Press, 1999).

Volet, S. (2004). Challenges of internationalisation: Enhancing intercultural competence and skills for critical reflection on the situated and non-neutral nature of knowledge. In P. Zeegers \& K. Dellar-Evans (Eds.), Language \& academic skills in higher education (Vol. 6, pp. 1-10). Adelaide, Australia: Flinders University.

Wallerstein, I. (1991) Unthinking social science: the limits of nineteenth-century paradigms. Cambridge: Polity Press.

Wallerstein, I, chair. (1996) Open the social sciences: Report of the Gulbenstein Commission on the restructuring of the social sciences. Stanford, Ca: Stanford University press,

Weber, N. (2008, Oct). Global Education in Canada, the United Kingdom and Ireland:Institutional Practices and Programming at the Post-Secondary Level. Paper presented at the Global Citizenship Education and Post Secondary Institutions:Policies, Practices and Possibilities Conference, Edmonton, AB.

White, J.L. (2005). Introducing undergraduates students to global health challenges through web based learning. Web Learning. Nursing education perspectives. 26(3), 157-162.

Woolf, M. (2009). Measuring success in education abroad: Who are we trying to impress? In de Wit, H. (ed.) (2009). Measuring success in the internationalisation of higher education: EAIE Occasional Paper 22. Amsterdam: European Association for International Education (EAIE). 57-64.

Wiggins, G., \& McTighe, J. (2005). Understanding by design. Alexandria, VA: Association for Supervision and Curriculum Development.

Zemach-Bersin, T. (2007). Global citizenship and study abroad: It's all about U.S. Critical Literacy: Theories and Practices, 1(2). 16-28.

Zinn, L. F. (1997). Supports and barriers to teacher leadership: Reports of teacher leaders. Unpublished doctoral dissertation, University of Northern Colorado, Greeley, CO.

Zuber, B. (2008, Oct). Good works in important places: Risk-adverse globalcitizenship and its enabling institutions. Paper presented at the Global Citizenship Education and Post Secondary Institutions: Policies, Practices and Possibilities Conference, Edmonton, AB. 


\section{APPENDICES}




\section{APPENDIX A}

Global Learning Survey 


\section{GLOBAL LEARNING SURVEY}

1 Consent to participate

- $\quad$ Online the respondent must read a consent form and acknowledge consent by clicking on a consent button or the respondent will not be allowed to view the rest of the survey.

2.Did the global learning workshops cause you to reconsider the way you teach? Yes or no.

- If this question is answered in the negative, ithe survey skips to question \#4.

3.Please explain how the global learning workshops caused you to reconsider the way you teach.

- $\quad$ This question was not displayed to the respondent if the respondent answered no to the previous question.

4.Did the global learning workshops cause you to reconsider the content of your courses? Yes or no. 
- If this question is answered in the negative, the survey skips to question

$\# 6$.

5.Please explain how the global learning workshops caused you to reconsider the content of your courses.

- This question was not displayed to the respondent if the respondent answered no to the previous question.

6.Did the global learning workshops cause you to reconsider your relationship with your students? Yes or no.

- If this question is answered in the negative, the survey skips to question

\#8.

7.Please explain how the global learning workshops caused you to reconsider your relationship with your students. 
- $\quad$ This question was not displayed to the respondent if the respondent answered no to the previous question.

8.Did the global learning workshops cause you to reconsider your views on learning? Yes or no.

- If this question is answered in the negative, the survey skips to question $\# 10$.

9.Please explain how the global learning workshops caused you to reconsider your views on learning.

- $\quad$ This question was not displayed to the respondent if the respondent answered no to the previous question.

10.Did you learn something due to the global learning workshops that made you feel uneasy? Yes or no. 
- If this question is answered in the negative, the survey skips to question

$\# 12$.

11.Please explain what you learned due to the global learning workshops that made you feel uneasy.

- $\quad$ This question was not displayed to the respondent if the respondent answered no to the previous question.

12.Due to the global learning workshops, did you experience something that contradicted a previously held belief? Yes or no.

- If this question is answered in the negative, the survey skips to question

$\# 14$.

13.Please describe the experience that contradicted a previously held belief. 
- $\quad$ This question was not displayed to the respondent if the respondent answered no to the previous question.

14.Did you have an epiphany due to the global learning workshops? Yes or no.

- If this question is answered in the negative, the survey skips to question \#16.

15.Please describe this epiphany due to the global learning workshops.

- $\quad$ This question was not displayed to the respondent if the respondent answered no to the previous question.

16.Did you recognize a shared process of transformation shared by you and others due to the global learning workshops? Yes or no. 
- If this question is answered in the negative, the survey skips to question $\# 18$.

17.Please describe this shared process of transformation shared by you and others due to the global learning workshops.

- This question was not displayed to the respondent if the respondent answered no to the previous question.

18.Did you develop new relationships due to the global learning workshops? Yes or no.

- If this question is answered in the negative, the survey skips to question \#20.

19.Please describe the nature of these new relationships due to the global learning workshops. 
- $\quad$ This question was not displayed to the respondent if the respondent answered no to the previous question.

20.Was there a change in your self confidence due to the global learning workshops? Yes or no.

- If this question is answered in the negative, the survey skips to question \# 22.

21.Please describe the change in your self confidence due to the global learning workshops.

- $\quad$ This question was not displayed to the respondent if the respondent answered no to the previous question.

22.How many global learning courses do you teach? 
- $\quad$ Answers can vary.

23.How often do you teach a global learning course?

- $\quad$ Answers can vary.

24.How long have you been teaching a global learning course?

- $\quad$ Answers can vary.

25.How would you describe your ethnicity?

- $\quad$ Answers can vary.

26.What is your race? Please select. 
- The respondent has the following options for an answer.

White (Non-Hispanic)

Hispanic

Asian or Pacific Islander

Other (Please describe)

27.In which FIU school or college do you teach?

- $\quad$ The respondent has the following options for an answer.

College of Architecture and Arts

College of Arts and Sciences

College of Business

College of Education

College of Engineering and Computing

Honors College

College of Law

Herbert Wertheim College of Medicine

Nicole Wertheim College of Nursing and Health Sciences

Chaplin School of Hospitality and Tourism Management

School of Journalism and Mass Communications 
Other: (Please describe)

28.I am interested in conducting follow up semi-structured interviews with some of the faculty members who took this survey about their experiences as adult learners and educators. The interviews will be conducted at a time and place of your convenience and will last about thirty minutes. Your information will be kept confidential. Your participation would be of great service to the university and towards research scholarship. Are you willing to participate in a follow up interview?

- The respondent has the option of yes or no. The survey ends if the respondent answers in the negative.

29.If "Yes," please give your name and email and/or other contact information so that I can schedule a possible interview.

- $\quad$ Answers can vary. The survey ends once this question is answered. The respondent can not return to the survey. 
APPENDIX B

Semi-structured interview guide 
Exploring how Transformational Experiences of Faculty in Global Learning Workshops inform Practice

This interview is part of research that included the survey you completed on global learning and transformational learning. This dissertation explores the experiences of faculty members as adult learners and adult educators. In this interview, you will be asked to reflect on your experiences in the global learning professional development workshop. The interview will take approximately a half an hour. Your responses will be anonymous. With your permission, the interview will be audio recorded and later transcribed into a Microsoft Word document. At a later date, the researcher will email you preliminary findings as a form of member checking. You will be invited to give feedback on these findings.

1. Tell me about your experiences in the global learning workshop.

2. Describe an aspect of the global learning workshop that you found engaging.

3. What was the most engaging aspect about the global learning workshop?

4. Describe an experience or dilemma you had to struggle with because of the global learning workshop.

5. Describe an important idea, role, relationship, or action you had to rethink because of the workshop. 
6. Describe how your experiences in the global learning workshop made you question, challenge, or view in a more complex way a belief you took for granted.

7. Describe an instance when the global learning workshop made you think about teaching differently.

8. Did the global learning workshop inspire you to revise your global learning course? If so, how?

9. Did you make changes to other courses you teach due to the workshop? If so, please tell me about these changes.

10. How do you think your experience with the global learning workshop has affected student learning?

11. Have you shared your experience in the workshop with anyone? If so, please describe what you shared and with whom.

12. Please tell me anything that you would like to add.

Thank you. 


\section{APPENDIX C}

GL Foundations Course Approval Checklist 


\section{GLOBAL LEARNING FOUNDATIONS COURSE APPROVAL CHECKLIST}

1. Does the syllabus state the global learning course learning outcomes?

The course outcomes must address both the global learning student learning outcomes and the course content. The outcomes must be measurable and address higher order thinking skills (analysis, synthesis, and evaluation).

2. Does the course include a comprehensive assessment plan for the global learning course learning outcomes?

The assessment plan for the global learning course outcomes must include a description of appropriate assessment artifacts, evaluation processes, minimum criteria for success, and sampling methods.

3. Does the course address the global learning course outcomes through appropriate interdisciplinary content and readings?

The course must address essential questions associated with global, local, international, and/or intercultural issues and/or problems. Students must engage in interdisciplinary, multi-perspective analysis of issues and/or problems.

Does the course address the global learning course outcomes through active learning strategies?

The course must utilize active learning strategies (e.g. Team-Based Learning, case method of instruction, discussion, debate)

5. Does the course address the global learning course outcomes through an integrated co-curricular activity?

The course must include description of at least one co-curricular activity that addresses the global learning student learning outcomes. 
APPENDIX D

Discipline-Specific GL Course Approval Checklist, 


\section{DISCIPLINE-SPECIFIC GLOBAL LEARNING COURSE APPROVAL CHECKLIST}

1. Does the course include global learning course outcomes?

The global learning course outcomes must address both the global learning student learning outcomes and the course content. The outcomes must be measurable and address higher order thinking skills (analysis, synthesis, and evaluation).

2. Does the course include a comprehensive assessment plan for the global learning course outcomes?

The assessment plan for the global learning course outcomes must include a description of appropriate assessment artifacts, evaluation processes, minimum criteria for success, and sampling methods.

3. Does the course address the global learning course outcomes through appropriate content and readings?

The course must address essential questions associated with global, local, International, or intercultural issues and/or problems. Students must engage in multi-perspective analysis of issues and/or problems.

4. Does the course address the global learning course outcomes through active learning strategies?

The course must utilize active learning strategies (e.g. Team-Based Learning, case method of instruction, discussion, debate) 


\section{APPENDIX E}

Model GL Syllabus (Annotated) 


\section{Artistic Expression in a Global Society \\ A Global Learning ${ }^{1}$ Foundations Course}

\section{FIU ceabaning}

IDS 3336-U01, Course \# 10412, 3 Credits

Course Website Login: http://ecampus.fiu.edu

Time: Wednesday 2 - 4:45 PM, RDB 1100

\begin{tabular}{|c|c|c|c|}
\hline $\begin{array}{c}\text { Steven Schoen, Ph.D } \\
\text { Communication Arts }\end{array}$ & $\begin{array}{c}\text { M. Stephanie Chancy } \\
\text { Art History }\end{array}$ & $\begin{array}{c}\text { Matthew Tobin } \\
\text { Teaching Assistant - } \\
\text { Music }\end{array}$ & $\begin{array}{c}\text { AdrienneRose Gionta } \\
\text { Teaching Assistant - } \\
\text { Art }\end{array}$ \\
\hline swschoen@fiu.edu & chancyms@fiu.edu & thetobinator@gmail.com & agionta@fiu.edu \\
\hline Phone: 305-348-0552 & Phone: 305-348-2897 & & \\
\hline Office: VH 205 & Office: VH 216 & & Office Hours: \\
\hline $\begin{array}{c}\text { Office Hours: } \\
\text { T/W 10AM - 1PM } \\
\text { \& by appointment }\end{array}$ & $\begin{array}{c}\text { T/R 11AM - 12PM } \\
\text { \& by appointment }\end{array}$ & $\begin{array}{c}\text { Office Hours: } \\
\text { By Appointment }\end{array}$ & $\begin{array}{c}\text { Office Hours: } \\
\text { By Appointment }\end{array}$ \\
\hline
\end{tabular}

Spring 2013

NOTE: The best way to reach us is by email, NOT by telephone!

University Drop Date: March 18, 2013 Last day to drop a course with a DR grade

COURSE DESCRIPTION

A

rtistic expression varies from culture to culture, and what constitutes artistic expression depends on the norms, attitudes, values, beliefs, and social practices of a given culture. All cultures - past and present - enlist language, music, and art to

communicate their visions, hopes, and dreams and to respond and react to events in their daily lives and societies. While the appreciation of the various modes of artistic expression present in diverse cultures is its own reward, multi-cultural awareness also facilitates the unprecedented crossnational interaction and creative collaboration recently made possible through rapid technological innovations such as iTunes, YouTube, Internet 2, etc. The motivation for such a course flows from the following conditions:

- Cultures cannot endure without artistic expression. Societies will continue to grow, prosper and adapt to an ever-changing world dynamic but at what cost? As the world becomes more interconnected, attitudes, values, beliefs, norms, and social practices of any given culture are at risk of being marginalized or even lost.

Boilerplate text noting that this is a Global Learning course.

1This is a Global Learning Foundations course that counts towards your Global Learning graduation requirement. 
Creative Engagement Project (10 points)

The purpose of this assignment is for you to experience art as a way to engage people around issues of global concern. You will do this assignment in groups of 4-7 people that you can choose yourselves (you are also welcome to ask permission do this activity on your own). You will plan and execute an art project that uses art to engage people in a public way in order to make a positive difference about an issue of global concern.

Mid-Term Exam (15 points)

This exam will be taken in class. Please bring a \#2 Pencil.

Final Exam (20 points)

This exam is cumulative and will be taken in class. Please bring a \#2 Pencil.

\section{Policy for Assigning an “I” INCOMPLETE GRADE}

An incomplete grade is a temporary symbol given for work not completed because of serious interruption not caused by the student's own negligence. Please see FIU's Policy for Assigning an Incomplete "I" Grade.

\section{CenTER FOR ACADEMic Success}

The Center for Academic Success is available on both campuses to support you. They provide personalized attention tailored to your needs in a user-friendly environment that includes online support. You can get help writing a paper, reading more efficiently and increasing textbook comprehension, or even creating an individualized learning plan. The center is located in Green Library 120, next to Starbucks (305-348-2441) at MMC and in ACI 160 (305-919-5927) at BBC. Find them online at http://learningcenter.fiu.edu.

\section{ACADEMic Misconduct}

Florida International University is a community dedicated to generating and imparting knowledge through excellent teaching and research, the rigorous and respectful exchange of ideas, and community service. All students should respect the right of others to have an equitable opportunity to learn and honestly to demonstrate the quality of their learning. Therefore, all students are expected to adhere to a standard of academic conduct, which demonstrates respect for themselves, their fellow students, and the educational mission of the University. All students are deemed by the University to understand that if they are found responsible for academic misconduct, they will be subject to the Academic Misconduct procedures and sanctions, as outlined in the Student Handbook. This Code of Academic Integrity was adopted by the Student Government Association on November 28, 2001 and reflects the values articulated in the Student Code of Standards.

All students are deemed by the university to understand that if they are found responsible for academic misconduct, they will be subject to the Code of Academic Integrity's procedures and sanctions, as outlined in the FIU Student Handbook. Students have the right to due process in all disciplinary situations. For additional information concerning student rights and responsibilities, please contact FIU's Office of Student Conduct and Conflict Resolution.

Academic misconduct will not be tolerated in this class. Ignorance of the law is no excuse. Violations of academic integrity will be punished. These violations involve the use of any method or technique enabling you to misrepresent the quality or integrity of any of your university related work.

Students committing academic dishonesty (cheating on tests, plagiarism, etc.) may be reported to university officials and in all cases will be dealt with severely. This website offers the sanctions instituted at Rutgers University for every type of infraction:

http://www.camden.rutgers.edu/RUCAM/info/Academic-Integrity-Policy.html. Our approach will be similar. If you have not already done so, please read through the Standards of Student Conduct in your FIU Student Handbook. The Standards address three major areas of moral integrity: Academic Honesty, Respect for the Law, and Respect for People.

CODE OF STUDENT CONDUCT 
Florida International University is a learning community following a tradition more than 1,000 years old that is dedicated to generating and imparting knowledge through excellent teaching and research, the rigorous and respectful exchange of ideas, and community service. Students are expected to respect this tradition of academic inquiry, the University's rules of conduct, its mission, and the opinions and differences of all members of the FIU community. Civility is essential. The FIU Student Handbook outlines the Student Code of Conduct regarding students with disruptive behavior: www.fiu.edu/ sccr/docs/disruptive_brochure.doc

\section{Disability Clause}

Students with disabilities, as defined by law, have the right to receive needed accommodations if their disabilities make it difficult to perform academic tasks in the usual way or in the allotted time frame. In order to receive accommodation, however, students with must register with Disability Resource Center:

University Park Campus, GC 190

Voice: (305) 348-3532

TTY: (305) 348-3852

Fax: (305) 348-3850

Email: drcupgl@fiu.edu 
The following is the class schedule with the appropriate reading assignments. To get the most out of our sessions together, it is imperative for you to complete the reading or video assignment before we meet. Items to be read or viewed are listed in the Class Schedule on the day they will be discussed, in other words, the time by which your preparation must have been completed. Activities below that involve an assignment or assessment are indicated in red.

speakers, assignments, and assessments.

Schedule shows how global learning is interwoven

Week 1

Wednesday

Jan 9

Course Introduction \& Syllabus

Essential Questions, In-class Assessment Assignment \& Discussion: “What is Expression?"

Week 2

Creativity

Wednesday

Jan 16

Read/View before class: Creativity: Spivey, Nigel "The Birth of the Imagination' Chapter excerpt from How Art Made the World

Read before class: Csikszentmihalyi, Mihaly. Creativity: Flow and the Psychology of Discovery and Invention. New York: Harper Collins, 1996, pages 343-372

View before class: Ken Robinson Says that Schools Kill Creativity

http://www.ted.com/talks/ken_robinson_says_schools_kill_creativity.html

\section{Week 3}

General Concepts, Theory, \& Methodology: An Introduction To Communication \& Art Around The

Wednesday Globe

Jan 23

Read/ View before class: Methodology: Sayre, Henry M. "Using Visual Information: What to look for and how to Describe what you see" Chapter excerpt from Writing About Art

Read before class: Adichie, Chimamanda Ngozi. "You in America." http://www.allstory.com/extra/issue38/adichie.html (short story)

Read before class: World Bank brief on Intercultural Communication http://wwwwds.worldbank.org/external/default/WDSContentServer/WDSP/IB/2010/01/11/000333037201 00111001827/Rendered/PDF/526180BRI0Inte10Box345574B01PUBLIC1.pdf

Post to Course Discussion Board on Blackboard under "Global 1"

Week 4

Wednesday

Creativity As Social Engagement: Introduce Creative Activity Project, View Examples Of Art Used As

Jan 30 Social Engagement, Guest Speaker - Artist Xavier Cortada

Read/View before class: [TBA]

Post to Course Discussion Board on Blackboard under "Creativity" 
Week 5

Wednesday

Feb 6
Art Around The Globe: How It Is Developed And Consumed Similarly And Differently From Culture To Culture

Pre-Discussion quiz on art materials for this class session.

Read/View before class: Art Around the Glove: Spivey, Nigel "More Human than Human" Chapter excerpt from How Art Made the World

Post to Course Discussion Board on Blackboard under "Art"

\section{Week 6}

Wednesday

Feb 13

Thinking About Culture Through The Lens of Language: Lecture, Discussion, And Activity

Pre-discussion quiz on Communication materials for this class session.

Read before class: Deutscher, Guy. "Does Your Language Shape How You Think?" The New York

Times. Aug 8, 2010. http://www.nytimes.com/2010/08/29/magazine/29language-

t.html?pagewanted=all

Read before class: Lakoff, George. 2004. Don't Think of an Elephant. Chelsea Green Publishing. pp. $\mathrm{xv}$-xvi, 3-14. (pdf on Blackboard).

Read before class: Excerpts from Roland Barthes (pdf on Blackboard).

Post to Course Discussion Board on Blackboard under "Communication"

Week 7

Wednesday

Feb 20

Midterm Exam // Guest Speaker - Klaudio Rodriguez, Curator at the Frost Art Museum

Creative Engagement / Group Project Proposal Due

Week 8

Wednesday

Feb 27

Aesthetics: One Person's Trash Is Another's Person's Treasure, Or Is It?

Read/View: [Art Reading?]

Read: Chinua Achebe. An Image of Africa. From The Critical Tradition: Classic Texts and

Contemporary Trend,. 3rd Ed. D.H. Richter, Ed. (pdf on Blackboard)

(This is a very long article and you do NOT have to read it all carefully. Please just focus on Achebe's

argument about what makes something "good art" vs "bad art.")

Post to Course Discussion Board on Blackboard under "Aesthetics"

Week 9

Wednesday

Mar 6

\section{Approaches To Religious Expression}

Read: Scott Aran. 2012. God and the Ivory Tower: What we don't understand about religion just might kill us. Foreign Policy.

http://www.foreignpolicy.com/articles/2012/08/06/god_and_the_ivory_tower

Religion Site Visit Assignment (Submit Online AND Bring to Class)
Week 10

Wednesday

Mar 13
SPRING BREAK - NO CLASS 
Week 11

Wednesday

Mar 20

Wednesday

Mar 27

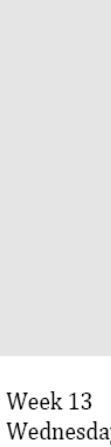

Apr 3

\section{Approaches To Political Artistic Expression}

Read/View: [Art]

Read Excerpt: Foucault on the Panopticon and Governmentality (pdf on Blackboard)

Read: Colin McSwuggen, Design plays a central role in cultural reproduction. This isn't necessarily a good thing, for anyone: http://jacobinmag.com/2012/08/designing-culture/

Post to Course Discussion Board on Blackboard under "Political"

Censorship (viewing of the film Degenerate Art followed by group discussion of the film and readings below)

Read/View: [Art Reading TBA]

Read: Frank Thadeusz, 2010. No Copyright Law: The Real Reason for Germany's Industrial Expansion? Spiegel. http://www.spiegel.de/international/zeitgeist/no-copyright-law-the-realreason-for-germany-s-industrial-expansion-a-710976.html. Focus on the arguments about copyright law and the implications of this for promoting free expression.

ACLU Statement on Artistic Freedom

http://www.aclufl.org/take_action/download_resources/info_papers/14.cfm

Post to Course Discussion Board on Blackboard under "Censorship"

\section{Global Perspectives On Expression}

Pre-discussion quiz on the reading by Kwame Appiah

Read: "The Case for Contamination," New York Times, January 1, 2006. Online at http://www.nytimes.com/2006/01/01/magazine/01 cosmopolitan.html

Class Discussion on Global Learning Common Reading: Appiah, K.A., "The Case for Contamination" and viewing of the film Wasteland.

Post to Course Discussion Board on Blackboard under "Global 2"

Week 14

Wednesday

Apr 10

Matt Tobin - Guest Lecture

Creative Engagement Project presentations

Week 15

Wednesday Creative Engagement Project presentations

April 17

Week 16

Wednesday

Apr 24

Final Exam

Second Essential Questions In-class Assessment Assignment: "What is Expression?"

Time \& Location: TBA 


\section{APPENDIX F}

Model GL Course Outcome Assessment Matrix (Annotated) 


\section{FIU

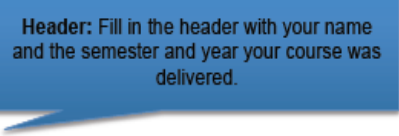

Course Outcome Assessment for Global Learning Courses Faculty Name: XXXXXX XXXXXXXX

Course: ASN 3410, Introduction to East Asia

Academic Unit: Asian Studies Program

Degree Program: BA Asian Studies

Semester Assessed: Spring 2013

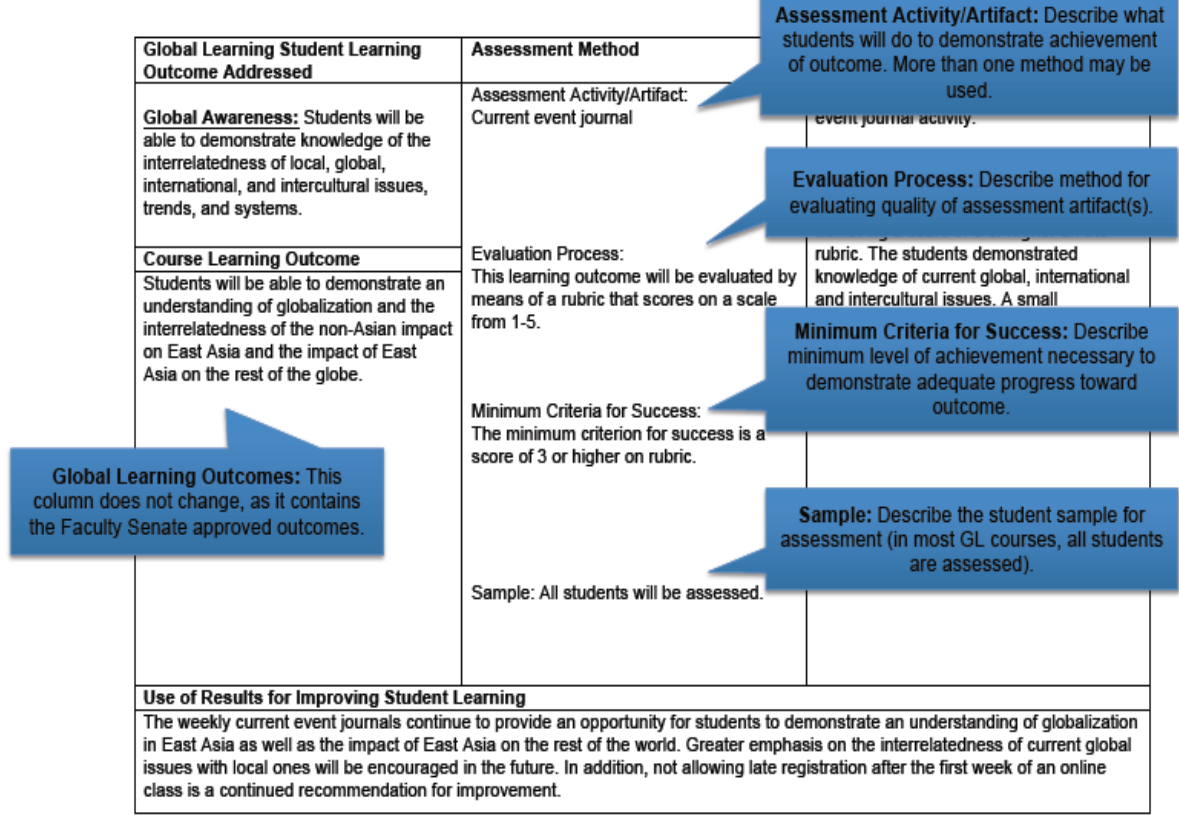




\section{APPENDIX G}

FIU GL Rubrics (Global Awareness and Global Perspective). 
Global Learning Rubrics

\begin{tabular}{|c|c|c|c|c|}
\hline 0 & $\begin{array}{l}\text { (Knowledge/Comprehension) } \\
1\end{array}$ & (Application) & $\begin{array}{l}\text { (Analysis) } \\
3\end{array}$ & $\begin{array}{l}\text { (Synthesis/Evaluation) } \\
4\end{array}$ \\
\hline $\begin{array}{l}\text { Does not demonstrate } \\
\text { knowledge of issues } \\
\text { influencing the problem } \\
\text { Student accomplishes the } \\
\text { following: } \\
\text { fails to cite issues } \\
\text { influencing the problem } \\
\text { fails to depict } \\
\text { interrelatedness of issues } \\
\text { influencing the problem }\end{array}$ & $\begin{array}{l}\text { - Demonstrates incorrect or } \\
\text { incomplete knowledge of } \\
\text { issues influencing the } \\
\text { problem } \\
\text { Student accomplishes the } \\
\text { following: } \\
\checkmark \text { presents incorrect or } \\
\text { incomplete depiction of issues } \\
\text { influencing the problem } \\
\checkmark \text { presents incorrect or } \\
\text { incomplete depiction of the } \\
\text { interrelatedness of issues } \\
\text { influencing the problem }\end{array}$ & $\begin{array}{l}\text { - Demonstrates, with } \\
\text { generalizations, knowledge } \\
\text { of issues influencing the } \\
\text { problem } \\
\text { Student accomplishes the } \\
\text { following: } \\
\text { limited numberally describes } \\
\text { influencing the problem } \\
\quad \text { implies or generally } \\
\text { describes the interrelatedness } \\
\text { of issues influencing the } \\
\text { problem }\end{array}$ & $\begin{array}{l}\text { - Assembles an analysis of } \\
\text { the problem based on the } \\
\text { interrelatedness of } \\
\text { influencing issues } \\
\text { Student accomplishes the } \\
\text { following: } \\
\text { issues influencing the problem } \\
\text { evidence of or references to } \\
\text { interrelated issues influencing } \\
\text { the problem }\end{array}$ & $\begin{array}{l}\text { - Synthesizes and/or } \\
\text { evaluates differing } \\
\text { interpretations of the } \\
\text { problem based on the } \\
\text { interrelatedness of } \\
\text { influencing issues } \\
\text { Student accomplishes } \\
\text { previous and one or more of } \\
\text { the following: } \\
\text { one interpresetation of the } \\
\text { problem } \\
\text { the problem(s) on the } \\
\text { interrelatedness of } \\
\text { influencing issues }\end{array}$ \\
\hline
\end{tabular}

\begin{tabular}{|c|c|c|c|c|}
\hline \multicolumn{5}{|c|}{ Global Perspective: Ability to develop a multi-perspective analysis of local, global, international, and intercultural problems. } \\
\hline 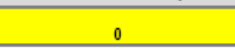 & $\begin{array}{c}\text { (Knowledge/Comprehension) } \\
1\end{array}$ & $\begin{array}{c}\text { (Application) } \\
2\end{array}$ & $\begin{array}{c}\text { (Analysis) } \\
3\end{array}$ & $\begin{array}{c}\text { (Svnthesis/Evaluation) } \\
4\end{array}$ \\
\hline $\begin{array}{l}\text { - Does not recognize or } \\
\text { does not acknowledge } \\
\text { the legitimacy of } \\
\text { differences in } \\
\text { perspectives pertaining } \\
\text { to the problem } \\
\text { Student accomplishes the } \\
\text { following: } \\
\text { perspective and does not } \\
\text { recognize other perspectives } \\
\text { pertaining to the problem }\end{array}$ & $\begin{array}{l}\text { - Identifies a limited number } \\
\text { of perspectives pertaining } \\
\text { to the problem } \\
\text { Student accomplishes the } \\
\text { following: } \\
\text { number of perspectives } \\
\text { pertaining to the problem } \\
\text { identifies only those } \\
\text { perspectives explicitly stated in } \\
\text { article }\end{array}$ & $\begin{array}{l}\text { - Identifies multiple } \\
\text { perspectives pertaining to } \\
\text { the problem however, does } \\
\text { not analyze the influences } \\
\text { on differing perspectives } \\
\text { Student accomplishes the } \\
\text { following: } \\
\text { perspectives pertaining to the } \\
\text { problem may identify } \\
\text { perspectives not discussed } \\
\text { explicitly in case }\end{array}$ & $\begin{array}{l}\text { - Assembles an analysis of } \\
\text { multiple perspectives } \\
\text { pertaining to the problem } \\
\text { Student accomplishes } \\
\text { previous and one or more of } \\
\text { the following: } \\
\quad \text { explains the influences } \\
\text { on differing perspectives } \\
\text { compares and/or } \\
\text { contrasts differing } \\
\text { perspectives }\end{array}$ & $\begin{array}{l}\text { - Integrates multiple } \\
\text { perspectives into a } \\
\text { multifaceted } \\
\text { interpretation of the } \\
\text { problem and/or } \\
\text { solution(s) } \\
\text { Student accomplishes } \\
\text { previous and one or more of } \\
\text { the following: } \\
\checkmark \quad \text { presents a } \\
\text { synthesized discussion of } \\
\text { multiple perspectives } \\
\checkmark \quad \text { offers possible } \\
\text { solution(s) for the problem } \\
\checkmark \quad \text { evaluates the } \\
\text { problem and/or solution(s) }\end{array}$ \\
\hline
\end{tabular}

(9) Florida International University, 2012 


\section{APPENDIX H}

Recruitment email announcement 
Hello Faculty member,

My name is Eduardo Hernandez. I am a doctoral student in the College of Education Department of Teaching and Learning. For my dissertation, I am conducting a study exploring if and how university faculty members who have attended global learning professional development workshops perceive global learning as transformational learning. I am conducting a survey of university faculty members who have attended global learning professional development workshops in order to explore if and how university faculty members perceive global learning as transformational learning. Your name came to my attention as a possible participant in this survey because, according to the Office of Global Learning Initiatives, you have participated in a global learning professional development workshop.

This IRB approved survey can be completed in approximately 10 minutes. All responses to this survey will be kept private and will be protected to the fullest extent provided by law. Confidentiality will be given to survey responses through the assignment of a code by the Qualtrics software used to administer the survey.

If you would like to participate in my study or learn more about this study, simply click on the hyperlink below to do so. Your Anonymous Survey Link:

\section{Click here to learn more about the survey}


The feedback received from this survey can give FIU and the larger research community important knowledge of how faculty members view global learning.

Thank you in advance for your willingness to participate in this study.

Eduardo Hernandez

Doctoral Candidate, Department of Curriculum and Instruction

College of Education

Florida International University 
APPENDIX I

Online survey consent form 


\begin{tabular}{|l|l|}
\hline FIU IRB & $12 / 11 / 2014$ \\
\hline FIU IRB & $12 / 11 / 2015$ \\
\hline FIU IRB & IRB-14-0382 \\
\hline
\end{tabular}

Please read the following information before deciding to consent to participate in the study.

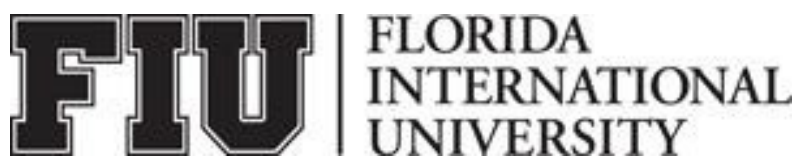

\section{ADULT ONLINE CONSENT TO PARTICIPATE IN A RESEARCH STUDY}

Exploring How Transformational Experiences of

Faculty in Global Learning Workshops Inform Practice

\section{PURPOSE OF THE STUDY}

The purpose of this dissertation is to add to the literature on

global learning, professional development, and transformational learning.

\section{DURATION OF THE STUDY}

It is estimated that survey completion will take five to 15 minutes

of your time. If you choose to do a follow up interview, it is estimated 
that the follow up interview could take approximately 45 minutes of your time.

\section{PROCEDURES}

The participant will respond to survey questions in the Qualtrics

program. Survey questions on transformational learning are made up of close-ended questions that can only be answered with either a "yes" or "no" response. Several short answer open-ended questions on transformational learning are also included in the survey. This is followed by multiple choice and short answer questions related to the demographics of the participant. The follow up interview is made up of several open-ended questions and prompts.

\section{RISKS AND/OR DISCOMFORTS}

Participants can reasonably expect the minimal risks, harms, and discomforts associated with everyday life. The risk of discomfort with a question is minimal and unlikely. The participant can choose not to answer the question or stop participating in the study at any time. There is a minimal and unlikely risk to confidentiality and anonymity. Each participant will be given a code or pseudonym to maintain their anonymity. Anything a participant may say, write, or type that can 


\begin{tabular}{|l|l|}
\hline FIU IRB Approval: & $12 / 11 / 2014$ \\
\hline FIU IRB Expiration: & $12 / 11 / 2015$ \\
\hline FIU IRB Number: & IRB-14-0382 \\
\hline
\end{tabular}

identify them or link them to the study will be removed from the data.

\section{BENEFITS}

There are no known benefits that may be associated with your participation in this study.

\section{CONFIDENTIALITY}

The records of this study will be kept private and will be protected to the fullest extent provided by law. Confidentiality will be given to survey responses through the assignment of a code by the Qualtrics software used to administer the survey. Survey data will remain anonymous unless the participant consents to a follow up interview. In that case, interview data will be combined with survey data and the researcher and his/ her research committee will be able to link a participant to their responses. The researcher will use the Qualtrics code and/or a pseudonym to make the identity of the participant anonymous to other people when publishing the data in any form.

\section{COMPENSATION \& COSTS}

You will not receive any payment or reimbursement for your 
participation. You will not be responsible for any costs to participate in this study.

\section{RIGHT TO DECLINE OR WITHDRAW}

Your participation in this study is voluntary. You are free to participate in the study or withdraw your consent at any time during the study. Your withdrawal or lack of participation will not affect any benefits to which you are otherwise entitled.

\section{RESEARCHER CONTACT INFORMATION}

If you have any questions about the purpose, procedures, or any other issues relating to this research study you may contact Dr. Hilary C. Landorf at Florida International University, 11200 SW 8th St, Miami Fl. 33199, phone number 305 xxx-xxxx, xxxxxxx@fiu.edu or Eduardo Hernandez at Florida International University, 11200 SW

8th St, Miami Fl. 33199, phone number 305 xxx-xxxx, xxxxx@fiu.edu. IRB CONTACT INFORMATION

If you would like to talk with someone about your rights of being a subject in this research study or about ethical issues with this research study, you may contact the FIU Office of Research Integrity by phone at 305-348-2494 or by email at ori@fiu.edu. 


\section{PARTICIPANT}

\begin{tabular}{|l|l|}
\hline FIU IRB Approval: & $12 / 11 / 2014$ \\
\hline FIU IRB Expiration: & $12 / 11 / 2015$ \\
\hline FIU IRB Number: & IRB-14-0382 \\
\hline
\end{tabular}

\section{AGREEMENT}

I have read the information in this consent form and agree to participate in this study. I have had a chance to ask any questions I have about this study, and they have been answered for me. By clicking on the "consent to participate" button below I am providing my informed consent.

(If you wish to be linked to this study through a hardcopy of a written adult consent form you can sign, please contact the researcher).

Consent to participate 


\section{APPENDIX J}

Interview consent form 


\begin{tabular}{|l|l|}
\hline FIU IRB Approval: & $12 / 11 / 2014$ \\
\hline FIU IRB Expiration: & $12 / 11 / 2015$ \\
\hline FIU IRB Number: & IRB-14-0382 \\
\hline
\end{tabular}

Please read the following information before deciding to consent to participate in the study.

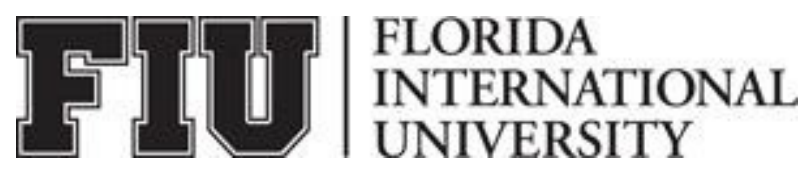

\section{ADULT ONLINE CONSENT TO PARTICIPATE IN A RESEARCH STUDY}

Exploring How Transformational Experiences of

Faculty in Global Learning

Workshops Inform Practice

\section{PURPOSE OF THE STUDY}

The purpose of this dissertation is to add to the literature on

global learning, professional development, and transformational

learning. 


\begin{tabular}{|l|l|}
\hline FIU IRB & $12 / 11 / 2014$ \\
\hline FIU IRB & $12 / 11 / 2015$ \\
\hline FIU IRB & IRB-14-0382 \\
\hline
\end{tabular}

\section{DURATION OF THE STUDY}

If you choose to do a follow up interview, it is estimated that the follow up interview could take approximately 45 minutes of your time.

\section{PROCEDURES}

The follow up interview is made up of several open-ended questions and prompts.

\section{RISKS AND/OR DISCOMFORTS}

Participants can reasonably expect the minimal risks, harms, and discomforts associated with everyday life. The risk of discomfort with a question is minimal and unlikely. The participant can choose not to answer the question or stop participating in the study at any time. There is a minimal and unlikely risk to confidentiality and anonymity. Each participant will be given a code or pseudonym to maintain their anonymity. Anything a 


\begin{tabular}{|l|l|}
\hline FIU IRB Approval: & $12 / 11 / 2014$ \\
\hline FIU IRB Expiration: & $12 / 11 / 2015$ \\
\hline FIU IRB Number: & IRB-14-0382 \\
\hline
\end{tabular}

participant may say, write, or type that can identify them or link

them to the study will be removed from the data.

\section{BENEFITS}

There are no known benefits that may be associated with your participation in this study.

\section{CONFIDENTIALITY}

The records of this study will be kept private and will be protected to the fullest extent provided by law. The researcher will use the Qualtrics code and/or a pseudonym to make the identity of the participant anonymous to other people when publishing the data in any form. The audio recordings of the interviews will be stored in encrypted files in an encrypted drives and laptops secured in a locked cabinet in the researcher's office. All recordings will be destroyed after three years from the completion of the study.

\section{COMPENSATION \& COSTS}

You will not receive any payment or reimbursement for your participation. You will not be responsible for any costs to participate in this study. 


\section{RIGHT TO DECLINE OR WITHDRAW}

Your participation in this study is voluntary. You are free to participate in the study or withdraw your consent at any time during the study. Your withdrawal or lack of participation will not affect any benefits to which you are otherwise entitled.

\section{RESEARCHER CONTACT INFORMATION}

If you have any questions about the purpose, procedures, or any other issues relating to this research study you may contact Dr. Hilary C. Landorf at Florida International University, 11200 SW 8th St, Miami Fl. 33199, phone number 305 xxx-xxxx, xxxxxxx@fiu.edu or Eduardo Hernandez at Florida International University, 11200 SW

8th St, Miami Fl. 33199, phone number 305 xxx-xxxx, xxxxxx@fiu.edu. IRB CONTACT INFORMATION

If you would like to talk with someone about your rights of being a subject in this research study or about ethical issues with this research study, you may contact the FIU Office of Research Integrity by phone at 305-348-2494 or by email at ori@fiu.edu. 


\begin{tabular}{|l|c|}
\hline FIU IRB Approval: & $12 / 11 / 2014$ \\
\hline FIU IRB Expiration: & $12 / 11 / 2015$ \\
\hline FIU IRB Number: & IRB-14-0382 \\
\hline
\end{tabular}

\section{PARTICIPANT AGREEMENT}

I have read the information in this consent form and agree to participate in this study. I have had a chance to ask any questions I have about this study, and they have been answered for me. I understand that I will be given a copy of this form for my records.

Signature of Participant

Printed Name of Participant
Date

\section{Date}




\section{APPENDIX K}

Overview of demographic data from surveys labeled Non-Transformational

Learning 


\section{NON-TRANSFORMATIONAL LEARNING SURVEYS}

School or college of faculty member, the number of GL courses they have taught, how often, and for how long.

\begin{tabular}{|c|c|c|c|c|c|c|c|}
\hline $\begin{array}{l}\text { School or } \\
\text { College }\end{array}$ & Response & $\begin{array}{l}\text { Number } \\
\text { of GL } \\
\text { courses } \\
\text { taught }\end{array}$ & Response & $\begin{array}{l}\text { How } \\
\text { often a } \\
\text { GL } \\
\text { course is } \\
\text { taught }\end{array}$ & Response & $\begin{array}{l}\text { How long } \\
\text { faculty } \\
\text { member } \\
\text { has taught } \\
\text { a GL } \\
\text { course }\end{array}$ & Response \\
\hline $\begin{array}{l}\text { College of } \\
\text { Architecture } \\
\text { and Arts }\end{array}$ & 1 & None & 3 & $\begin{array}{l}\text { Every } \\
\text { semester }\end{array}$ & 4 & Never & 3 \\
\hline $\begin{array}{l}\text { College of Arts } \\
\text { and Sciences }\end{array}$ & 6 & $\begin{array}{l}\text { One } \\
\text { course }\end{array}$ & 6 & $\begin{array}{l}\text { One } \\
\text { semester } \\
\text { a year }\end{array}$ & 1 & $\begin{array}{l}\text { Not } \\
\text { Applicable }\end{array}$ & 2 \\
\hline $\begin{array}{l}\text { College of } \\
\text { Business }\end{array}$ & 0 & $\begin{array}{l}\text { Two } \\
\text { courses }\end{array}$ & 1 & $\begin{array}{l}\text { Two } \\
\text { semesters } \\
\text { in a year }\end{array}$ & 2 & $\begin{array}{l}\text { Beginning } \\
\text { of global } \\
\text { learning } \\
\text { initiative }\end{array}$ & 1 \\
\hline $\begin{array}{l}\text { College of } \\
\text { Education }\end{array}$ & 0 & $\begin{array}{l}\text { Three } \\
\text { courses }\end{array}$ & & $\begin{array}{l}\text { Every } \\
\text { semester } \\
\text { in the } \\
\text { past }\end{array}$ & 0 & $\begin{array}{l}\text { One year } \\
\text { or less }\end{array}$ & 2 \\
\hline $\begin{array}{l}\text { College of } \\
\text { Engineering } \\
\text { and Computing }\end{array}$ & 1 & $\begin{array}{l}\text { Four } \\
\text { courses }\end{array}$ & 0 & $\begin{array}{l}\text { Every } \\
\text { few years }\end{array}$ & 0 & Two years & 1 \\
\hline Honors College & 2 & $\begin{array}{l}\text { Five } \\
\text { courses }\end{array}$ & 0 & Never & 3 & $\begin{array}{l}\text { Three } \\
\text { years }\end{array}$ & 0 \\
\hline College of Law & 0 & $\begin{array}{l}\text { One or } \\
\text { more in } \\
\text { the past }\end{array}$ & 0 & $\begin{array}{l}\text { Not } \\
\text { applicabl } \\
\text { e }\end{array}$ & 0 & Four years & 3 \\
\hline Herbert & 0 & Any & 1 & All & 1 & Five years & 0 \\
\hline Wertheim & & & & & & & \\
\hline $\begin{array}{l}\text { College of } \\
\text { Medicine }\end{array}$ & & & & & & & \\
\hline $\begin{array}{l}\text { Nicole } \\
\text { Wertheim }\end{array}$ & 1 & $\begin{array}{l}\text { Not } \\
\text { applicabl } \\
\text { e }\end{array}$ & 1 & & & $\begin{array}{l}\text { Six to } \\
\text { eight years } \\
\text { or more }\end{array}$ & 2 \\
\hline
\end{tabular}


Nursing and

Health

Sciences

Chaplin 0

School of

Hospitality

and Tourism

Management

School of 0

Journalism

and Mass

Communication

$\mathrm{S}$

Other: $\quad 1$

Total 
Race and Ethnicity of faculty members as reported in surveys categorized as Non-Transformational Learning.

\begin{tabular}{|c|c|c|c|}
\hline Ethnicity & Responses & Race & Responses \\
\hline African & 0 & $\begin{array}{l}\text { White (Non- } \\
\text { Hispanic) }\end{array}$ & 6 \\
\hline African American & 0 & Black & 0 \\
\hline Asian & 2 & Hispanic & 1 \\
\hline \multirow[t]{3}{*}{ Asian American } & 0 & Asian or & 2 \\
\hline & & Pacific & \\
\hline & & Islander & \\
\hline \multirow[t]{2}{*}{ Caucasian } & 1 & Other (Please & 3 \\
\hline & & specify) & \\
\hline Caribbean & 0 & & \\
\hline European & 1 & & \\
\hline European American & 0 & & \\
\hline Hispanic & 1 & & \\
\hline Jewish & 0 & & \\
\hline Latin Hispanic & 0 & & \\
\hline Middle Eastern & 0 & & \\
\hline Mixed & 1 & & \\
\hline White & 2 & & \\
\hline White European & 1 & & \\
\hline White Hispanic & 0 & & \\
\hline White Latino & 0 & & \\
\hline White Non Hispanic & 1 & & \\
\hline Chose not to answer & 4 & & \\
\hline Total & & & 12 \\
\hline
\end{tabular}




\section{APPENDIX L}

Overview of demographic data from surveys labeled Partial Learning 


\section{PARTIAL LEARNING SURVEYS}

School or college of faculty member, the number of GL courses they have taught, how often, and for how long.

\begin{tabular}{|c|c|c|c|c|c|c|c|}
\hline $\begin{array}{l}\text { School or } \\
\text { College }\end{array}$ & Response & $\begin{array}{l}\text { The } \\
\text { number } \\
\text { of GL } \\
\text { courses } \\
\text { taught }\end{array}$ & Response & $\begin{array}{l}\text { How } \\
\text { often GL } \\
\text { courses } \\
\text { are taught }\end{array}$ & Response & $\begin{array}{l}\text { How long } \\
\text { faculty } \\
\text { member } \\
\text { has taught } \\
\text { a GL } \\
\text { course }\end{array}$ & Response \\
\hline $\begin{array}{l}\text { College of } \\
\text { Architecture } \\
\text { and Arts }\end{array}$ & 0 & None & 1 & $\begin{array}{l}\text { Every } \\
\text { semester }\end{array}$ & 11 & Never & 2 \\
\hline $\begin{array}{l}\text { College of Arts } \\
\text { and Sciences }\end{array}$ & 12 & $\begin{array}{l}\text { One } \\
\text { course }\end{array}$ & 15 & $\begin{array}{l}\text { One } \\
\text { semester } \\
\text { a year }\end{array}$ & 4 & $\begin{array}{l}\text { Not } \\
\text { Applicable }\end{array}$ & 0 \\
\hline $\begin{array}{l}\text { College of } \\
\text { Business }\end{array}$ & 0 & $\begin{array}{l}\text { Two } \\
\text { courses }\end{array}$ & 2 & $\begin{array}{l}\text { Two } \\
\text { semesters } \\
\text { in a year }\end{array}$ & 5 & $\begin{array}{l}\text { Beginning } \\
\text { of global } \\
\text { learning } \\
\text { initiative }\end{array}$ & 0 \\
\hline $\begin{array}{l}\text { College of } \\
\text { Education }\end{array}$ & 3 & $\begin{array}{l}\text { Three } \\
\text { courses }\end{array}$ & 2 & $\begin{array}{l}\text { Every } \\
\text { semester } \\
\text { in the } \\
\text { past }\end{array}$ & 0 & $\begin{array}{l}\text { One year } \\
\text { or less }\end{array}$ & 5 \\
\hline $\begin{array}{l}\text { College of } \\
\text { Engineering and } \\
\text { Computing }\end{array}$ & 2 & $\begin{array}{l}\text { Four } \\
\text { courses }\end{array}$ & 0 & $\begin{array}{l}\text { Every } \\
\text { few years }\end{array}$ & 1 & Two years & 3 \\
\hline Honors College & 1 & $\begin{array}{l}\text { Five } \\
\text { courses }\end{array}$ & 1 & Never & 1 & $\begin{array}{l}\text { Three } \\
\text { years }\end{array}$ & 9 \\
\hline College of Law & 0 & One or & 3 & Not & 2 & Four years & 1 \\
\hline
\end{tabular}




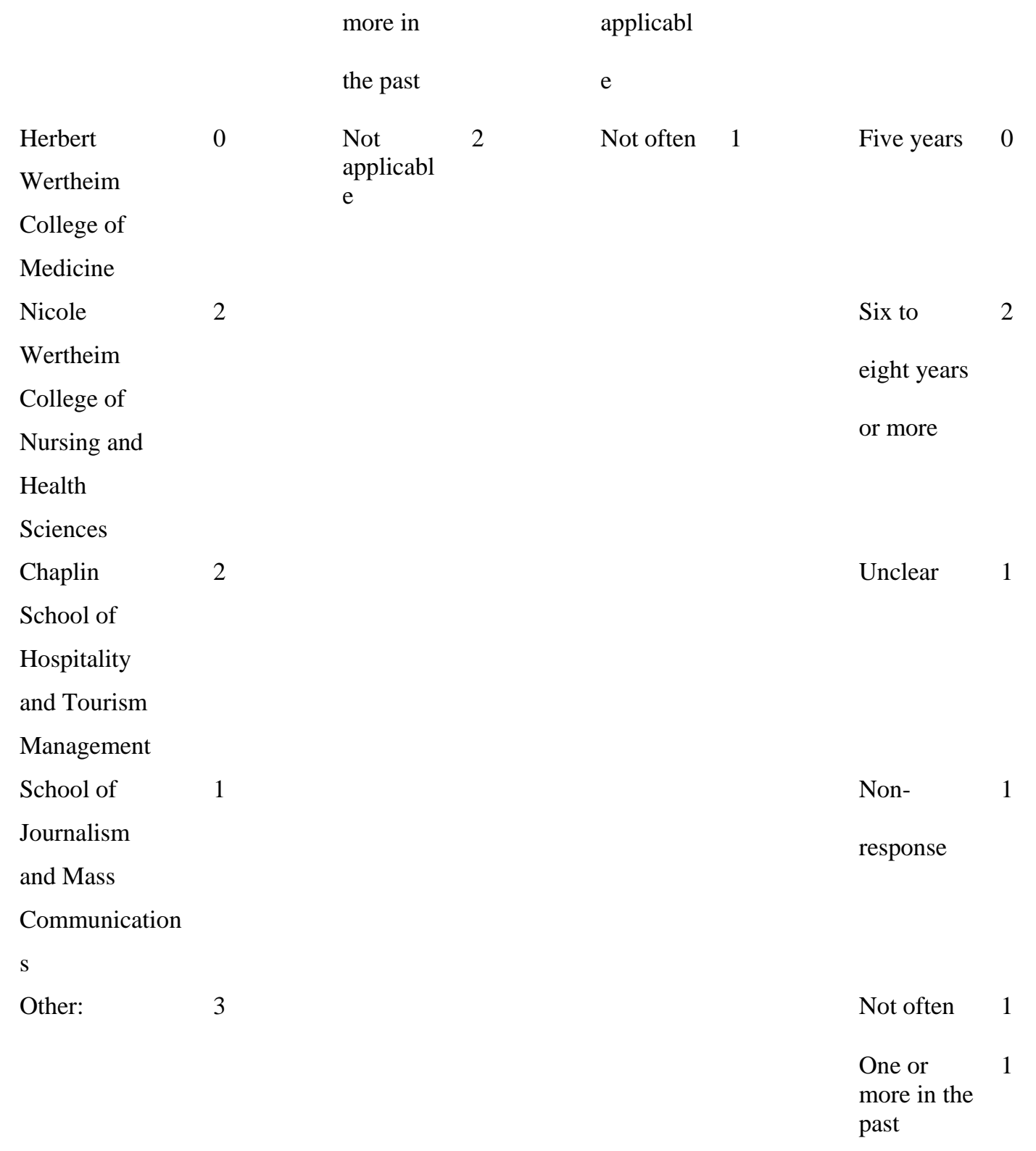

Total

26

26

26

PARTIAL LEARNING SURVEYS 
Ethnicity and race of faculty members as reported in surveys categorized as partial learning.

\begin{tabular}{|c|c|c|c|}
\hline Ethnicity & Responses & Race & Responses \\
\hline African & 0 & $\begin{array}{l}\text { White (Non- } \\
\text { Hispanic) }\end{array}$ & 15 \\
\hline African American & 1 & Black & 2 \\
\hline American & 1 & Hispanic & 4 \\
\hline \multirow{3}{*}{ Asian } & 1 & Asian or & 1 \\
\hline & & Pacific & \\
\hline & & Islander & \\
\hline & 1 & Other (Please & 4 \\
\hline Asian American & & specify) & \\
\hline Caucasian & 3 & & \\
\hline Caribbean & 1 & & \\
\hline \multicolumn{4}{|l|}{ European } \\
\hline European American & 0 & & \\
\hline Hispanic & 3 & & \\
\hline Jewish & 0 & & \\
\hline Latin Hispanic & 0 & & \\
\hline Middle Eastern & 0 & & \\
\hline Mixed & 2 & & \\
\hline White & 4 & & \\
\hline White European & 0 & & \\
\hline White Hispanic & 1 & & \\
\hline
\end{tabular}


White Latino 1

White Non Hispanic 5

Chose not to answer 3

Total

26 
VITA

\section{EDUARDO HERNANDEZ}

2004 Bachelor of Arts in History

Florida International University

Miami FL., USA.

2008 Masters of Science in Curriculum and Instruction in

Social Studies Education.

Florida International University

Miami, FL., USA.

2009 Masters of Library and Information Science

University of South Florida

Tampa, FL. USA.

2011 Education Specialist degree in Curriculum and Instruction in Social Studies Education.

Florida international University

Miami, Fl., USA.

2015 Doctorate of Education in Curriculum and Instruction in

Social Studies Education.

Florida International University.

Miami, Fl., USA.

\section{PRESENTATIONS AND PAPERS}

Hernandez, E. (2013). Telling stories: Fighting Latinalo nihilism in social studies classrooms. Paper presented at the Annual Meeting of the National Council of the Social Studies. St. Louis: NCSS.

Hernandez, E. (2013). Teaching narrative, Trayvon Martin, and lasting international movements. Paper presented at the South East Regional Conference of the Comparative and International Education Symposium. Tallahassee: Florida State University.

Hernandez, E. (2013). Theorizing strategic intersectionalities of female African dropouts through autoethnographic education. Paper presented at the South East Regional Conference of the Comparative and International Education Symposium. Tallahassee: Florida State University. 
Hernandez, E. (2013). Testimonios para justicia: Using LatCrit, autoethnography, and counterstory to teach about and resist Latino nihilism in the K-12 classroom.

In M. S. Plakhotnik, T. Lucas, \& J. Pena (Eds.), Proceedings of the 12th Annual South Florida Education Research Conference. (pp.102-109) Miami: Florida International University. http://coeweb.fiu.edu/research_conference/

Hernandez, E. (2013). Life, social studies, and the pursuit of happiness: Using classroom-based multicultural democratic education to challenge conservative notions of civic education. In M. S. Plakhotnik, T. Lucas, \& J. Pena (Eds.), Proceedings of the 12th Annual South Florida Education Research Conference. (pp.78-85) Miami: Florida International University. http://coeweb.fiu.edu/research_conference/

Hernandez, E. (2013). Promoting an Operation Cease Fire approach in Miami Dade classrooms in order to prevent youth crime gun violence in the community. In M. S. Plakhotnik, T. Lucas, \& J. Pena (Eds.), Proceedings of the 12th Annual South Florida Education Research Conference (pp.86-93). Miami: Florida International University. http://coeweb.fiu.edu/research_conference/

Hernandez, E. (2013). The framing of the aftermath of the Chicago teacher's strike of 2012 in the media: Its implications for teachers as public intellectuals. In M. S. Plakhotnik, T. Lucas, \& J. Pena (Eds.), Proceedings of the 12th Annual South Florida Education Research Conference (pp.94-101). Miami: Florida International University. http://coeweb.fiu.edu/research_conference/

Hernandez, E. (2013). Promoting cosmopolitanism in global learning to support democracy through humanization in global citizenship. Paper presented at the $57^{\text {th }}$ annual conference of the Comparative and International Education symposium. New Orleans: CIES.

Hernandez, E. (2013). Social science research through human terrain systems as a form of Foucauldian surveillance. Paper presented at the $57^{\text {th }}$ annual conference of the Comparative and International Education symposium. New Orleans: CIES.

Hernandez, E. (2012). A phenomenological study of undergraduate students perceptions of global learning curriculum. Paper presented in the New Scholars workshop in the $56^{\text {th }}$ annual conference of the Comparative and International Education Symposium. San Juan: CIES.

Hernandez, E. (2012). Rural Chinese homosexual men and the biopolitical implications of AIDS education campaigns in rural China. Paper presented at the Asia Special Interest Group in the $56^{\text {th }}$ annual conference of the Comparative and International Education Symposium. San Juan: CIES. 